\title{
Examining the Impact of a Short-Term Psychological Skills Training Program on Dancers' Coping Skills, Pain Appraisals, and Injuries
}

\author{
Leigh A. Bryant
}

Follow this and additional works at: https://researchrepository.wvu.edu/etd

\section{Recommended Citation}

Bryant, Leigh A., "Examining the Impact of a Short-Term Psychological Skills Training Program on Dancers' Coping Skills, Pain Appraisals, and Injuries" (2017). Graduate Theses, Dissertations, and Problem Reports. 5277.

https://researchrepository.wvu.edu/etd/5277

This Dissertation is protected by copyright and/or related rights. It has been brought to you by the The Research Repository @ WVU with permission from the rights-holder(s). You are free to use this Dissertation in any way that is permitted by the copyright and related rights legislation that applies to your use. For other uses you must obtain permission from the rights-holder(s) directly, unless additional rights are indicated by a Creative Commons license in the record and/ or on the work itself. This Dissertation has been accepted for inclusion in WVU Graduate Theses, Dissertations, and Problem Reports collection by an authorized administrator of The Research Repository @ WVU.

For more information, please contact researchrepository@mail.wvu.edu. 
Examining the Impact of a Short-Term Psychological Skills Training Program on Dancers' Coping Skills, Pain Appraisals, and Injuries

Leigh A. Bryant, MS, MA

\author{
Dissertation submitted to \\ the College of Physical Activity and Sport Sciences \\ at West Virginia University \\ in partial fulfillment of the requirements for the degree of \\ Doctorate of Philosophy in \\ Sport and Exercise Psychology
Damien Clement, PhD, ATC, Chair
Edward F. Etzel, EdD
Kelly Knox, MFA
Monica Leppma, $\mathrm{PhD}$
Sam Zizzi, EdD \\ College of Physical Activity and Sport Sciences
}

Morgantown, West Virginia

2017

Keywords: intervention, injury, psychological skills training, dance, pain appraisal, coping Copyright 2017 Leigh A. Bryant 


\begin{abstract}
Examining the Impact of a Short-Term Psychological Skills Training Program on Dancers' Coping Skills, Pain Appraisals, and Injuries
\end{abstract}

Leigh A. Bryant

Psychological skills interventions are often conducted with individual athletes and sports teams in an effort to build mental toughness, prevent injury, or enhance performance. Dancers remain an underserved population in the delivery of sport/performance psychology services, with extremely limited literature addressing college dance students. The dance community has several inherent physical and psychosocial demands, which can promote resilience. However, dancers may also strive to meet these demands by employing unhealthy coping strategies that could be linked to maladaptive appraisals and increased injury risk. The present study examined the impact of a psychological skills intervention program on college dance students' $(N=30)$ coping skills, pain appraisals, and injuries over a six-week period. A two-group pre-test/post-test quasiexperimental methodology was used to capture the potential influence of the intervention program. At the end of the six-week period, there were no significant differences observed between the treatment and control groups on the three major constructs. In particular, the results demonstrated the need for a single, operational definition of injury within the dance context. A focus group discussion led to several recommendations for future research and improvements for mental skills and life skills intervention programs. Best practices for scholars, applied practitioners, and dance educators are also discussed. 


\section{ACKNOWLEDGEMENTS}

I would like to thank my Committee Chair, Dr. Damien Clement, for your thorough feedback and encouragement throughout the dissertation process.

I would also like to thank the other members of my committee-Dr. Ed Etzel, Dr. Monica Leppma, Professor Kelly Knox, and Dr. Sam Zizzi - for the time and energy that you put into helping me complete this project.

A big "thanks" to all of the faculty, staff, and students in the Sport \& Exercise Psychology and Counseling programs at West Virginia University.

Another big "thanks" to the dancers who took part in this study, and the instructors and administrators who supported their participation.

Special thanks to Alison Coates, Jana Fogaça, Stefanee Maurice, and Anna Onderik for your interest in and contributions to this project.

Finally, thank you to my parents - Neil and Jennifer Bryant — and to my husband — Kurt Skvarla - for your love and support. 


\section{TABLE OF CONTENTS}

$\underline{\text { Page }}$

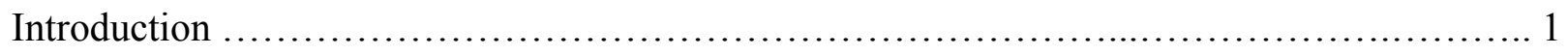

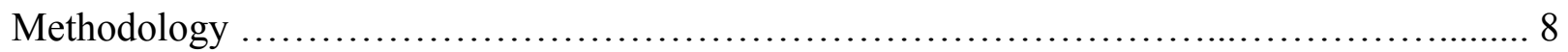

Research Design ..................................................................... 8

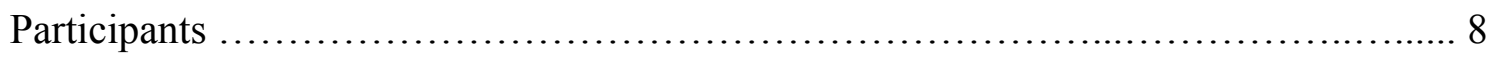

Dance Program Characteristics and Setting ........................................ 9

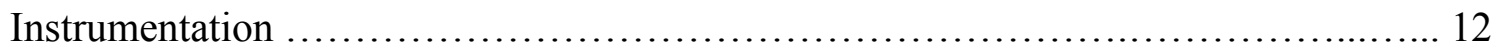

Basic Demographics Survey .................................................... 12

Athletic Coping Skills Inventory-28 …......................................... 12

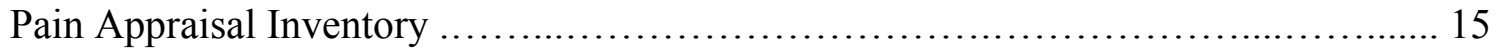

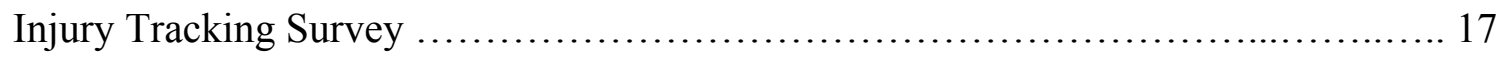

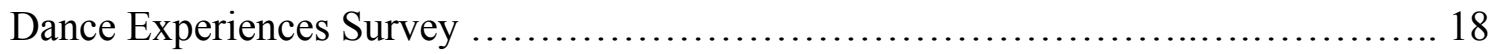

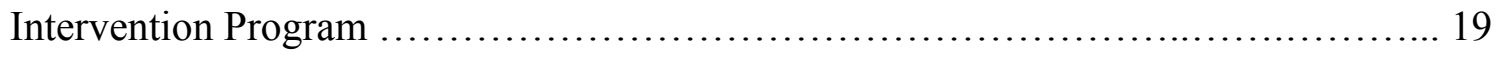

Adherence Journal .......................................................... 21

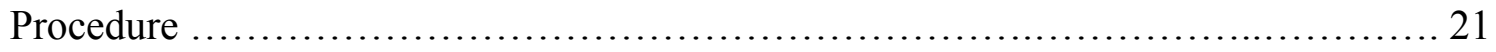

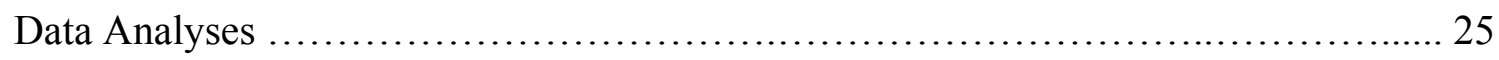

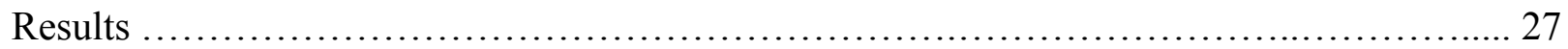

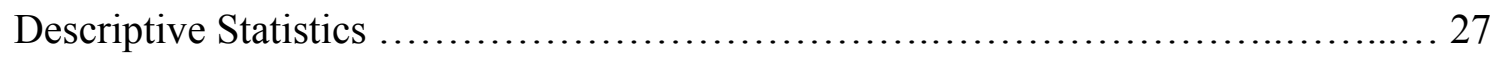

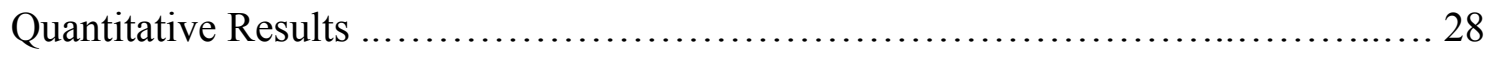

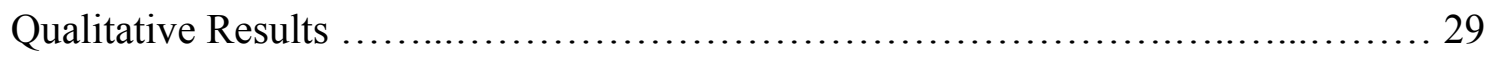

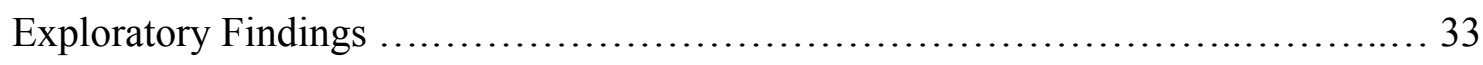




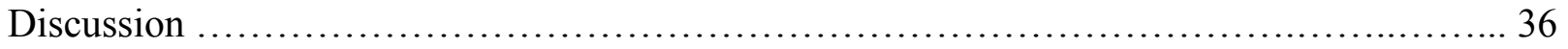

Limitations .......................................................................... 53

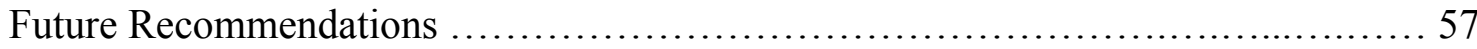

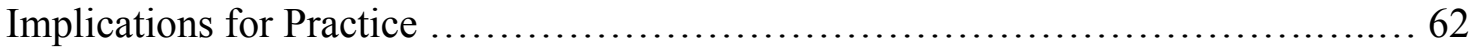

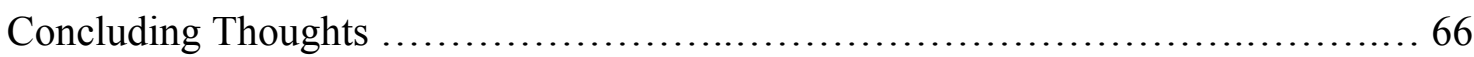

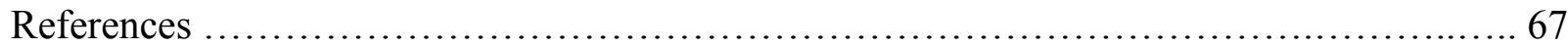

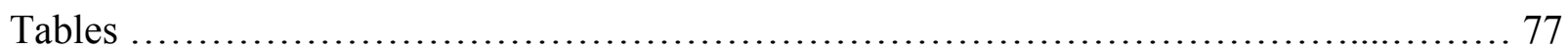

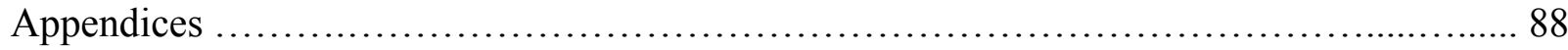

Appendix A: Extended Review of Literature .................................. 88

Appendix B: Basic Demographics Survey ....................................138

Appendix C: Athletic Coping Skills Inventory-28 ….......................... 141

Appendix D: Pain Appraisal Inventory ........................................ 144

Appendix E: Injury Tracking Survey ...................................... 146

Appendix F: Dance Experiences Survey ...................................... 149

Appendix G: Intervention Protocol Outline …................................ 155

Appendix H: Intervention Program Exercises and Activities ...................... 157

Appendix I: Adherence Journal ............................................ 167

Appendix J: Participant Contact Information Form................................ 168

Appendix K: Semi-Structured Interview Guide for Focus Group Discussion ........ 169

Appendix L: Stakeholder Interview Findings ............................... 170

Appendix M: Sample Self-Talk Statements and Associated Bible Verses ............ 173 


\section{Introduction}

Dancers are considered to be both artists and athletes, given the rigor of their physical training, the necessity of self-expression, and the emphasis on the mind-body connection (Dick et al., 2013). A dancer's body is trained to move in complex, highly-technical ways and to stand in atypical positions (e.g., on the tips of one's toes). For this reason, dancers often have timelimited careers, with very few individuals performing beyond their thirties (Kelman, 2000; Turner \& Wainwright, 2003; Watson, 2013). Additionally, the path to artistic perfection is a never-ending process, because one's performance cannot be objectively measured by a judge or audience member (Dick et al., 2013; Estanol, Shepherd, \& MacDonald, 2013). Many dancers spend the entirety of their careers making technical and aesthetic improvements. They strive to maintain a slim and athletic physique, endure long rehearsal hours, and execute advanced technical requirements (Grove, Main, \& Sharp, 2013; Hamilton \& Robson, 2006; Shah, 2008).

With no final score to use as a measure of success, dancers often need to engage in self-reflection to assess their strengths and areas for growth.

As Kelman (2000) has stated, “dancers must be physically strong and have a high tolerance for pain. The performing artist's instrument is their body" (p. 431). The mentality of "no pain, no gain" that is often used to characterize an intense sport climate may also be an appropriate description of a passionate dancer's assessment of pain or injury (Anderson \& Hanrahan, 2008). When adopted and reinforced, this "push through it" mindset can be one that is self-motivating and temporarily adaptive. Nevertheless, it is also possible that this mindset can contribute to injury risk if the pain is not properly identified and addressed (Anderson \& Hanrahan, 2008; Rip, Fortin, \& Vallerand, 2006). In other words, dancers who conceal or ignore physical pain in order to keep training and performing (Krasnow, 2005; Noh \& Morris, 2004) 
may be doing more damage to their body, and negatively influencing the environment in which they want to thrive (Anderson \& Hanrahan, 2008).

The physical demands and the socio-cultural climate of many schools and studios (Dick et al., 2013; Grove, Main, \& Sharp, 2013) may lead dancers to a metaphorical tipping point. In some cases, dancers demonstrate resilience in the face of such pressures and engage in strategies that can enhance performance and well-being (e.g., Rivers, 2014). However, a lack of coping skills can lead to disruptions in dancers' ambitions, or the adoption of destructive behaviors (Kelman, 2000; van Staden, Myburgh, \& Poggenpoel, 2009). For instance, obsessive passion can lead to inattentiveness to pain and injury (Rip, Fortin, \& Vallerand, 2006). Similarly, low levels of self-awareness about how to properly manage pain or recognize injuries that require medical attention might put dancers' short- and long-term health at risk (Nordin-Bates et al., 2011).

Therefore, it is critical to understand dancers' appraisals of pain, how these appraisals relate to the desire to keep improving, and what factors influence dancers who engage in positive or adaptive coping strategies, such as those that might be introduced in a prevention or intervention program. As stated by Noh, Morris, and Andersen (2007), "the focus on enhancing coping skills...[may] help dancers cope more effectively with the demands of the dance environment and reduce injury incidence" (p. 28). To date, very little research has examined the delivery of psychological skills training programs to dancers (Noh, Morris, \& Andersen, 2007). Historically, these programs have focused on repairing — rather than preventing — dysfunctional coping strategies, unhelpful cognitive habits, and injuries (Hays, 2002).

The literature shows that in an eight-month period, up to $97 \%$ of university dance majors may experience an injury (Kerr, Krasnow, \& Mainwaring, 1992). Other researchers have also reported high rates of injury, with one sample of pre-professional dancers sustaining an average 
of 1.42 injuries per dancer over the course of a year (Ekegren et al., 2013). Dancers deal with both acute and chronic injuries such as ankle sprains, anterior cruciate ligament tears, tendonitis, and stress fractures (Bauman, Gallagher, \& Hamilton, 1996; Dick et al., 2013). As such, they are likely to cope with injury related pain in addition to generalized pain that may stem from training and performing.

Sustaining an injury may have several consequences for a dancer (Mainwaring, Krasnow, $\&$ Kerr, 2001). More specifically, being injured may necessitate taking time off from dancing, which may equate to missed opportunities to advance one's development. Dancers who have a strong athletic identity (Brewer, Van Raalte, \& Linder, 1993) and/or contingent self-worth (Hall \& Hill, 2012) may experience decreases in self-esteem should they become injured or have to manage chronic pain. Moreover, for dancers who are employed or seeking employment, the inability to work can result in financial strain (Kelman, 2000; Wainwright, Williams, \& Turner, 2005). Financial pressures could lead to additional stress, and possibly the need to pursue a different career.

These potential consequences may serve as motivation for dancers to keep injuries hidden. Dancers may self-medicate in an effort to treat or cover-up injuries and/or pain without interfering with regular training and performances (e.g., Wozny, 2016). While researchers have noted that some dancers do inform a teacher or director that something is bothering them, they will nevertheless continue to dance (Nordin-Bates et al., 2011). Nordin-Bates and colleagues (2011) have further suggested that dancers may view rest as a form of "non-constructive passivity" that could have consequences for their fitness, technical training, and/or status, as previously described (p. 82). Therefore, although missteps or improper technique can lead to physical injury in dance, sometimes injuries may occur due to one or more psychological and/or 
psychosocial factors (e.g., Noh \& Morris, 2004; Noh, Morris, \& Andersen, 2005; Nordin-Bates, Quested, Walker, \& Redding, 2012; Smith, Ptacek, \& Patterson, 2000).

Coping skills, pain appraisals, and injury status may be important to a dancer's mental well-being and performance-related outcomes. To characterize dancers' perceived stressors, Noh, Morris, and Andersen (2002) conducted a qualitative investigation with a sample of professional ballet dancers. The researchers found that fear of injury, dance directors' criticism, and competition for roles were prevalent. Additionally, over $60 \%$ of the dancers in their sample reported engaging in dysfunctional coping behaviors (e.g., drinking alcohol) as a means of dealing with perceived stress. Consistent with literature focused on stress and coping, researchers have identified several factors that may make individuals more susceptible to injury in a stressful situation. These factors include low levels of coping skills, high state-trait anxiety, and a history of several negative life events (e.g., Yatabe et al., 2014).

Consequently, it is important for dancers, and those who work with them, to be equipped with accurate, evidence-based knowledge about healthy coping skills, adaptive pain appraisals, and injury risk. Andersen and Williams (1988) have developed and revised a model that explores the relationship between stress and injury in sport (Williams \& Andersen, 1998). The model emphasizes personality, coping skills, and history of stressors as antecedents to injury risk (Andersen \& Williams, 1988; Noh, Morris, \& Andersen, 2005). Researchers have previously used this model with dancers to target coping skills as a protective factor for injury (e.g., Noh, Morris, \& Andersen, 2007).

In the present study, Williams and Andersen's model (1998) was used as a lens through which to view the role of interventions focused on coping skills, cognitive appraisals of pain, and injury risk in a collegiate environment. In particular, the impact that intervention content may 
have on cognitive appraisal was of interest to the primary researcher. While the model is often said to be linked to acute injury prediction, it was used in the present study to understand the relationship between intervention programming and cognitive appraisal of situations that could lead to or include dance-related pain and/or injury.

The model also highlights the potentially meaningful role of coping skills when managing perceived stress. Thus, learning skills to identify and manage stress has implications not only for dancers' performance-related outcomes, but also for their other life pursuits. A life skills approach to the intervention content may help dancers to better recognize the applicability of psychological skills training to their academics, social situations, and/or personal development (e.g., Danish, Petitpas, \& Hale, 1993). Accordingly, the primary researcher developed and selected instruments and program content tailored to a dance population, and, more specifically, for university-level dance students. The psychological skills training program was ultimately aimed at dancers' coping skills, appraisals of physical pain, and self-reported injuries over a sixweek time frame.

The present study sought to contribute to the current applied research literature in the field of sport/performance psychology. Noh and Morris (2004) previously used Williams and Andersen's (1998) model to design injury prevention programs with a sample of Korean dancers $(N=105)$. The authors examined the predictive power of coping skills, stress, social support, and anxiety on injury frequency and duration, further testing the veracity of the stress-injury model to the dance population. Building on this work, Noh, Morris, and Andersen (2007) published the findings of two intervention programs used with a sample of dancers $(N=35)$ for the purpose of injury prevention across a 24-week period. The first 12 weeks of the intervention program consisted of the delivery of coping skills content, and the subsequent 12 weeks were used to 
collect follow-up data as dancers practiced these skills. Their results suggested that teaching broad-based coping skills could in fact influence the duration of dancers' injuries. Noh and colleagues (2007) reported that dancers who were assigned to a treatment group spent less time injured than dancers who were assigned to a control group.

While some research focused on coping skills and injury has been conducted within the dance population, the current literature contains few intervention programs delivered to dancers in a group context. Consequently, the present study's methodology is rooted in literature from psychology, counseling, and family systems, which all, to some extent, utilize group sessions, cognitive-behavioral approaches, and brief interventions. Researchers have suggested that, in just a few sessions, individuals have the capacity to learn new material and make long-term behavioral changes (e.g., Bell, Skinner, \& Fisher, 2009; Meichenbaum, 1993). In this way, the present study allowed dancers to learn and then apply psychological skills into their training and performances.

It was hoped that these skills and strategies might positively influence dancers' stress management and injury susceptibility, and also translate to other life pursuits such as social situations or academics. Hamilton and Robson (2006) have stated that consultants who work with performing artists have the opportunity to teach "constructive strategies that enhance performance" while helping dancers to recognize and change self-destructive behaviors or coping methods (p. 257). The constructive strategies that they discussed included diaphragmatic breathing, relaxation exercises, positive self-talk, and imagery (Hamilton \& Robson, 2006).

These strategies have previously been taught to non-elite and collegiate athletes across a variety of sports, including gymnastics and rowing. For instance, over the course of a competition season, Kerr and Goss (1996) conducted individualized sessions with young adult 
gymnasts $(N=24)$ that focused on thought control, imagery, and self-talk. While no statistically significant findings were reported, the researchers found that the gymnasts who had participated in the intervention program had lower levels of negative athletic stress at the end of the season.

Furthermore, Perna, Antoni, Baum, Gordon, and Schneiderman (2003) conducted a threeweek intervention program that focused on stress management for college rowers $(N=34)$. Two practitioners taught athletes assigned to a treatment group a variety of skills for performance enhancement and personal development. These skills included deep breathing, progressive muscle relaxation, and imagery. At the conclusion of the intervention program, the researchers found that athletes who had been assigned to the treatment group had fewer reported injuries and illnesses compared to the athletes who were assigned to a control group. The results of these studies, in combination with their limitations and future recommendations, suggest that interventions focused on psychological skills and life skills development can have an important impact on young adult athletes with respect to performance and overall well-being.

Therefore, the purpose of the present study was to examine the impact of a short-term psychological skills training program on college dancers' self-reported coping skills, pain appraisal, and injuries. This psychological skills training program was rooted in the stress management and performance enhancement literature, and adopted a cognitive-behavioral approach. In accordance with the study's purpose, the four main research questions were: (1) Is there a difference in self-reported coping skills between dancers who participate in the intervention program and those who do not? (2) Is there a difference in self-reported pain appraisals between dancers who participate in the intervention program and those who do not? (3) Is the rate and severity of injuries affected by the intervention program? (4) What are the 
dancers' reactions to this intervention program, and, more specifically, what aspects of the program were useful and not useful, and why?

\section{Methodology}

\section{Research Design}

The present study utilized a two-group pre-test/post-test quasi-experimental design (Gay \& Airasian, 2003). Participants were assigned to a condition, as a group, based on the dance course in which they were enrolled for the spring 2016 semester. One class was assigned to be the treatment group; this group completed study-related instruments and received a short-term psychological skills intervention program on a weekly basis. The other class was assigned to be the control group; this group also completed study-related instruments without participating in the intervention program. This type of research design is commonly utilized in educational settings, where purely random assignment of participants to groups is not feasible or practical (Gay \& Airasian, 2003). The treatment group participants were currently enrolled in a modern dance course, and the control group participants were currently taking a dance conditioning course. These classes were part of the dance program curriculum and led by full-time faculty members at the institution.

\section{Participants}

Participants were male $(n=4)$ and female $(n=26)$ dance students $(N=30)$ studying at the same institution in the Mid-Atlantic region of the United States. Students were encouraged to participate by their instructors, but ultimately the decision to participate was their own. One student was deemed ineligible to participate due to age (less than 18 years), and one student in the control group asked to be removed from the study. 
Participants' ages ranged from 18 to 22 years $(M=19.77, S D=1.45)$. On average, dancers reported a range of 3 to 19 years of dance training $(M=13.30, S D=4.50)$. Participants represented all four class years, namely freshman or first year $(n=13)$, sophomore $(n=2)$, junior $(n=12)$, and senior $(n=3)$. Prior to their participation in this study, few dance students had experience working with a sport psychology consultant $(n=3)$. The remaining students $(n=27)$ indicated no prior experience with a sport psychology consultant in an individual or group setting. At the start of the study (i.e., during the first week), almost half of the participants ( $n=$ 13) indicated that they were currently suffering from one or more injuries; four injured dancers were in the control group, and the other nine were in the treatment group.

On average, dancers in the treatment group were 2.4 years older than dancers in the control group. Dancers in the control group were slightly younger $\left(M_{\text {age }}=18.60, S D=0.91\right)$ than dancers in the treatment group $\left(M_{\text {age }}=20.93, S D=0.80\right)$. Members of the control group reported a range of 14 to 25 hours of dance training per week. Members of the treatment group reported a range of 11 to 28 hours of dance training (see Table 1 for basic demographic information).

The sampling procedure used in this study was both convenient and purposive (Patton, 2002). An appropriate sample size was determined prior to the start of the study. A sample of 28 participants was deemed suitable by the primary researcher in consultation with a statistician and G*power software (Faul, Erdfelder, Lang, \& Buchner, 2007) by adopting a moderate effect size of 0.56 (Cohen, 1988) with a desired power of 0.80 .

\section{Dance Program Characteristics and Setting}

Based on self-reported data from the participants, their dance program was somewhat rigorous, requiring a number of hours of class, rehearsal, and/or performance that is comparable to the 20 countable hours put forth by the National Collegiate Athletic Association (NCAA) for 
college student-athletes. Of the 30 participants, 29 reported that they had to audition in order to be admitted into the program. When asked to describe the technical focus of their program, $77 \%$ of dancers $(n=23)$ described their program as ballet-based, and the other $23 \%$ of dancers $(n=7)$ described the program as either mixed-repertoire or consisting of more than one technical discipline (e.g., ballet, modern). Dance majors at this liberal arts institution could receive either a Bachelor of Arts degree in Dance or a Bachelor of Fine Arts degree in Dance, with the latter degree traditionally requiring additional studio work to prepare students for a professional career in the dance industry.

The program is housed in an on-campus building, which consists of several dance studio spaces, dressing rooms, and faculty/staff offices. There were pianos in the studios for times when live accompaniment was offered for class. The studios were well-lit, clean, and had flooring that was appropriate for dance conditioning and performance. Outside of the studios, there was a lounge where dancers could stretch, eat, socialize, use their phones or iPods, and complete written homework between classes. Based on the primary researcher's observations, this lounge area encouraged a sense of "community," such that multiple conversations could be held at once, and people would make the space their own by spreading out textbooks, lunch boxes, or their own bodies when they needed to recover from or prepare for a technique class. The lounge area also housed resources for students, such as exercise DVDs, stretching equipment, and dance program photo albums.

When the primary researcher arrived to the site each week to meet with the dancers in the treatment group, the lounge area served as the "home base" for the project. It was here that dancers could seek out the primary researcher for questions and concerns. One dancer used this space for a few brief individual consultations with the primary researcher about managing stress 
and expectations. Another dancer approached the researcher in the studio space to inquire about an approach to stress management that a family member had utilized. A third dancer asked the researcher for more information on health/wellness careers such as performance psychology, and the researcher shared resources in a brief in-person meeting and via email correspondence. It should be noted that many dancers in the treatment and control groups acknowledged the primary researcher's (and research assistant's) presence in the lounge, but very few asked for advice or consultation over the six-week period, particularly when in the presence of other dance students.

The primary researcher and research assistant observed that classes seemed to consistently begin a few minutes late, either due to students still transitioning from a previous class, or the faculty member needing to take a few minutes to switch focus from a meeting and/or to finalize content. The most commonly observed dance class was the one that the treatment group participants were taking (i.e., modern dance). This class was fairly structured, and it was clear from observing that the students were accustomed to this structure. Sometimes the students faced the front of the studio so that they could pay attention to the instructor and/or see themselves reflected in the wall-length mirror. Other times they faced different directions for individual or small group exercises, or stood in a circle to do community-focused dance work.

Despite the program having an emphasis on classical ballet technique, dancers did not look uniform in appearance and dress code. The dance students represented many body types and wore a range of dancewear, including leotards, tights, sauna pants, t-shirts, sweat pants, and leggings. Women's hair was typically pulled back in a loose or tight bun or ponytail. Both the primary researcher and research assistant noted the presence of coffee, tea, and water bottles placed around the borders of the dance studio, which dancers would visit during short breaks. 
Although a somewhat casual tone was established, there were clear classroom norms as well. For instance, dancers would never re-enter a studio space during an exercise or dance number. They would wait until the music stopped and a combination or section of dancing was complete. Additionally, dancers clapped for the pianist at the end of each class. They also thanked any person of authority, including the primary researcher and research assistant, before leaving.

\section{Instrumentation}

Basic demographics survey. The primary researcher developed a basic demographics survey specifically for this study in an effort to contextualize the sample (see Appendix B). Survey items included participants' age, gender, year in school, total number of years dancing, total number of hours dancing per week, and current injury status. The demographics survey also asked participants to rate their level of familiarity with the skills to be introduced during the intervention program (i.e., diaphragmatic breathing, progressive muscle relaxation, imagery, self-talk, and mindfulness). Information gathered via this instrument was intended to help describe the sample's characteristics, rather than to answer a particular research question.

Athletic Coping Skills Inventory-28. The Athletic Coping Skills Inventory-28 (ACSI28; Smith, Schutz, Smoll, \& Ptacek, 1995) is a multi-dimensional measure of self-reported psychological skills utilized in sport (see Appendix C). Seven sport-specific subscales make up the full measure, each subscale having four items answered on a 4-point Likert scale from 0 (almost never) to 3 (almost always). A total score, combining all 28 items, is used to assess overall coping skills, with a possible range of 0 to 84 . The primary researcher chose to use this survey due to its multi-dimensional measure and the fact that it had previously been used with a dance population. 
Researchers Estanol, Shepherd, and MacDonald (2013) have previously reported a mean total score of 49.34 and a standard deviation of 10.14 on the ACSI-28 for their sample of dancers $(N=205)$. Their sample consisted of collegiate-level ballet and modern dancers, professional dancers, and contemporary dancers. The researchers' descriptives are comparable to the present study's sample for both week one $(M=48.83, S D=9.79)$ and week six $(M=48.72, S D=10.66)$.

The reliability and validity of the ACSI-28 has been tested with multiple samples and has been used to study coping skills and behaviors in the sport context (e.g., Mummery, Schofield, \& Perry, 2004; Omar-Fauzee, Daud, Abdullah, \& Rashid, 2009; Smith et al., 1995). Researchers have indicated questionable to acceptable reliability for the concentration subscale, with a Cronbach's alpha of 0.62 , and good reliability for the peaking under pressure subscale, with a Cronbach's alpha of 0.78 . These statistics represent the lowest and highest measures of internal consistency across all subscales of the instrument (Noh, Morris, \& Andersen, 2007; Smith et al., 1995). Additionally, researchers reported that one-week test-retest coefficients from a sample of 90 college students and athletes had a median of 0.82 (Noh, Morris, \& Andersen, 2007; Smith et al., 1995).

In the present study, the primary researcher examined five-week test-retest reliability of the ACSI-28. The correlation between ACSI-28 total scores collected during the first and sixth weeks of the study was strong, $r=0.756, p=0.000$. Additionally, the primary researcher observed the relationship between pre- and post-intervention total scores on this instrument by group condition. There was a strong relationship between pre- and post-intervention ACSI-28 total scores for the treatment group, $r=0.870, p=0.000$. There was also a statistically significant correlation between these scores for the control group, $r=0.555, p=0.039$. These 
correlations are relatively consistent with the reliability statistics reported by the instrument's developers (Smith, Schutz, Smoll, \& Ptacek, 1995).

As for convergent and discriminant validity, the developers reported moderate correlations between the ACSI-28 and other similar instruments. These instruments included the Self-Control Schedule (Rosenbaum, 1980) $(r=0.44)$, Sport Anxiety Scale (Smith, Smoll, \& Schutz, 1990) $(r=-.43)$, and Self-Efficacy Scale (Coppel, 1980) $(r=0.58)$. In contrast to other coping questionnaires that are non-specific to sport (e.g., COPE; Carver, Scheier, \& Weintraub, 1989), the ACSI-28 was developed specifically for use with athletes. However, it should be noted that the instrument is not grounded in one or more theories of stress and coping (Croker, Kowalski, \& Graham, 1998), but may be considered as having originated from a cognitive behavioral approach (Estanol, Shepherd, \& MacDonald, 2013).

For the purpose of this study, some of the items were modified to reflect a dance-specific context. The words "coach" or "manager" were replaced with "instructor" or "choreographer." Similar changes have been made to the ACSI-28 by others in their research with dancers (e.g., Estanol, Shepherd, \& MacDonald, 2013; Noh, Morris, \& Andersen, 2007). Estanol and colleagues (2013) reported a Cronbach's alpha of 0.84 for the ACSI-28 when modified and used with their sample. Noh and colleagues (2007) reported slightly lower reliability with these modifications. However, the ACSI-28 had been translated into Korean, and so the translation could also have influenced their reliability estimates.

In the present study, a Cronbach's alpha of 0.727 was observed for week one across 30 participants, and a Cronbach's alpha of 0.741 was observed for week six across 29 participants. As for the subscales, internal consistency coefficients ranged from a low of 0.508 for the confidence and achievement motivation subscale, to a high of 0.861 for the peaking under 
pressure subscale (see Table 2 for internal consistency at week one compared to the developers' alphas). These numbers are fairly consistent with the findings of Estanol, Shepherd, and MacDonald (2013). They reported low internal consistency for one subscale (i.e., coachability), with all other subscales having a Cronbach's alpha greater than 0.65 . This was also the case in the present study, although the subscale below this 0.65 cut-off point was confidence and achievement motivation, instead of coachability.

Pain Appraisal Inventory. The Pain Appraisal Inventory (PAI; Unruh \& Ritchie, 1998) is a self-report instrument comprised of 16 items that quantifies and classifies individuals' beliefs about physical pain. The PAI also helps to evaluate what respondents think about their pain, and how these thoughts make them feel (see Appendix D). Based on literature in stress and coping research, the developers of the PAI included two scales within this instrument: threat appraisal and challenge appraisal. Threat was defined as "anticipated or actual physical or psychological harm, loss, injury or damage associated with a pain event," while challenge was defined as "a test of strength, endurance or abilities, with the potential for growth, mastery or gain associated with a pain event" (Unruh \& Ritchie, 1998, p. 106). One might expect these two subscales to be negatively related. However, researcher Anderson and Hanrahan (2008) reported the relationship between threat and challenge to be weak to moderate $(r=0.41)$ in a sample of dancers $(N=51)$ who represented a variety of styles and training levels.

Each subscale contains eight items that are scored on a six-point Likert scale from 1 (strongly disagree) to 6 (strongly agree). A total composite score for pain appraisal can be calculated by summing the 16 items, with a possible range of 16 to 96 . The sum of each subscale can also be calculated, with a possible range of 8 to 48 . However, it should be noted that the developers stated that they initially considered a rating of " 3 " or higher to indicate an appraisal 
of pain (Unruh \& Ritchie, 1998), and so the total scores and possible ranges for this instrument and its subscales may be highly dependent on the severity and duration of pain that participants are experiencing when this survey is administered.

Previously, Anderson and Hanrahan (2008) have reported a range of 8 to 43 for the threat subscale, and a range of 8 to 41 for the challenge subscale. No total scale psychometrics were reported in their study. Compared to Anderson and Hanrahan's (2008) sample, the present study's participants reported a similar range of scores on the PAI subscales. The threat subscale had a range of 8 to 37 for week one $(M=18.31, S D=9.35)$ and a range of 8 to 32 for week six $(M=18.68, S D=7.94)$. Similarly, the challenge subscale had a range of 8 to 42 for week one $(M$ $=20.62, S D=10.27)$ and a range of 8 to 43 for week $\operatorname{six}(M=21.03, S D=9.52)$.

The instruments' developers reported evidence of good validity and reliability. Concurrent criterion validity was determined by comparing the PAI's properties to other pain instruments, including the McGill Pain Questionnaire (MPQ; Melzachk, 1987) and the Pain Disability Index (PDI; Tait, Chibnall, \& Krause, 1990). Using data collected from a sample $(N=$ 46) of college students, health professionals, and community members, the developers reported that the threat subscale yielded a Cronbach's alpha of 0.86 and the challenge subscale yielded a Cronbach's alpha of 0.81 . In the present study, internal consistency was also strong. Cronbach's alpha for the PAI at week one was 0.881 . At week six, Cronbach's alpha was 0.886 . When observing the two subscales separately, the threat subscale had a Cronbach's alpha of 0.914, and the challenge subscale had a Cronbach's alpha of 0.904.

The developers of the PAI have discussed some of the instruments' limitations. Among these limitations is the unexplored relationship between appraisals, and chronic versus acute pain; the developers have suggested that differences in type of pain and their direct or indirect 
effects on appraisal is unknown. The instrument's developers have also reported a lack of testretest reliability. Given this fact, the primary researcher in the present study decided to run correlations on the PAI total scores as well as both subscales across all participants. The correlation coefficient for the PAI total scores was moderate, $r=0.399, p=0.035$. When observing PAI threat subscale scores, no meaningful relationship was observed between the first and second set of scores, $r=0.111, p=0.575$. In contrast, the PAI challenge subscale scores were strongly correlated, $r=0.527, p=0.004$ (see Table 3).

When examining test-retest reliability by group condition, there were slightly different findings. The relationship between pre- and post-intervention PAI total scores in the control group was weak, $r=0.229, p=0.430$. The threat subscale showed no relationship, $r=-0.196, p$ $=0.502$, and the challenge subscale showed a moderate relationship, $r=0.497, p=0.071$ (see Table 4). In contrast, the pre- and post-intervention PAI total scores for the treatment group showed a strong relationship, $r=0.702, p=0.005$. The threat subscale data showed a weak relationship, $r=0.336, p=0.240$, and the challenge subscale data showed a moderate relationship, $r=0.664, p=0.010$ (see Table 5).

Injury tracking survey. The primary researcher developed an injury tracking survey specifically for this study (see Appendix E). The content of this survey was inspired by several sources, including an injury tracking form developed by members of the Dance/USA Task Force on Dancer Health, and researchers Noh, Morris, and Andersen (2007). Previous research suggests that "dancers may under report injuries on surveys," and, therefore, multiple questions about injury were presented (Thomas \& Tarr, 2009, p. 51). Dancers were asked to report their current injury status; where their injury or injuries occurred on the body; if the injury or injuries caused them to miss or modify any training, rehearsals, or performances; whether they sought 
medical attention; and the presence and severity of pain they may have experienced. Participants in the present study were incentivized to submit their injury reports every week throughout the study in order to be eligible to receive a $\$ 100$ Visa gift card via a raffle system.

The National Collegiate Athletic Association (NCAA) has defined a physical injury by focusing on three specific criteria. First, the injury must occur within the training or competition setting, and not in a different environment. Second, the injury must require the attention of an athletic trainer or physician affiliated with the particular group/team. Third and finally, the injury must restrict or prevent the athlete's participation in sport for at least one day subsequent to the day in which the injury occurred (Dick, Agel, \& Marshall, 2007). Due to the fact that dancers may be inclined to keep their injuries hidden, the primary researcher adopted a modified version of the NCAA's third criterion. Specifically, any self-reported time lost was considered an instance of injury, rather than having dancers wait until the following day to "start the clock" on minutes or hours of dancing missed.

Dance Experiences Survey. The Dance Experiences Survey (Krasnow \& Mainwaring, 1990) was used in the present study to capture stressors that individuals may experience as a result of their role as dancers (see Appendix F). The instrument consists of 48 items, and asks participants to use "Yes" or "No" to indicate if each item applies to them at the present time. For those items that do apply, participants indicate the impact that these stressors have on them from -3 (extremely negative) to +3 (extremely positive). The instrument begins with an example so that participants are clear on the instructions and the format of the survey. At the end of the 48 items, participants are provided space to write-in any other stressors that they are experiencing, and to report the perceived impact of these stressors. The developers did not provide psychometric data via personal correspondence with the primary researcher, and there is a lack of psychometric 
data available in the literature that uses this survey. As such, the information gathered via this instrument was intended only to describe the sample.

Intervention program. The short-term psychological skills intervention program consisted of six sessions, with one session being conducted each week for the treatment group. This six-week time frame was determined through a search of the literature focused on shortterm behavior change and psychological skills interventions in sport (e.g., Bell, Skinner, \& Fisher, 2009; Johnson, 2000; Meichenbaum, 1993). The primary researcher developed the intervention program content in collaboration with other sport psychology and counseling professionals, and from the content presented in the existing research literature. Face and content validity was confirmed by meeting with a licensed counselor and counselor educator to review all materials (see Appendix $\mathrm{G}$ for an overview of the intervention program protocol).

Additionally, pilot testing was completed on all activities and handouts with two volunteer undergraduate students who reported a background in dance. During pilot testing, these two students provided verbal and written feedback about the aspects of the program's content that they liked and did not like, and what they thought could be improved upon before its delivery to treatment group participants. The primary researcher compiled this feedback in written format and used it as a guideline for finalizing all intervention program materials. The primary researcher also asked these two students to complete all self-report surveys so that an estimated time period could be established for the data collection packets to be administered during the first and sixth weeks of the study.

All sessions followed a similar outline, with exception of the first and last, which focused on data collection procedures. For the second through fifth weeks, the primary researcher's routine for each session consisted of: (1) having the participants review what they had learned or 
practiced in the previous week, (2) introducing the new skill to be learned or practiced, and then (3) providing an experiential learning activity focused on the week's topic. After each skill was introduced and practiced, participants reflected on their experiences. For instance, participants were encouraged to pose questions or concerns about the topic or activity and to share their thoughts on how to use the skills(s) in dance class or other life pursuits.

The first skills introduced were diaphragmatic breathing and progressive muscle relaxation. Participants were led through a deep breathing script and then through a progressive muscle relaxation exercise (in either an active or passive manner). The next skill introduced was imagery. Participants were led through a relaxation and coping imagery script based on Jon Kabat Zinn's Mountain Meditation, and also led through a performance imagery script intended to help them prepare for dance class. The fourth week's focus was on cognitive reframing and self-talk. Participants were given a handout that contained many negative, maladaptive, or irrational statements related to dancing. In small groups, they came up with more positive, adaptive, and truthful statements to use when they are dancing. The final skill introduced was mindfulness. Participants were led through a mindfulness-based stress management script that asked them to focus on their breath, a small item in their hand (a rock, provided by the primary researcher), and the present moment (see Appendix $\mathrm{H}$ for all of the exercises and activities used in the intervention program).

Throughout the intervention program, the dancers' class instructor remained present in the dance studio for all discussion, exercises, and activities. The instructor did not observe the sessions, but rather participated in the same way as the treatment group participants. However, the instructor did not participate in any data collection procedures during weeks one and six, and did not take part in the post-intervention focus group discussion. 
For each week of the intervention program, the primary researcher sent the course instructor the written scripts used for each session, as well as audio-recordings of the respective exercises or activities. The instructor offered to post these files in an online portal, to which the treatment group participants had access throughout the semester. Audio-files were created via QuickTime software and could be downloaded and listened to with an iTunes account.

Adherence journal. A weekly adherence journal was developed by the primary researcher and loosely based on the journal that Noh, Morris, and Andersen (2007) used in their study with Korean dancers (see Appendix I). Participants received a link to a secure, online survey so that they could complete the items on their own time following weeks two through five of the program (i.e., dance students were asked to submit an adherence report four times throughout the study). The survey asked participants to indicate which skill(s) they were practicing, and for what amount of time they were practicing them. Participants were also prompted to rate their efforts to practice the skill(s) on a 4-point Likert scale from 1 (no effort) to 4 (excellent effort). This particular item was intended to capture their perceived level of investment in the program. Findings from this instrument are reported as exploratory analyses following the results of the main research questions.

\section{Procedure}

The primary researcher contacted several dance program chairpersons in the Mid-Atlantic region of the United States by email. The email to these chairpersons contained a brief proposal of the research study and its anticipated timeline. Once a dance professor expressed interest in the study, a phone call was scheduled to talk to this professor one-on-one to further explore his/her interest. Then, the primary researcher and the interested professor assessed the feasibility of recruiting another professor from the same department who might also be interested in the 
study. Once this second person was identified, the two professors and the primary researcher developed a schedule that worked for all parties involved.

The primary researcher obtained Institutional Review Board (IRB) approval from the primary institution and completed supplemental documents for the approval of the institution at which data collection procedures occurred. These latter documents included an IRB Authorization Agreement and letters of permission from each classroom instructor. The research team — which consisted of the primary researcher, an advisor, and two research assistants — then finalized all instrumentation and the intervention program content.

For six consecutive weeks, the primary researcher traveled from her primary institution to the data collection site. During weeks one and six, data collection was the main focus. At the start of the first week, the primary researcher briefly explained the research design to all participants so that they understood how group assignments were made. Participants completed the Athletic Coping Skills Inventory (ACSI-28; Smith, Schutz, Smoll, \& Ptacek, 1995), the Pain Appraisal Inventory (PAI; Unruh \& Ritchie, 1998), and an injury tracking measure developed specifically for this study for the first and sixth weeks. In addition, two instruments were used to contextualize the sample, and were collected during week one. These included a basic demographics survey and the Dance Experiences Survey (Krasnow \& Mainwaring, 1990), the latter of which was intended to capture perceived levels of stress in dance-specific situations. A research assistant accompanied the primary researcher for weeks one and six so that the data collection procedures could be viewed by participants as a somewhat separate process from the delivery of the intervention program itself.

To ensure confidentiality throughout the study, participants who volunteered to take part in data collection procedures used code names. These code names were developed during week 
one of the study with both groups, and consisted of two letters and four numbers. The creation of code names was necessary given the repeated measures nature of some of the surveys. The participants' contact information (i.e., phone numbers and email addresses) was also voluntarily provided during the first week of the study, and these details were stored as a separate file on a password-protected computer throughout the course of the study. Participants' contact information was necessary in order to collect data for weeks two through five (see Appendix J). Emails were sent through Gmail and text messages were sent using EZ Text, a messaging service that allows recipients to opt-in or opt-out of correspondence at any time.

For weeks two through five, the control group did not meet with the primary researcher. Participants in this group were sent the injury tracking survey via email or text message. The treatment group also received the injury tracking survey via email or text message during this same time frame. In contrast to the control group, the treatment group met weekly with the primary researcher to learn and practice the psychological skills previously described.

While at the site, the primary researcher took field notes to capture the overall context of the dance program and the setting in which the participants were learning and training. The primary researcher also arrived early to each session to observe other classes and to be available to answer participants' questions. After each session with the treatment group, the primary researcher remained on-site to address questions and/or comments. In addition to receiving the injury tracking survey on a weekly basis, participants in the treatment group received an email or text message that contained a secure link to the weekly adherence journal. The purpose of the adherence journal was to capture participants' efforts to practice the skills introduced throughout the intervention program. 
At the end of the intervention program — and once data collection procedures for week six were complete - the research assistant conducted a focus group discussion with participants in the treatment group (see Appendix K for semi-structured interview guide). This discussion was audio-recorded so that all responses could be fully transcribed. Focus group participants were incentivized with the offer of a chance to receive a $\$ 50$ Visa gift card via a raffle system. Participants were asked to describe: (1) what went well, (2) what didn't go well, (3) what was surprising or new, (4) how participants could use information provided in the intervention in their lives, and (5) what about the delivery (and/or the deliverer) would they change.

Following the focus group discussion, the primary researcher conducted two exploratory interviews (i.e., stakeholder interviews) with each of the course instructors who allowed the researcher access to their classrooms. The instructors were interviewed via phone, and the interviews were audio-recorded so that they could be subsequently transcribed. Both instructors were informed that their names and titles would not be affiliated with the results and that any identifying information would be removed from the transcriptions. These interviews focused on gathering the instructors' thoughts on their institution, program and teaching philosophies, and investment in the research topics and/or process. Interviews ranged from approximately 30 to 50 minutes and followed a semi-structured interview guide (see Appendix L for a summary of findings from these interviews).

Following the conclusion of the study, the primary researcher remained available to participants and their instructors for follow-up questions or concerns. A basic summary of results was also provided. Finally, the primary researcher worked with the instructors to make the intervention program content available to those dancers who were initially assigned to the control group. 


\section{Data Analyses}

Before any analyses were conducted, the researcher cleaned the data set by looking for impossible values, missing data, and cases of attrition. A few issues were found. The weekly injury reports had the highest response rate at the start of and end of the study, and the lowest response rate for the weeks in between. Two inconsistent or not plausible cases were identified within the injury data. Three participants reported their injury status either twice in the same day or twice in the same week. Given the timing of these submissions, the primary researcher made the decision to interpret the first of the two submissions as the report for the previous week, and the second of the two submissions as the report for the current or upcoming week. No impossible values were discovered within the data, and no other major issues were discovered.

Multiple statistical analyses were used to answer the proposed research questions. Basic descriptive and frequency statistics were run to contextualize the sample used in the present study. Then, to answer the first research question, an ANCOVA was run on the post-test data from the ACSI-28, using pre-test ACSI-28 scores as the covariate. This analysis was run on the total scores for the ACSI-28 as well as for the seven subscales of the instrument. The significance level was set to $p=0.05$, with the goal of observing a small to moderate effect size.

The second research question was answered by running a second ANCOVA on the posttest data from the PAI, using pre-test PAI scores as the covariate. This analysis was run on the PAI total scores, as well as for the two subscales of the instrument. The significance level was again set to $p=0.05$. The primary researcher chose these statistical analyses based on the fact that ANCOVA may be used to increase statistical power and may potentially help with adjusting pre-test differences between the non-random groups used in the present study (Harlow, 2014). It should be noted that the use of ANCOVA to statistically control for a covariate is a somewhat 
controversial approach, and therefore statisticians recommend that results be interpreted with caution (Harlow, 2014; Miller \& Chapman, 2001)

The primary researcher planned to run repeated measures analysis of variance (ANOVA) to answer the third research question. This specific test could allow for the examination of the average frequency and duration of self-reported injuries throughout the intervention program. However, this analysis could not be run for two primary reasons. First, the number of dancers who reported their injury status (and injury details) varied by week. Most or all participants reported their injuries for weeks one and six. However, during the weeks in between, the number of reports decreased. Given that repeated measures ANOVA requires information to be observed or collected on the same participants at each time-point, the missing data greatly affected the ability to conduct this analysis. Additionally, there were very few dancers $(n=3)$ who reported any time lost due to injury. Time lost could have served as the continuous dependent variable in this analysis had more injury reports, and the details within them, been submitted each week. In the absence of this analysis, basic descriptive statistics were run in an effort to report other informative findings.

As for the fourth and final research question, the primary researcher utilized qualitative analyses. The primary researcher and two graduate students followed open coding procedures (Creswell, 2007; Patton, 2002), and utilized a typological approach to identify themes and categories from the focus group discussion. This same protocol was used to analyze the two exploratory stakeholder interviews. The coding team also engaged in data triangulation (Patton, 2002) so as to determine similar and different perspectives. 


\section{Results}

\section{Descriptive Statistics}

At the start of the research study, participants in the control group had a slightly lower average score $(M=38.47, S D=15.84)$ on the Pain Appraisal Inventory (PAI) than did participants in the treatment group $(M=39.42, S D=14.77)$. In contrast, control group participants reported slightly higher coping skills $(M=52.00, S D=8.26)$ as measured by the Athletic Coping Skills Inventory-28 (ACSI-28) when compared to treatment group participants $(M=45.67, S D=10.42)$ at the start of the study.

At the conclusion of the study, participants in the control group reported lower scores on the PAI $(M=36.14, S D=16.27)$ when compared to treatment group participants $(M=43.07, S D$ $=11.49)$. For the ACSI-28, control group participants still had higher scores $(M=50.14, S D=$ 8.11) compared to treatment group participants $(M=47.40, S D=12.73)$. Overall, the control group's mean scores on the PAI and ACSI-28 slightly decreased over time, and the treatment group's mean scores on these instruments slightly increased over time (see Table 1).

Prior to the delivery of any intervention program content, participants' familiarity with psychological skills was assessed. Of the 15 participants in the control group, the majority $(n=$ 14) reported some level of familiarity with deep or diaphragmatic breathing, and over half ( $n=$

9) reported familiarity with progressive muscle relaxation. All control group participants indicated familiarity with imagery and also with self-talk or cognitive behavioral therapy. Lastly, all but one participant indicated some level of familiarity with mindfulness $(n=14)$.

In comparison to the control group, all 15 participants in the treatment group reported some level of familiarity with deep or diaphragmatic breathing and with imagery. Most $(n=11)$ reported familiarity with progressive muscle relaxation, and also with self-talk or cognitive 
behavioral therapy $(n=14)$. All but two treatment group participants were familiar with mindfulness prior to the intervention program's delivery $(n=13)$.

\section{Quantitative Results}

Is there a difference in self-reported coping skills between dancers who participated in the intervention program and those who do not? There was no significant difference found between treatment versus control group assignment on post-test ACSI-28 scores after controlling for pre-test ACSI-28 scores, $F(1,26)=1.004, p=0.326, \eta^{2}=0.037$ (see Table 6). There were also no significant differences between groups on the post-test scores for the seven subscales of the ACSI-28 when controlling for pre-test subscale scores. None of the analyses of these subscales appeared to approach significance.

\section{Is there a difference in self-reported pain appraisals between dancers who} participated in the intervention program and those who do not? There was no significant difference found between treatment versus control group assignment on post-test PAI scores after controlling for pre-test PAI scores, $F(1,25)=1.554, p=0.224, \eta^{2}=0.059$ (see Table 7). Additionally, for the PAI challenge subscale, average scores were similar between groups and no significant effect was found when an ANCOVA was run, $F(1,25)=0.222, p=0.641, \eta^{2}=0.009$. However, for the PAI threat subscale, there was a trend for post-test scores to be higher in the treatment group than in the control group, when controlling for pre-test scores, $F(1,25)=4.016$, $p=0.056, \eta^{2}=0.138$.

Is the rate and severity of injuries affected by the intervention program? There were several missing data points to run the anticipated analyses, as many participants did not report their injury status on a weekly basis despite being prompted to do so. The number of injury reports ranged from a high of 30 participants (week one) to a low of 16 participants (week five). 
Additionally, those who did report their injuries often reported not missing any class, rehearsal, or performance time as a result of these injuries. A repeated measures ANOVA was therefore not feasible to run, with only four reports of time lost due to injury across three different participants. The primary researcher consulted with a professional statistician not affiliated with the research team, who confirmed that this analysis could not be conducted with the data collected from participants.

The primary researcher therefore examined basic descriptive statistics to characterize the injury reports submitted throughout the duration of the study (see Table 8). For the first week of the study, there were four injured dancers $(26.7 \%)$ in the control group, and nine injured dancers $(60 \%)$ in the treatment group. In the final week of the study, six dancers in the control group reported one or more injuries $(40 \%)$, as compared to nine dancers $(60 \%)$ in the treatment group.

There were 129 injury reports in total throughout the six-week intervention period. Of these 129 reports, 22 contained some form of modification to training as a result of injury or pain. These modifications typically consisted of avoiding part of a class (e.g., "no pointe barre on Wednesday") or adjusting footwear or technique (e.g., "did not over turnout"). Furthermore, 86 (67\%) out of the 129 injury reports included a physical pain rating of at least minor pain. On average, dancers who reported physical pain on the injury tracking survey assessed their pain to be between minor and moderate $(M=1.56, S D=0.57)$. However, the intensity of physical pain was reported at all three levels: minor $(n=41)$, moderate $(n=42)$, and severe $(n=3)$.

\section{Qualitative Results}

\section{What were the dancers' reactions to this intervention program, and, more} specifically, what aspects of the program were useful and not useful, and why? The coding team individually identified themes and categories that emerged from the focus group and 
subsequently discussed the major and minor themes that were most representative of the aggregated responses. The three major themes identified by the coding team were: (1) things that went well, (2) things that could have gone better (or should be improved in the future), and (3) reflections about the primary researcher. Each major theme, and its sub-themes are discussed, with supporting quotes where appropriate.

Things that went well. Four topics emerged from the question of what went well for participants in the treatment group throughout the study, namely group cohesion, the opportunity to learn new skills, program activities and content, and materials used.

Cohesiveness of group. The group expressed positive feelings about working together during the program: "I think we all really worked as a group and that helped a lot because we call got to put our input...I think that was a good group effort because we got a bit of everyone's opinions."

Opportunity to learn new skills. Some dance students expressed their appreciation for the time taken to "dive deep" into their minds and to make the connection between mind and body, or mind and performance: "It [the program] went into some of the deeper thought processes instead of just some of the basic things [on the surface] like physical things because people talk about them a lot."

Activities or content. Dance students reported that they thought several different activities and program content were helpful to them and their development of coping skills. Among the most beneficial skills/activities were mindfulness, self-talk, deep breathing, and a team building activity used in the first group meeting to build rapport and interest. Said one student: "I think the writing of the statements [for self-talk] was most applicable for me or the most relatable because in a ballet class that's what's going through everybody's mind all the time." Another 
student reflected on deep breathing and self-talk: "For me I think the deep breathing and self-talk helped the most because it was like during stressful times and exhaustion and I know this week, tech week, I was giving my legs a pep talk...I was like 'come on legs, you can do this!' and then just like [doing] deep breathing."

Materials. The dance students provided some positive feedback on the materials, citing that the surveys used were relatively easy to navigate and were generally a good length in terms of time demands. One student expressed his/her view: "It was good because they were very short... so that was really easy...And the surveys I think they were very good because you had a bunch of questions that kind of meant the same [thing] but different orders so that was like good...you know, reliable." Another student added the feedback that the surveys served as reminders to practice skills that the class had learned in the previous week(s): "I think the little check-in survey helped to remind me to do the exercises um not that I was able to do every single one each week but it would ask 'have you practiced it' and 'how long did you practice it?' and I would go 'well actually I haven't done it yet, so I should.’”

Things that could be improved. Feedback on what could have gone better or been improved was generally represented by three distinct categories, namely the activities and program content, the materials used, and the structure and other logistics of the program.

Activities or content. Dance students expressed a desire to better understand how the skills presented and practiced could connect to real-life situations. Their feedback suggested that they had difficulty seeing the applicability of the skills to, for example, dance auditions or final exams. Secondly, students indicated that, since they are used to being physical in the dance space and their bodies are accustomed to moving quite often, more active forms of stress management and/or self-awareness could have been introduced. Said one student: "You could add more active 
ways to relax...because for me I do not do well with mindfulness or just relaxing because I will just either fall asleep or my mind will go everywhere and just...even like yoga or different ways that you're still kind of moving but relaxing into that movement." Lastly, one student stated that he/she would have like to do some shorter activities and/or learn quicker strategies for times when a dancer may not have time to complete a full body scan or do a self-talk worksheet.

Materials. Although the dance students indicated that the materials were relatively easy to navigate and respond to, they indicated that a few items caused confusion and should be clarified. Most students who provided this feedback reported that the questions regarding a new or recurring/chronic injury were confusing. They indicated that some injuries could not be distinctly classified as new or recurring, and pain often varies from day to day or week to week. Additionally, select students reported that they experienced some technological issues during the data collection process: "I haven't gotten them [the emails or text reminders from the study] for like the past two or three weeks...I forgot about them so I never brought it up." The research assistant asked this particular participant to speak with the research team after the discussion to sort out this issue.

Structure and logistics. Several dance students commented on the logistics of the program, namely (1) program's length and frequency of meetings, (2) the time of day that the program was offered, (3) the time in the semester when the program started, and (4) the setting in which the program occurred. One student stated: "I...wish that it had been more often and longer." Support for this feedback included the suggestion that the program last for "maybe a 10week period...or even an entire semester, you know once or twice a week a semester, that way we have a chance to introduce the topic and then rehearse it again as a class...I feel like I'd be more apt to do it on my own the more often I do it in class like everybody else." Another student 
indicated that he/she did not like that the program took away from regularly scheduled class time with their dance instructor, citing that "we have very limited time and we only take this class three days a week so that was kind of frustrating for me." To go along with the feedback about limited time, one student mentioned that starting the program earlier in the semester could be beneficial; he/she noted that the later in the semester, students are likely to already be experiencing higher levels of stress. Lastly, one student suggested a change in location for the program, "because...the studio is a place where we dance and we're active, and even though we do some yoga in the studio I think maybe having a different setting [for the program] would be beneficial."

The primary researcher. The conversation about the primary researcher mainly focused on personal and professional qualities. This feedback was collected when the primary researcher was not present (i.e., was intentionally removed from the focus group discussion).

Qualities or characteristics. One dance student stated: "I feel like she genuinely wanted to be here, I didn't feel like she was putting on a façade to try and get us to feel a certain way. I felt like she was a real person genuinely wanting to come in and help us." Another student shared: "I felt very comfortable sharing personal experiences with her and with the group as a whole. I mean we know each other really well but even talking about relaxation isn't something we do, I mean she just set an atmosphere that felt comfortable." Another student added: "She genuinely cared about our progress and our well-being, she really gave off that kind of vibe."

\section{Exploratory Findings}

In addition to addressing the main research questions, the primary researcher also conducted exploratory quantitative analyses on some of the data collected from participants. In particular, the primary researcher sought to examine the relationship between injury status and 
the presence of physical pain while dancing. These correlations were of interest given the fact that the subtleties within dance culture may promote pain tolerance (Anderson \& Hanrahan, 2008). When observing week one data, there was a strong, positive relationship between injury status and pain across all participants, $r=0.669, p=0.000$. This relationship was slightly higher for the treatment group, $r=0.770, p=0.006$, than the control group, $r=0.617, p=0.025$. At week six, a similar relationship was observed between injury status and presence of physical pain while dancing across all participants, $r=0.570, p=0.002$. Interestingly, however, a significant correlation was observed for the control group, $r=0.707, p=0.010$, but not for the treatment group, $r=0.372, p=0.190$.

The primary researcher also examined which injuries prevented dancers from continuing with their normal dance training. The descriptive data showed that the injuries which required dancers to take time off were ankle sprain (10+ hours), pulled biceps tendon ( 4 hours), and chronic tendonitis ( 0.5 hours). Overall, dancers sustained a variety of injuries that affected several areas or regions of the body (see Table 9). In total, there were 109 instances in which dancers reported that their bodies had been affected by injury. Most injuries affected the lowerhalf of the body (82.6\%), with the lower leg and/or Achilles tendon (22 instances) being the most common area reported.

Dance experiences. As previously discussed, the Dance Experiences Survey lacks psychometric data and therefore was used only to characterize the sample used in the present study (see Table 10 for an overview of the top ten stressors reported across participants). For instance, participants' responses to the survey indicated that managing time was the most prevalent stressor (i.e., all but one participant said that time management was a current factor in 
their lives). Of the 29 participants who said that they were impacted by time management, 13 reported a negative impact, six reported a neutral impact, and 10 reported a positive impact.

The second most prevalent stressor (i.e., all but two participants said it was a current factor in their lives) was physical characteristics. This could range from their body type, height, or facial features, and is not specified by the survey's developers. Of the 28 participants who indicated physical characteristics to be factor to be influencing their lives, six reported a positive impact, two reported a neutral impact, and 20 reported a negative impact.

The primary researcher also examined those stressors that had the most frequent ratings of extremely positive or extremely negative. Financial status and physical characteristics were the two stressors that were rated as "extremely negative" by nine participants. Not being selected as a dancer (presumably for a performance) received an "extremely negative" rating 10 times. In contrast, 13 participants rated performing as "extremely positive," and 12 participants rated relationships with teachers and performing above expectations as "extremely positive."

Adherence to the program. The adherence journal entries that treatment group participants were asked to complete on a weekly basis provided additional information about the dancers' subjective investment in the program. Participants in the treatment group submitted a total of 44 reports, which represents $73 \%$ of total reports possible (i.e., 60 reports across the four weeks in which skills were introduced and/or practiced). The primary researcher ran basic descriptive and frequency statistics on the data collected via these journal entries and found that deep breathing was the most practiced skill, and the least practiced skills were mindfulness and progressive muscle relaxation (see Table 11 for an overview of self-reported skills practiced). Most dancers $(88.6 \%)$ reported practicing skills for a range of one and 30 minutes, with a few dancers $(n=2)$ reporting no practice and a few $(n=5)$ reporting more than 31 minutes of 
practice in a given week. With respect to any materials that dancers used to practice on a weekly basis, the most commonly reported materials were none, music, and a friend or classmate.

Overall, participants reported their effort in practicing these skills to be either "some effort" or "good effort" (41 weeks out of a total 44 weeks reported).

The primary researcher also compiled the write-in comments that students offered about their psychological skills practice outside of the group meetings. In particular, one or more students indicated that they (a) used walking and lying down to practice breathing and progressive muscle relaxation, (b) paired self-talk with reading the Bible, and (c) found breathing to be the easiest and most on-the-go skill. Reasons for not practicing skills included (a) taking time to recognize the need for relaxation, (b) forgetting to practice, and (c) experiencing undesirable or uncomfortable feelings or sensations and thus consciously avoiding practice.

\section{Discussion}

Findings from the present study suggest that the six-week intervention program had little to no significant effect on participants assigned to the treatment group, at least from a quantitative perspective. In particular, there were no significant findings related to the Athletic Coping Skills Inventory (ACSI-28) and its seven subscales. The lack of statistically significant findings with this instrument allowed the researcher to conclude that the short-term psychological skills training program did not have the intended impact of increasing dancers' coping skills, although it should be noted that the mean scores for the treatment group did increase over time.

The lack of statistically significant findings stands in contrast to the work of Noh, Morris, and Andersen (2007), who reported an increase in coping skills among the dancers in their study who participated in a 12 -week intervention program and then a subsequent 12 -week practice 
period. Nevertheless, it should be noted that Noh and colleagues (2007) utilized a gain-score approach when analyzing the data, which is a different approach than the one used in the present study. Researchers have written about the choice between using analysis of covariance (ANCOVA) versus gain-score analysis (Knapp \& Schafer, 2009), and indicated that the research question asked and the researchers' analysis preferences may be strong deciding factors.

Moreover, Noh and colleagues (2007) engaged in "pre-screening" of participants. They identified the 30 dancers who had the lowest ACSI-28 scores and then randomly assigned them to either a broad-based coping skills condition or an autogenic training condition. In the present study, the primary researcher did not group dancers with the lowest ACSI-28 scores together with the intention of subsequently seeing a significant effect. Group were already identified based on the course in which participants were enrolled for the current semester. Lastly, it is difficult to make direct comparisons between the studies given that Noh and colleagues (2007) utilized a control group that consisted of dancers from a different dance studio. In the present study, the control group consisted of dancers enrolled in the same dance program.

One potential explanation or interpretation of the findings can be found in literature focused on experimenter and/or participant expectancies. Researchers have previously stated that "the content of the questions asked may themselves stimulate new thinking" (McCambridge, Witton, \& Elbourne, 2014, p. 275). In this way, it is possible to consider that the present study's results were a product of behavior change in progress. Previous research would generally indicate that enhanced coping skills may be expected in one or more treatment groups over time (e.g., Noh, Morris, \& Andersen, 2007). However, it is possible that the introduction of new or more complex psychological skills may have altered the dancers' judgments about their 
capacities to manage stress and other challenges, or the effectiveness of their current coping strategies.

It is also worth noting that familiarity with skills prior to the intervention program delivery could have affected the study's results. As previously indicated, $73 \%$ or more of the treatment group participants reported some degree of familiarity with the skills introduced and practiced during the intervention program. Had the dancers been less familiar with these skills, it is possible that the results would demonstrate a more dramatic shift in self-reported coping skills. This explanation relates back to Noh, Morris, and Andersen's (2007) intervention-based research, as their research team strategically selected the dancers $(n=30)$ who reported the lowest levels of coping skills to be assigned to their two distinct treatment groups.

In her unpublished master's thesis, Klockare (2013) found that all of the dancers in a sample of Swedish dance academy students $(N=51)$ used a variety of psychological skills training (PST) to some extent (i.e., self-talk, emotional control, goal setting, imagery, activation, and attention control) prior to receiving any PST intervention. The one exception, she reported, was relaxation strategies, which the dancers reportedly used "rarely" (p. 2). Klockare (2013) found very few statistically significant differences in dancers' PST usage when comparing preand post-intervention data. Klockare's intervention was comprised of four distinct psychoeducational sessions, and much like the present study, was delivered by the primary researcher to group of dance students. Also similar to the present study's findings, Klockare (2013) reported that the Swedish dancers had trouble applying the psychological skills to individual pursuits outside of the classroom context. Thus, it is evident that familiarity with skills prior to receiving a PST intervention, and perhaps preferences for or against certain skills, may impact observed effects among dancers. 
The present study's results may also have been influenced by logistics, namely timing, frequency of meetings, and length. These factors, separate and taken together, may have made it difficult to capture the intended outcomes. It is conceivable that dancers in both the treatment group and the control group were already experiencing some stress given the time in the semester (e.g., upcoming exams, performances). Additionally, for those who were approaching graduation, going on auditions or seeking out employment opportunities may have put demands on their time and energy. Finances and physical characteristics were two common, negative stressors presently affecting this sample of dancers. Both of these stressors have ties to dancers' career development and aspirations.

It is possible that the intervention program content may have had a different effect on dancers' coping skills if the intervention program had different logistics. In particular, the intervention could have started earlier in the term, consisted of more frequent meetings, and been delivered over a longer period of time. Dancers in the present study used the focus group discussion to communicate their thoughts on the length of the intervention program. Participants' recommendation to have the program last for a longer period of time — and include more frequent meetings - are somewhat consistent with Noh, Morris, and Andersen's (2007) collaboration with Korean ballet dancers.

Noh and colleagues' (2007) intervention program was four times the length of the present study's timeline, and involved increased teacher/staff involvement. It is possible that, with more time and more assessments, the observed small increases in ACSI-28 scores for treatment group participants may have resulted in significant findings. Nevertheless, it should be noted that other intervention programs (e.g., Johnson, 2000; Johnson, Ekegren, \& Andersen, 2005; Maddison \& Prapavessis, 2005) that were of shorter length produced statistically significant results, 
suggesting that timeline may be one factor-but not the only factor- to consider. In the future, it is recommended that researchers and applied practitioners evaluate participants' initial interest prior to delivering an intervention, potentially in the form of a needs-assessment or a preintervention focus group discussion. This could capture participants' desired logistics (time frame, frequency of meetings, location, etc.) as well as their familiarity with psychological skills.

In addition to the non-significant findings related to coping skills in the present study, were no statistically significant findings stemming from the analyses run on the Pain Appraisal Inventory (PAI). There are many potential factors that could have led to this observation. Perhaps the most widespread and influential factor may have been the culture that exists within the dance program, and the dance population on the whole. Researchers have suggested that the desire for peer or mentor approval, the drive towards perfection, and the likelihood of a rather short career promotes avoidance of medical attention and a prolonged tolerance of pain or injury (Krasnow, 2005). Cultural and subcultural factors, such as pain tolerance, may be strong determinants of self-reported pain appraisals. If so, then it is not surprising that dancers in the treatment group did not report major changes in their appraisals over a six-week time period.

In their special issue on the complexities of pain in dance, Molnar and Karin (2017) explore the history of pain tolerance among dancers and their teachers. In particular, they suggested that "the cultural expectation that dancers would, and even should, experience pain led generations of teachers to encourage young students to endure pain as proof of their dedication to the art form" (p. 3). They also stated that dancers nowadays are becoming aware of this faulty logic, and the problematic "no pain, no gain" explanation across all situations, but the expectation nevertheless has deep roots in dance contemporary culture. 
The observed trend towards significance for the threat subscale of the PAI among treatment group participants could indicate that dancers were failing to accept their existing injuries. Or, perhaps they were appraising both new and recurrent injuries as threats to their next plans. Given that dancers in the treatment group were of junior- and senior-year status, it is possible that they viewed the acknowledgment of pain as counterproductive to their post-college goals. These goals might include auditioning, beginning a new job, or teaching dance. As previously described, dancers may feel pressure to gain stable employment in an effort to avoid financial strain (Kelman, 2000; Wainwright, Williams, \& Turner, 2005) and potentially to affirm their maturing sense of self (Brewer, Van Raalte, \& Linder, 1993; Hall \& Hill, 2012).

Furthermore, additional clarity as to the distinction between the subscales (i.e., threat and challenge), could offer insight into participants' responses. Unruh and Ritchie (1998) have stated that "threat appraisals may carry an increased likelihood of anxiety and depression, whereas challenge appraisals may be central to adequate management of chronic health problems, such as pain” (p. 109). They have also explained that as acute pain, or any form of 'new' pain, persists, appraisals are likely to become more stable or consistent (Unruh \& Ritchie, 1998). This explanation has implications for the interpretation of the present study's findings. Specifically, the observed trend for post-test scores to be higher in the treatment group than in the control group might suggest that treatment group participants' responses were influenced to some (unknown) extent by social factors. These factors could include the motivational climate of their dance program, the inherent pressures in the dance community, or perhaps perceived expectations of the researcher throughout the study.

Anderson and Hanrahan (2008) have stated that dancers often have a history of performing in pain, and these experiences reinforce its normalcy. In their study of pain and pain 
appraisals with a sample of dancers $(N=51)$, the researchers wrote that "dancers will appraise all pain experienced in a similar manner, regardless of the severity or if the pain is a warning sign of serious injury" (p. 14). They explained that cultural pressures or expectations might promote dancers' continued training and performing in a relatively uniform manner across all painful experiences, since they have internalized this norm and potentially found success, praise, or both, in doing so.

Markula (2012) also addressed this uniformity in cognitive appraisals of pain, stating that semi-professional contemporary dancers may demonstrate difficulty determining what is pain and what is injury. This might be especially true if dancers have internalized the belief that pain is an indicator of the body working or improving. Dance students in the present study may also have appraised their pain and/or injuries as normal or 'to be expected' given the regions of the body where most injuries were reported. Over two-thirds of their weekly reports identified the lower-half of the body as being affected by pain or injury. This finding is consistent with the dance medicine, and more specifically, dance injury, literature (Russell, 2013). Dancers may, in a way, have justified their lower-body pain by reminding themselves that standing, turning, and jumping on the tips of one's toes is unnatural, and bound to cause some degree of discomfort.

Anderson and Hanrahan (2008) have further claimed that dancers may need to develop the capacity to differentiate between types of pain. In other words, dancers do not necessarily know how to discern pain that is to be expected from training or rehearsal, and pain that is related to injury and needs to be treated by a health professional (Anderson \& Hanrahan, 2008). Until they become aware of the differences, dancers may, by default, make the choice to push through. This may be especially true for female dancers, who are often competing against one 
another for the same roles, regardless of the training level or intensity of the university program or professional company.

It is worth noting that, in the present study, the strong, positive correlation between preand post-intervention PAI total scores for the treatment group were more consistent, in comparison to the control group. This finding could indicate that treatment group participants were more honest than control group participants in their reports of perceived pain, even if social factors, such as those previously discussed, were at play. This interpretation should nevertheless be considered with caution, given that there is limited information available on the test-retest reliability for the PAI. Based on the psychometrics found in the present study, the appropriateness of its use should be further investigated, particularly with a dance population.

It also may be possible that the intervention program's length was insufficient in terms of giving the dance students enough time to move from a threat appraisal to a challenge-focused appraisal. For instance, the strong correlation between challenge subscale scores for participants in both groups could be indicative of appraisal stability. Nevertheless, when examining correlations within the respective groups, it is possible that individuals with chronic pain may have shifted from a threat to a challenge mindset in an effort to continue dancing in their classes, rehearsals, and performances. Essentially, they were "pushing through" their pain, as has been discussed by many researchers to date (e.g., Andersen \& Hanrahan, 2008; Krasnow, 2005; Noh \& Morris, 2004).

In the present study, the primary researcher sought not only to capture pain appraisals, but also to examine dancers' self-reported injury frequency and severity across the six-week time frame. Ultimately, the third research question could not be directly answered for methodological, and potentially social or cultural, reasons. The materials that were developed and utilized to 
assess injury were a hindrance for some students, especially when reporting during weeks two through five. Based on their feedback in the focus group discussion, dancers in the treatment group were confused about how to answer some of the questions in the weekly injury report. They also indicated that at times they were unsure as to whether an injury should be classified as new or recurrent. This may be a further reflection of the dance culture, which often is comprised of injuries that return due to overuse (Ekegren, Quested, \& Brodrick, 2013), and could be classified as new or recurring depending upon a dancer's full medical history.

Overall, selecting an appropriate way to measure injury was a challenge. A time-loss definition was insufficient to run analyses, with only three out of 30 participants taking any time off due to injury for the duration of the study. This definition states that an injury has occurred when a participant is forced to modify or stop their training for a set period of time, such as a class, rehearsal, or performance (Malisoux, Frisch, Urhausen, Seil, \& Theisen, 2013). The 129 injury reports submitted by participants often provided minimal information about one's injury experience. For example, dancers could choose to reply "Yes" or "No" to indicate if they were injured for a particular week. As such, this item was a forced-choice, binary variable that could not be used to run repeated measures ANOVA. Researchers have suggested that many dancers will continue to train or perform in spite of an injury (Anderson \& Hanrahan, 2008). Therefore, capturing physical pain and/or injury status on a continuous scale, and not focusing solely on time-lost, is a recommendation for future research with this population.

Unfortunately, alternate definitions to the one previously described still use time as a major factor in determining a student's injury status. The National Collegiate Athletic Association (NCAA)'s definition of injury places emphasis on the time lost due to injury and its associated pain. In addition, it calls for the student-athlete to be treated by a medical professional 
(Dick, Agel, \& Marshall, 2007). However, in university dance programs like the one in the present study, it is unlikely that an athletic trainer or physician is working solely with studentdancers. Therefore, dancers may be more inclined to engage in self-medication strategies or ignore the injury until it jeopardizes their health or safety, and going to a specialist or the university medical center is deemed feasible and necessary.

It is also possible that a busy schedule may have gotten in the way of dancers consistently and accurately reporting their injury status, time-lost due to injury, and current level of physical pain. For instance, there were a few cases in which dancers completed more than one injury report within a 24-hour period, suggesting that either they forgot to complete the survey, or simply did not have the time or interest. Russell (2013) has explained that college dance students may be particularly vulnerable to lack of rest due to the "combination of their dance practice, the requirements of their non-dance academic work, and, often, their need to maintain employment to cover their personal expenses" (p. 204). These demands, separately and taken together, may provide a rationale for noncompliance, particularly for the weekly injury reports as well as the adherence journal submitted by treatment group participants.

Time management has been presented as a recommended topic for NCAA CHAMPS/Life Skills programs (Ahlgren-Bedics \& Monda, 2009), and it is possible that college dance students could also benefit from personal skill development in this area. Participants in the present study identified "managing time" as one of the top ten stressors that were currently influencing their dance experiences. Another possible reason for noncompliance with the weekly injury reports and the adherence journal is the potential frustration about the intervention program being offered in place of regular dance class. This frustration was briefly mentioned by participants during the focus group discussion. 
Furthermore, self-report procedures were used to collect injury information in the present study. This approach was taken given that the dance program did not appear to have, or offer to the primary researcher, a full-time athletic trainer or physical therapist who had daily interaction with the dancers. In personal communication with the primary researcher, one of the instructors indicated that, on occasion, students are screened for potential physical and/or anatomical issues that could affect their technique and injury susceptibility. Therefore, the two options for collecting injury data were to have the dancers report injuries themselves, or require this of the classroom instructors. The dancers in the treatment group were already being asked to complete a self-report instruments on a weekly basis (i.e., adherence journal), and thus it was assumed that they would be the best source of information. This approach also put less work on the classroom instructors who had already sacrificed class time to enable the research team to collect data and the primary researcher to deliver the intervention program.

This method of voluntary reporting stands in contrast to previous research with dancers (Noh, Morris, \& Andersen, 2007), in which members of a training staff were responsible for recording injury data and subsequently providing it to the research team. This approach removes accountability, and subjectivity, from the dancer. It is possible that someone serving in a paid position may be more objective, or definitive, about whether a dancer could complete a class, rehearsal, or performance without taking time off or modifying technique or choreography. Researchers who focus on injury risk and/or rates among dancers should carefully consider the ways in which injury data can be collected, and address potential issues regarding self-reporting. Additionally, researchers may need to consider gender when looking to collect data based on dancers' self- or other-reported injury experiences. In the present study, a majority of the participants in both the treatment and control groups were female. Differences in injuries based 
on gender have been previously explored (e.g., Wanke, Arendt, Mill, \& Groneberg, 2013). For example, in their prospective, single-cohort study of professional ballet dancers $(N=52)$ over a one-year period, a research team found that the female dancers reported more first-episode injuries than did male dancers (Allen, Nevill, Brooks, Koutedakis, \& Wyon, 2012). Furthermore, females had a lower mean injury severity in terms of time-loss (4 days) than did the male dancers (9 days). Thus, it is possible that gender may have played a role in the current study's findings with respect to injury reports. However, Allen and colleagues (2011) also suggest that a dancer's age, activity level, and rank (if in a professional company) should be taken into account.

Previous research focused on gender differences in pain perception and/or pain tolerance may also be noteworthy when interpreting the results of the present study. The American Psychological Association (2005) reported that an analysis of 46 meta-analyses focused on gender similarities and differences found that men and women share more likeness than disparity for constructs such as cognitive ability, leadership, and personality. However, many researchers have generally agreed that women have a lower pain threshold compared to men (Bartley \& Fillingim, 2013; Carpenter \& Patil). However, it remains unclear whether this observed gender difference is due to biological, psychological, or social factors, or perhaps a combination of these factors.

Based on the current sample, it is plausible that a lack of difference in pain appraisal was found between the treatment and control group given that these groups were both largely comprised of young women. The fact that women tend to report experiencing pain more frequently and for longer duration is also worth noting (Bartley \& Fillingim, 2013; Carpenter \& Patil), particularly within the context of dance. There is a history of dancers 'suffering in silence' 
through pain or injury, and this norm may lend itself to a suppression of injury status reports for fear of being seen as weak or uncommitted, even for the female gender.

Still another possible injury-related factor besides gender to consider in the present study is pre-intervention injury rates. The fact that almost half of the sample was injured at the start of the study may be important to note. Had there been fewer acute and chronic injuries already being addressed, and more acute or first-episode injuries sustained during the intervention period, dancers may have felt compelled to disclose them in the weekly injury reports. For every week that dancers' injury status was collected, at least two individuals from each group (i.e., control and treatment) reported that they were currently injured. Injuries were highest during weeks one and six, with week six being close to the end of the semester. The relationship between reported injury status and perceived physical pain during the final week of the study was weak for treatment group participants. This finding may suggest that, in the dancers' minds, pain and injury do not necessarily coexist. This weak relationship may also further reflect the culture of dance, in which physical pain may be accepted as a common, normal occurrence.

Overall, the quantitative findings from this study demonstrate the complexities of capturing and assessing the impact of a psychological skills intervention program across a sixweek time period. Nevertheless, overall mean scores for both the Athletic Coping Skills Inventory-28 and the Pain Appraisal Inventory did move in the intended directions, based on the research questions and aims of the present study. While these improvements were not statistically significant, they provide some evidence that psychological skills can be taught within the dance context, and that with repetition and/or adjustments to the program content, it is possible to help dancers translate concepts from the sport psychology literature into their performance enhancement and personal development efforts. 
With respect to the descriptive findings from the Dance Experiences Survey, it is also evident that dancers could benefit from psychological skills training and life skills interventions that focus on health and wellness. The most commonly reported stressors included time management and physical characteristics. Physical characteristics were among the top sources of stress reported in Noh and Morris' (2004) qualitative study of dance-related stressors. Dancers in this study reported physical appearance — and more specifically the pressures to reduce weight, maintain a low body weight, and/or maintain a particular body type — to be a stressful personal goal. Additionally, Estanol, Shepherd, and MacDonald (2013) reported that $56.3 \%$ of dancers in their sample indicated dissatisfaction with their current weight. While dance students in the present study were taking non-ballet classes as part of the program's curriculum (e.g., modern, dance conditioning), the majority of participants indicated that their program was ballet-based. Therefore, awareness of physical appearance may be present regardless of technical emphasis. In addition to the quantitative findings in the present study, the focus group discussion that was conducted following all quantitative data collection procedures led to several insightful reactions from treatment group participants. These reactions can be used to inform sport psychology consultants and dance educators as to what dance students are looking for in a psychological skills training program. Main findings from the focus group included that dancers wanted the intervention program to be longer than six weeks, consist of more frequent meetings, and potentially begin at a different time period in the year or semester. Participants in the present study also indicated an interest in alternative stress management skills that incorporated more active ways of relaxing. With respect to overall methodology and procedure, participants expressed the desire for more reminders to practice what they had learned, despite reporting that they practiced at least some of the skills for some amount of time. 
The focus group discussion also clarified things participants liked about the program, for instance, the primary researcher's knowledge and demeanor. As discussed in the present study's results, participants expressed their liking for the primary researcher to the research assistant during the focus group discussion. Participants mentioned that the primary researcher seemed to be heavily invested in the project and also in them as people. They reported that the researcher appeared calm when delivering intervention content and was available to individual dancers if/when they had questions.

This finding is consistent with student-athletes' preferences for sport psychology consultants, as Lubker, Visek, Watson, and Singpurwalla (2012) have reported that the most preferred set of characteristics, or profile, of a consultant included "female, whose ethnicity matched that of the athlete, had high interpersonal skills, wore professional attire, had an athletic body build, had previous experience as an athlete, had a high level of knowledge about sport culture, and had earned a professional degree" (p. 471). Based on the demographic information provided by participants at the start of the study, it is possible to conclude that the primary researcher's profile met most of these items. The primary researcher does identify as female and Caucasian, and most of the sample in the present study was also female and presumably Caucasian based on observations. With respect to interpersonal skills, the primary researcher had approximately five years of teaching experience as well as a graduate degree in a counselingrelated field. Participants were made aware of the primary researcher's credentials during the first session, along with the logistics and goals of the present study.

For the remaining characteristics listed by Lubker and colleagues (2012), the primary research met most of these criteria. Perhaps the criterion that was most different from the current literature was dress code. Both the primary researcher and the research assistant opted to dress in 
a manner that was consistent with attending a dance class, as this was the attire worn by the dance students as well as their instructors. It was hoped that this dress code would signal approachability and potentially foster a sense of familiarity with the dance community. As Hamilton and Robson (2006) have discussed, a psychologist or performance consultant's credibility when working with dancers "will ultimately depend on the ability and willingness to understand and appreciate each art form's language, structure, and values in this insular community" (p. 254).

The participants in the present study were aware of the primary researcher's background in dance training, which likely contributed to their assessment of the researcher's knowledge as expressed in the focus group discussion. Dance students were also informed of the primary researcher's credentials, which included two master's degrees. Additionally, how the primary researcher spent time at the site (e.g., delivering content, socializing with dancers and faculty, observing class, preparing materials, taking field notes) may have had a positive influence on the dance students' impressions of the researcher's demeanor and character.

Another aspect of the present study that treatment group participants reported liking was the opportunity to learn new skills in a setting with their peers/classmates. The primary researcher observed the participants' initial excitement about being a part of a research study that could have implications for other dancers, as well as potentially benefitting them by building and honing a "toolbox" of mental skills. Additionally, given that the dance world is sometimes described as fairly "insular," dancers most likely felt comfortable learning and practicing skills in a setting familiar to them, and in which they have had to be physically and emotionally vulnerable before. Hamilton and Robson (2006) have reinforced this feedback, explaining that performing artists "tend to respond positively [to practicing new skills] once they understand 
that...psychological preparation may be as important as physical preparation and technique," a realization that may come at the pre-professional or college level of training (p. 257).

Interestingly, as vocal as the treatment group participants were in the focus group discussion, there was an observed disconnect between the amount of feedback that they provided in-person and the amount of attention and/or effort that they reported giving to the practice of psychological skills outside of the scheduled sessions with the primary researcher. It is important to note that a majority of dancers submitted a weekly adherence journal, however their selfreported adherence was moderate at best. Weinberg and Gould (2015) have recommended that new psychological skills be practiced, on average, for 10 to 15 minutes per day, 3 to 5 days per week. Based on dancers' reports, 30 minutes per week represented the maximum time spent practicing skills outside of the weekly meetings. Thus, they may not have been seeing the direct or indirect benefits of engaging in such practice.

Scholars, practitioners, and dance educators might consider viewing adherence and/or overall buy-in as a spectrum, with most dancers in the middle of that spectrum, and a few dancers situated on either end (i.e., not at all interested or very interested). Based on the focus group feedback and informal observations, it is reasonable to conclude that for this group of dancers, learning occurs in a community context. It is difficult, perhaps particularly towards the end of a semester or during a time of increased stress, to focus one's efforts on learning or refining mental skills - even if those skills are presented as potentially beneficial to one's dance performance and/or personal development.

A few dance students made it clear that they were not very interested in the intervention program content. One student indicated on an adherence journal submission that he/she experienced strong discomfort in one session, and therefore decided not to engage in any future 
sessions. A few other students seemed to "go through the motions" of the program but did not speak up to offer their thoughts. This could be due to personality qualities (e.g., slow to warm, shy) or circumstantial factors (e.g., stressed out, currently in therapy to learn similar skills).

These students may have been more likely to not contact the primary researcher with questions about how to complete weekly surveys, or to report that they failed to receive a survey link.

On the other hand, a few dance students showed strong commitment to the research process and to the opportunity to improve their psychological skills. For example, one student took a copy of the self-talk activity with her following that week's session, and subsequently identified Bible verses that corresponded with each statement. The student shared his/her document with the primary researcher and gave the research team written permission to share it anonymously, in case it might be helpful to others (see Appendix M). This self-initiated effort stands in contrast to the average participant, for whom individual psychological skills practice was presumably not seen as practical. Students may have found it hard to find the time; been hesitant to try new things; or preferred to have the program content build into the curriculum. Students may also have been wary of the possibility of "stuff coming up" (e.g., insecurities, trauma, anxieties) that they did not feel ready to address. Researchers have suggested that any complications that might arise from meditation practice are likely to be short-lived and mild, and capable of being addressed by a counseling professional (Leppma, 2012).

\section{Limitations}

The present study has several limitations, and these should be considered when interpreting the results. One explanation for the study's findings may be the Hawthorne effect. This effect occurs when research participants — in some way, shape, or form — alter their behavior as a result of being aware that they, or things about them, are being studied 
(McCambridge, Witton, \& Elbourne, 2014). Indeed, both the control group and treatment group participants had met the primary researcher during week one of the study. As a consequence of these in-person meetings, it is possible that participants may have reported their behavior, or actually changed their behavior, so as to be seen in a positive light.

It may also be meaningful to recognize that the dance instructors who led these dance students expressed some level of interest in, and support for, the project. In fact, the instructor affiliated with the treatment group participated in the activities and exercises with the dance students during the intervention program. Therefore, instructors' interest could have had unintended impact on the students' level of truthfulness on the instruments. The instructors' interest may have been particularly influential when it came time for dancers to report their coping skills and their injuries and/or physical pain.

It is also possible that, because the primary researcher served as the main contact person for the data collection procedures and also for delivering the intervention program content, selfreports were somehow biased. If another researcher, or research team, had been responsible for one task or the other, perhaps dancers would have been more forthcoming. Based on the primary researcher's observations and field notes, there were a few dancers who demonstrated a degree of reluctance to complete the activities and exercises. Their reasons likely varied, from not wanting to sacrifice class time to experiencing discomfort with being still, silent, or intently selfaware. Other dancers may have recognized these hesitations and interpreted them as the social norm of the group without articulating its impact on the program. Collecting information via self-report methods is, by itself, a major limitation of the present study. Without an objective third-party capturing information, dance students had the ability to choose what, if anything, they shared via the instruments and focus group discussion, and to what extent. 
Furthermore, the primary researcher utilized a relatively small sample size, particularly for observing the proposed effect size. The size of the sample was somewhat pre-determined, given the method by which the primary researcher gauged interest from college dance programs, and the average size of a college dance class. Along with this limitation comes the fact that only one sample of college-aged dancers was studied, and the sampling method was one of convenience and purposiveness (Patton, 2002). Consequently, results from this group of dancers cannot be generalized to or be representative of all dancers studying at the university level.

Additional limitations stem from the procedure followed throughout the course of the study. The study's length, intervention program content, and data collection methods for both groups represent a unique combination of factors that were inspired by previous research. As the present study is not a replication study, its results cannot be directly compared to other literature and should be critiqued in order to move the body of research on this topic and population forward. As previously mentioned, the study's length of six weeks limited the amount of data that could be collected, and ultimately this time frame may have prevented the primary researcher's ability to capture dancers' experiences of being involved in the study.

Additionally, the primary researcher could not capture the long-term effects that could have potentially occurred following the intervention program's conclusion. In contrast to the present study, other researchers (i.e., Noh, Morris, \& Andersen, 2007) have structured an intervention program to include two phases, namely an active learning phase where participants are learning psychological skills, and a follow-up phase where injuries are still recorded but no new intervention content is introduced as dancers practice the acquired psychological skills.

The intervention program content was developed by the primary researcher, who used the existing literature to identify appropriate psychological skills to use with a dance population. 
This was an inherent limitation, since the amount of evidence-based literature about developing and executing dance-specific interventions continues to be limited (Noh \& Morris, 2004). Additionally, the primary researcher did not utilize any pre-screening instruments and/or a needs-assessment with this sample of dance students before delivering the intervention program content. Had a pre-intervention survey been administered, it is possible that different instrumentation and/or methodology would have been employed. Furthermore, the primary researcher may have been better able to tailor the program content to this specific college dance program, thus potentially gaining more interest and engagement from its students.

Data collection methods were also reportedly difficult at times. During the focus group discussion, dancers expressed their confusion over some of the items that were contained in surveys sent via email or text message on a weekly basis. Dancers also indicated a tendency to forget to fill out the surveys on occasion. It is possible that the self-report nature of the surveys may have overwhelmed, irritated, or otherwise puzzled dancers, as has already been discussed with respect to the weekly injury reports.

A final limitation was the participants' readiness to change-or openness to new information - that could have impacted their approach towards the instrumentation, the intervention content, and/or the study overall (Prochaska, DiClemente, \& Norcross, 1992). Without directly assessing their attitudes towards sport psychology or performance consulting, it remains unclear whether the dancers' excitement about this study could have influenced their responses. This may be especially important for the days when they did not have contact with the primary researcher. Furthermore, participants knew that their classmates were a part of the study, given the group intervention format. Therefore, it is possible that peer influence played a role in their expressed interest in, or adherence to, the present study. 


\section{Future Recommendations}

Several recommendations can be made as a result of this study. First and foremost, more intervention studies that adopt a quasi-experimental or randomized control trial design should be conducted with a dance population (Noh \& Morris, 2004). Both of these research designs have costs and benefits, but are most likely to allow for further insight into group differences as a result of intervention program content. Thus, the designs may result in best-practice suggestions to the larger dance community. "At the most basic level, certain mental aspects of peak performance are generic and generalize to all types of performers, such as the need to enhance motivation, regulate anxiety, and increase concentration... [But] knowledge of the domainspecific aspects of the performing arts adds greatly to the consultant's repertoire" (Hamilton \& Robson, 2006, p. 254). As Hamilton and Robson (2006) suggest, researchers and applied practitioners who do work in the dance community may find the process both rewarding for themselves and beneficial to participants.

Another recommendation is to utilize additional instrumentation that assesses a wide variety of coping skills within the dance context, with the intent to more fully capture participants' improvements in self-reported psychological skills and abilities. The Athletic Coping Skills Inventory-28 was initially developed for student-athletes who participate in traditional or mainstream sports (Smith, Schutz, Smoll, \& Ptacek, 1995). The present study has shown lower internal consistency on one of the instrument's subscales, and a similar finding with dancers was reported by Estanol, Shepherd, and MacDonald (2013).

Also, from a qualitative perspective, adding a question or two to the focus group discussion may have helped to capture a sense of the participants' self-reported growth, or new skill acquisition. A question about the dancers' expectations of the project or intervention 
program specifically may have produced useful insight regarding their approach to the ACSI28's items. Similarly, a question about their perceived improvement in coping skills over time may have signified positive attitude change. This suggestion somewhat parallels the concept of social support described by Bianco and Eklund (2001), in which perceived, or intended, support may be a more important predictor of athlete wellness than actual, or received, social support.

It is also recommended that intervention-based studies be conducted with the modifications described in the present study's focus group discussion. Changes to time frame, content, length, and perhaps even location (e.g., outside of the typical dance classroom in a more neutral space) could produce different reactions or findings. If a researcher has the means to do so, involving other health and wellness professionals, such as athletic trainers, in the psychoeducation process could be advantageous. Kelman (2000) suggests that psychoeducation programming adopt a "team" approach to include professionals from nutrition, orthopedics, psychology, physical therapy, and medicine. In this way, the dancer can get a holistic view of their health and health habits, and be encouraged to examine how wellness (or lack thereof) may impact their stress level and/or their risk of physical injury. In addition to involving other wellness professionals in the delivery of information, these persons could help to objectively track injury frequency and duration, thus removing subjectivity from dancers' weekly reports.

It is also recommended that researchers continue to determine how to best measure injury within the dance population. This is especially important, given the fact that dancers traditionally may not take time off from dancing unless it is absolutely necessary. This was indeed the case in the present study. Only three dancers reported time lost due to their injury or pain status, and fewer than $20 \%$ of the injury reports included any mention of a modified class, rehearsal, or performance. Some researchers have adopted a "time-loss" definition of injury, stating that it is a 
physical condition "resulting from participation that forces the athlete to interrupt or modify his [or her] usual training plan for at least one training unit," which may be operationally defined as a rehearsal session, performance, or day's worth of classes (Malisoux, Frisch, Urhausen, Seil, \& Theisen, 2013, p. 2896).

This definition appears to be applicable to the dance population, given that Thomas and Tarr (2009) have observed that "dancers tend to define injury as something that stops them from dancing or from moving normally” (p. 51). Nevertheless, Russell (2013) notes that factors including peer or teacher influence, dancer's motivation, pain tolerance, and access to medical care may also contribute to the complexity of defining and then obtaining injury reports. Another way to approach an operational definition of injury may be to quantify dancers' self-initiated treatment strategies, such as taking medication (e.g., Advil) or sitting in an ice bath. Injury might further be defined as a dancer's choice to withdraw from non-dance activities that require physical exertion (e.g., cross-training, socializing with friends). If an athletic trainer or physician is on site, obtaining participants' written permission to view and code medical records could be another approach that capitalizes on collaboration with other wellness professionals.

As is evident from the present study's findings, and the above suggestions, injury within the context of dance remains incredibly challenging to define. As Russell (2013) explains: "one of the difficulties with the current dance literature is its history of multiple definitions for what constitutes an injury and the lack of a standard injury reporting system," and the author further states that the Standard Measure Consensus Initiative of the International Association for Dance Medicine and Science has attempted to address this problem (p. 200).

Additionally, it is recommended that researchers consider adopting at least one instrument to capture some of the overall context and nuances of dance- or dance program- 
specific culture. Utilizing a measure of perfectionism can help researchers to examine adaptive and maladaptive perfectionism among dancers (e.g., Multidimensional Perfectionism Scale; Hewitt \& Flett, 1991; Almost Perfect Scale-Revised; Slaney, Rice, Mobley, Trippi, \& Ashby, 2001). Similarly, a measure of social conformity and/or conformity to the sport ethic (Hughes \& Coakley, 1991) might provide deeper insight as to the cultural norms and expectations of dancers' schools, studios, or peer groups (e.g., the Risk of Pain and Injury Questionnaire; Nixon, 1996; Walk \& Wiersma, 2005).

Furthermore, it is recommended that any intervention program that depends upon participants' individual, self-initiated practice of skills or strategies employ a more thorough reminder system. As expressed in the focus group discussion, participants occasionally forgot to practice their skills until they were prompted by an email or text message to submit their adherence journal report. Researchers, applied practitioners, and dance educators are encouraged to develop and implement a reminder system that is consistent, dependable, and tailored to the dancers' communication preferences.

Based on the information gathered via the Dance Experiences Survey in the present study, it is also recommended that sport psychology practitioners and dance educators find ways to help dancers identify and manage their stressors. These stressors may be inherent to dance culture, or be positive or negative demands that dancers bring with them into the dance studio from other places, such as the school or home environment. Within the present sample, all but one dance student identified time management as a demand that had a current impact on their dancing, and perhaps more generally, their college experience. While some students rated this stressor as positive (presumably stating that they felt capable of managing their time), almost half of the dancers rated its impact as negative. This is one area where psychoeducational 
sessions about proactive planning, scheduling, and general life skills development could be beneficial to dancers. This may be especially true for dance students who do not necessarily see themselves pursuing a professional dance career, but likely see themselves teaching dance to others or making dance a part of their self-care routine while pursuing other job options.

Additionally, given the large number of negative ratings that "physical characteristics" received via the Dance Experiences Survey, it is important that practitioners and educators be mindful of the historical and current pressures that dancers face with regards to body type, body size, and body acceptance. This may be particularly true for those focusing their studies on ballet, which often calls for a particular physique and aesthetic that some dancers may attempt to achieve through self-directed means (e.g., excessive dieting, substance use/abuse, over exercise).

Dancers may benefit from cognitive-behavioral interventions that address negative selftalk about their bodies. Still further, dancers may benefit from intervention content that stems from a mindfulness-based and/or Acceptance and Commitment Therapy (ACT) approach. Previous pilot research has shown improvements in body dissatisfaction and eating attitudes following a brief ACT workshop tailored to women (Pearson, Follette, \& Hayes, 2012). Whether dancers go on to have professional careers or not, preventing eating disorders and increasing self-esteem would seem to be important to their overall development.

Furthermore, it is recommended that researchers continue to examine the effect that intervention programs have on other health-related behaviors and outcomes for dancers, such as sleep, social support, and passion. From both a life skills and injury prevention perspective, it is possible that "good sleep quality, social support, and the use of problem-focused coping strategies...may buffer the effects of stress and protect dancers from sustaining injuries" (Rip, Fortin, \& Vallerand, 2006, p. 14). Additionally, sport psychology consultants and dance 
educators may be in the unique position to help dancers recognize the difference between obsessive and harmonious passion and move toward a healthier mindset. Harmonious passion occurs when one freely accepts dance as a part of their identity or an important part of their life, but leaves room for other identities and pursuits as well; their self-esteem and/or social approval are not contingent upon their involvement or success in dance (Rip, Fortin, \& Vallerand, 2006). By helping dancers to make the choice to train and perform within the cultural environs without feeling controlled by them, consultants and educators can promote increased autonomy, and address some of the outdated ideas about what it means to be a "good" or "dedicated" dancer.

Lastly, it is recommended that more mixed-method studies be conducted. Approaches that utilize mixed-methods can help scholars and practitioners to better understand college dance students' attitudes towards a number of wellness topics. These topics include, but are not limited to, sport psychology or performance consulting, stress management or psychological skills training programming, dancing through injury, and reporting injuries. Additionally, qualitative studies may capture dancers' attitudes towards participation in psychological research, and their openness to sharing personal experiences with the injury prevention and rehabilitation processes. Should researchers obtain additional information on these topics, psychological skills training and research methodology may be enriched and, as a result, greatly benefit the dance community. As a consequence, more psychometrically-sound instruments may be developed and tested with this population, which is in many ways unique from other mainstream sports and art forms.

\section{Implications for Practice}

The present study's findings provide many concrete suggestions for scholars and practitioners looking to conduct applied research within the dance population. Based on feedback 
expressed during the focus group discussion, psychological skills training programs should include activities and content that engage dancers in an active way. Participants in the current study indicated that the more "passive" forms of relaxation were sometimes difficult to complete. It is therefore suggested that dancers be given several options for practicing skills like mindfulness, relaxation, and imagery. Instead of sitting, perhaps they would choose to do a walking meditation to promote mindfulness. Similarly, while taking themselves through relaxation or imagery exercises, dancers may choose to slowly stretch on the floor or at the barre.

Additionally, intervention program content should be easily applicable to auditions and other evaluative situations, as indicated by participants in present study's focus group discussion. Imagery scripts with a focus on auditions, exams, or pre-performance scenarios should be provided so that participants are able to select the imagery experience that best meets their needs or preferences at the time of the program's delivery. Additionally, dancers should be encouraged to develop their own imagery scripts based on their current stressors related to academic or dance-specific pursuits.

Psychoeducational, or intervention-based, programming should be developed with the dancers' current schedules in mind. Specifically, their ability to focus and/or relax may be influenced by their classes, time of day, and academic or performance demands. As noted earlier, a needs-assessment or survey of activity/skill preferences should be utilized prior to the intervention program delivery. Equipped with more information during the pre-intervention process, the researcher or practitioner can better tailor the program's content and logistics to the specific sample or group.

For instance, dancers in the present study implied that at times they felt compelled to engage in physical practice or rehearsal for upcoming performances rather than to take time to 
learn and refine their mental skills. Based on the data collected, dancers seemed to find time to practice deep breathing, perhaps due to its simplicity or direct application to physical activity. They were less likely to practice mindfulness and progressive muscle relaxation, potentially due to competing time demands and/or the undesired state of being relaxed such that there is a risk of falling asleep or, in contrast, feeling more overwhelmed. In the future, researchers should address barriers to participation up-front, and emphasize the applicability of the program to dancers' current goals and stressors.

It should also be noted that most dancers in the present study reported practicing the skills and strategies that they had learned each week for 30 minutes or less. Therefore, it is recommended that those person(s) delivering programming consider choosing activities and exercises that can be replicated in under a half-hour's time. Interestingly, all of the skills and strategies introduced in the present study's intervention program took less than 30 minutes to execute once they were introduced. However, it appears that dancers perceived these exercises to still be too time-demanding. Researchers and practitioners may need to emphasize the dancer's freedom to modify exercises and strategies to fit their personal preferences. For instance, dancers may choose to spread out their practice over the course of a day, rather than sitting down for a consecutive 20 minutes.

As previously mentioned, the timing of the program may be important to consider. Based on the current study's findings, it is recommended that practitioners offer psychological skills programming at the start of an academic year, semester, or quarter. In this way, dancers can have more time to learn and then practice skills and strategies that they may choose to implement as stress (e.g., academic, social, vocational) may increase. Dancers in the present study indicated a desire to meet more frequently than once per week. The focus group discussion resulted in the 
recommendation of two meetings per week, over a period of approximately 10 to 16 weeks. Should a researcher or practitioner have access to a dance program for a longer period of time, a developmental model might be adopted, such that a skill like deep breathing is introduced and mastered before a dancer learns and practices mindfulness-based strategies.

While researchers have stated that a period of five to 15 sessions is an acceptable time frame for brief therapy and mental health service delivery in the university setting (Pinkerton \& Rockwell, 1994), this guideline may not be applicable to behavior or attitude changes in a college dance context. Interestingly, Noh, Morris and Andersen's (2003) call for more dancespecific interventions fails to recommend an ideal time-frame for the delivery of psychological skills programming. What the researchers do emphasize is the importance of dancers' investment in and ownership of the intervention content. They state that programs similar in content to that of the present study's are likely to be most effective when dancers "find time in their busy schedules to systematically apply these techniques and programs" (p. 38).

Lastly, a best-practice suggestion is to hold these psychoeducational or intervention program sessions in a different location than where dancers typically have their classes and rehearsals. By finding an alternate space, dancers may be able to more fully focus on their reason for attending and not be tempted to want to dance in the space. Additionally, although not stated explicitly in the focus group discussion, dancers may find that a dance studio is not an ideal space to learn and practice relaxation strategies. The sometimes-noisy nearby studios, classroom temperature and/or airflow, and hard floors may not be suitable to participants' relaxation needs or preferences. Practitioners might consider finding a multipurpose space that is easy for dancers to locate on campus but at the same time is conducive to uninterrupted learning experiences. 


\section{Concluding Thoughts}

The present study sought to examine the impact of a six-week psychological skills training program on a sample of college dancers' self-reported coping skills, pain appraisals, and injuries. Although many of the findings were non-significant, the quantitative and qualitative results on the whole can provide several recommendations and best-practice guidelines for future research and applied sport/performance psychology programming. "All of the literature taken together indicates...that the reduction of injury incidence in dancers is a worthwhile, if elusive, target” (Russell, 2013, p. 201). While operationally defining and measuring behavior change and injuries among dancers may be both a difficult and still-developing task, it is clear that more research is needed. Dancers at all levels seek to manage physical, psychosocial, and environmental demands in pursuit of their goals. These goals may include performance satisfaction, socialization, technical mastery, or fitness. It is important that sport psychology consultants and other helping professionals use their knowledge and skills to push the literature forward, and to aid in psychological skills education for all who love to dance. 


\section{References}

Ahlgren-Bedics, R., \& Monda, S. (2009). Life skills for collegiate student-athletes: Defining the need and model practices. In E. F. Etzel (Ed.), Counseling and psychological services for college student-athletes (pp. 113-142). Morgantown, WV: Fitness Information Technology.

Allen, N., Nevill, A., Brooks, J., Koutedakis, Y., \& Wyon, M. (2012). Ballet injuries: Injury incidence and severity over 1 year. Journal of Orthopaedic \& Sports Physical Therapy, 42(9), 781-790.

American Psychological Association. (2005). Men and women: No big difference. Retrieved from http://www.apa.org/research/action/difference.aspx

Anderson, R., \& Hanrahan, S. J. (2008). Dancing in pain: Pain appraisal and coping in dancers. Journal of Dance Medicine \& Science, 12(1), 9-16.

Andersen, M. B., \& Williams, J. M. (1988). A model of stress and athletic injury: Prediction and prevention. Journal of Sport \& Exercise Psychology, 10, 294-306.

Bartley, E. J., \& Fillingim, R. B. (2013). Sex differences in pain: A brief review of clinical and experimental findings. British Journal of Anaesthesia, 111(1), 52-58.

Bauman, P. A., Gallagher, S. P., \& Hamilton, W. G. (1996). Common foot, ankle, and knee problems in professional dancers. Orthopaedic Physical Therapy Clinics of North America, 5(4), 497-513.

Bell, R. J., Skinner, C. H., \& Fisher, L. A. (2009). Decreasing putting yips in accomplished golfers via solution-focused guided imagery: A single-subject research design. The Journal of Applied Sport Psychology, 21(1), 1-14. 
Bianco, T., \& Eklund, R. C. (2001). Conceptual considerations for social support research in sport and exercise settings: The case of sport injury. Journal of Sport \& Exercise Psychology, 23, 85-107.

Brewer, B. W., Van Raalte, J. L., \& Linder, D. E. (1993). Athletic identity: Hercules muscles or Achilles heel? International Journal of Sport Psychology, 24(2), 237-254.

Carpenter, G., \& Patil, M. (2017). Gender differences in pain. In M. A. Huntoon (Ed.), Pain Medicine: Board Review. New York: Oxford University Press.

Carver, C. S., Scheier, M. F., \& Weintraub, J. K. (1989). Assessing coping strategies: A theoretically based approach. Journal of Personality and Social Psychology, 56(2), 267283.

Cohen, J. (1988). Statistical power analysis for the behavioral sciences $\left(2^{\text {nd }}\right.$ ed.). Lawrence Erlbaum Associates.

Coppel, D. B. (1980). Relationship of perceived social support and self-efficacy to major and minor stresses (Doctoral dissertation), University of Washington, Seattle.

Creswell, J. W. (2007). Qualitative inquiry and research design: Choosing among five approaches $\left(2^{\text {nd }}\right.$ ed.). Thousand Oaks, CA: Sage Publications.

Crocker, P. R. E., Kowalski, K. C., \& Graham, T. R. (1998). Measurement of coping strategies in sport. Morgantown, EV: Fitness Information Technology.

Danish, S. J., Petitpas, A. J., \& Hale, B. D. (1993). Life development intervention for athletes. The Counseling Psychologist, 21(3), 352-385.

Dick, R., Agel, J., \& Marshall, S. W. (2007). National Collegiate Athletic Association injury surveillance system commentaries: Introduction and methods. Journal of Athletic Training, 42(2), 173-182. 
Dick, R. W., Berning, J. R., Dawson, W., Ginsburg, R. D., Miller, C., \& Shybut, G. T. (2013). Athletes and the arts: The role of sports medicine in the performing arts. Current Sports Medicine Reports, 12(6), 397-403.

Ekegren, C. L., Quested, R., \& Brodrick, A., (2013). Injuries in pre-professional dancers: Incidence, characteristics, and consequences. Journal of Science and Medicine in Sport, $17,271-275$.

Encarnacion, M. L. G., Meyers, M. C., Ryan, N. D., \& Pease, D. G. (2000). Pain coping styles of ballet performers. Journal of Sport Behavior, 23, 20-32.

Estanol, E., Shepherd, C., \& MacDonald, T. (2013). Mental skills as protective attributes against eating disorder risk in dancers. Journal of Applied Sport Psychology, 25, 209-222.

Faul, F., Erdfelder, E., Lang, A.-G., \& Buchner, A. (2007). G*Power 3: A flexible statistical power analysis program for the social, behavioral, and biomedical sciences. Behavior Research Methods, 39, 175-191.

Gay, L. R., \& Airasian, P. W. (2003). Educational research: Competencies for analysis and Applications. Upper Saddle River, NJ: Merrill/Prentice Hall.

Grove, J. R., Main, L. C., \& Sharp, L. (2013). Stressors, recovery processes, and manifestations of training distress in dance. Journal of Dance Medicine \& Science, 17, 70-78.

Hall, H. K., \& Hill, A. P. (2012). Perfectionism, dysfunctional achievement striving and burnout in aspiring athletes: The motivational implications for performing artists. Theatre, Dance and Performance Training, 3(2), 216-228.

Hamilton, L., \& Robson, B. (2006). Performing arts consultation: Developing expertise in this domain. Professional Psychology: Research and Practice, 37(3), 254-259. 
Harlow, L. L. (2014). The essence of multivariate thinking: Basic themes and methods ( $2^{\text {nd }}$ ed.). New York: Routledge.

Hays, K. F. (2002). The enhancement of performance excellence among performing artists. Journal of Applied Sport Psychology, 14(4), 299-312.

Hewitt, P. L., \& Flett, G. L. (1991). Perfectionism in the self and social contexts: Conceptualization, assessment, and association with psychopathology. Journal of Personality and Social Psychology, 60, 456-470.

Hughes, R., \& Coackley, J. (1991). Positive deviance among athletes: The implications of overconformity to the sport ethic. Sociology of Sport Journal, 8(4), 307-325.

Johnson, U. (2000). Short-term psychological intervention: A study of long-term-injured competitive athletes. Journal of Sport Rehabilitation, 9, 207-218.

Johnson, U., Ekegren, J., \& Andersen, M.B. (2005). Injury prevention in Sweden: Helping soccer players at risk. Journal of Sport and Exercise Psychology, 1, 32-38.

Kelman, B. B. (2000). Occupational hazards in female ballet dancers. AAOHN Journal, 48(9), 430-434.

Kerr, G., \& Goss, J. (1996). The effects of a stress management program on injuries and stress levels. Journal of Applied Sport Psychology, 8, 109-117.

Kerr, G., Krasnow, D., \& Mainwaring, L. (1992). The nature of dance injuries. Medical Problems of Performing Artists, 7, 25-29.

Klockare, E. (2013). Evaluation of the dancers' use of psychological skills training techniques and possible effects of the program (Master's thesis document), The Swedish School of Sport and Health Sciences, Sweden. 
Knapp, T. R., \& Schafer, W. D. (2009). From gain score t to ANCOVA F (and vice versa). Practical Assessment, Research \& Evaluation, 14(6). Available online: http://pareonline.net/getvn.asp?v=14\&n=6

Krasnow, D. H. (2005, December). Sustaining the dance artist: Barriers to communication between educators, artists and researchers. Dance Rebooted: Initializing the grid. Ausdance National, Australia.

Krasnow, D., \& Mainwaring, L. (1990). Dance experiences survey. Retrieved January 2016 through personal communication with the survey developers.

Leppma, M. (2012). Loving-kindness meditation and counseling. Journal of Mental Health Counseling, 34(3), 197-205.

Lubker, J. R., Visek, A. J., Watson, J. C., \& Singpurwalla, D. (2012). Athletes' preferred characteristic and qualifications of sport psychology practitioners: A consumer market analysis. Journal of Applied Sport Psychology, 24(4), 465-480.

Maddison, R., \& Prapavessis, H. (2005). A psychological approach to the prediction and prevention of athletic injury. Journal of Sport \& Exercise Psychology, 27, 289-310.

Mainwaring, L. M., Krasnow, D., \& Kerr, G. (2001). And the dance goes on: Psychological impact of injury. Journal of Dance Medicine \& Science, 5(4), 105-115.

Malisoux, L., Frisch, A., Urhausen, A., Seil, R., \& Theisen, D. (2013). Injury incidence in a sports school during a 3-year follow-up. Knee Surgery, Sports Traumatology, Arthroscopy, 21(12), 2895-2900.

Markula, P. (2012, November 30). Feeling pain: Does exercise need to hurt?. Psychology Today. Retrieved from https://www.psychologytoday.com/blog/fit-femininity/201211/feelingpain 
McCambridge, J., Witton, J., \& Elbourne, D. R. (2014). Systematic review of the Hawthorne effect: New concepts are needed to study research participant effects. Journal of Clinical Epidemiology, 67, 267-277.

Meichenbaum, D.H. (1993). Stress inoculation training: A twenty year update. In R.L. Woolfolk, \& P.M. Lehrer (Eds.), Principles and practice of stress management ( $2^{\text {nd }}$ ed.) (pp.373406). New York: Guilford.

Melzack, R. (1987). The McGill Pain Questionnaire: From description to measurement. Anesthesiology, 103, 199-202.

Miller, G. A., \& Chapman, J. P. (2001). Misunderstanding analysis of covariance. Journal of Abnormal Psychology, 110(1), 40-48.

Molnar, M., \& Karin, J. (2017). The complexities of dancers' pain. Journal of Dance Medicine \& Science, 21(1), 3-4.

Mummery, K., Schofield, G., \& Perry, C. (2004). Bouncing back: The role of coping style, social support and self-concept in resilience of sport performance. Athletic Insight, Retrieved from http://www.athleticinsight.com/Vol6Iss3/BouncingBack.htm.

Nixon, H. (1996). The relationship of friendship networks, sports experiences and gender to expressed pain thresholds. Sociology of Sport Journal, 13, 76-86.

Noh, Y., \& Morris, T. (2004). Designing research-based interventions of the prevention of injury in dance. Medical Problems of Performing Artists, 19(2), 82-89.

Noh, Y., Morris, T., \& Andersen, M. B. (2002). Sources of stress and coping strategies of Korean ballet dancers. Annual conference of the Association for the Advancement of Applied Sport Psychology, Tucson, AZ. 
Noh, Y., Morris, T., \& Andersen, M. B. (2005). Psychosocial factors and ballet injuries. International Journal of Sport and Exercise Psychology, 3, 7-25.

Noh, Y., Morris, T., \& Andersen, M. B. (2007). Psychological intervention programs for reduction if injury in ballet dancers. Research in Sports Medicine, 15, 13-32.

Nordin-Bates, S. M., Walker, I. J., Baker, J., Garner, J., Hardy, C., Irvine, S., Jola, C., Laws, H., \& Blevins, P. (2011). Injury, imagery, and self-esteem in dance: Healthy minds in injured bodies? Journal of Dance Medicine \& Science, 15(2), 76-85.

Nordin-Bates, S. M., Quested, E., Walker, I. J., \& Redding, E. (2012). Climate change in the dance studio: Findings from the UK centres for advanced training. Sport, Exercise, and Performance Psychology, 1(1), 3-16.

Omar-Fauzee, M., Daud, W., Abdullah, R. \& Rashid, S. (2009). The effectiveness of imagery and coping strategies in sport performance. European Journal of Social Sciences, 9(1), 97-108.

Patton, M. Q. (2002). Qualitative research \& evaluation methods $\left(3^{\text {rd }}\right.$ Ed.). Thousand Oaks, CA: Sage Publications.

Pearson, A. N., Follette, V. M., \& Hayes, S. C. (2012). A pilot study of Acceptance and Commitment Therapy as a workshop intervention for body dissatisfaction and disordered eating attitudes. Cognitive and Behavioral Practice, 19(1), 181-197.

Perna, F. M., Antoni, M. H., Baum, A., Gordon, P., \& Schneiderman, N. (2003). Cognitive behavioral stress management effects on injury and illness among competitive athletes: A randomized clinical trial. Annals of Behavioral Medicine, 25(1), 66-73.

Prochaska, J. O., DiClemente, C. C., \& Norcross, J. C. (1992). In search of how people change: applications to the addictive behaviors. American Psychologist, 47, 1102-1114. 
Rip, B., Fortin, S., \& Vallerand, R. (2006). The relationship between passion and injury in dance students. Journal of Dance Medicine \& Science, 10(1), 14-20.

Rivers, A. (2014, December 1). When onstage nightmares come true. Dance Magazine. Retrieved from http://dancemagazine.com/insidedm/when_onstage_nightmares_ come_true/

Rosenbaum, M. (1980). A schedule for assessing self-control behaviors: Preliminary findings. Behavior Therapy, 11(1), 109-121.

Russell, J. (2013). Preventing dance injuries: Current perspectives. Open Access Journal of Sports Medicine, 4, 199-210.

Shah, S. (2008). Caring for the dancer: Special considerations for the performer and troupe. Current Sports Medicine Report, 7(3), 128-132.

Slaney, R. B., Rice, K. G., Mobley, M., Trippi, J., \& Ashby, J. S. (2001). The revised Almost Perfection Scale. Measurement and Evaluation in Counseling and Development, 34, 130145.

Smith, R. E., Ptacek, J. T., \& Patterson, E. (2000). Moderator effects of cognitive and somatic trait anxiety on the relation between life stress and physical injuries. Anxiety, Stress, \& Coping, 13(3), 269-288.

Smith, R.E., Smoll, F.L., \& Schutz, R.W. (1990). Measurement and correlates of sport-specific cognitive and somatic trait anxiety: The Sport Anxiety Scale. Anxiety Research, 2, $263-$ 280.

Smith, R. E., Schutz, R. W., Smoll, F. L., \& Ptacek, J. T. (1995). Development and validation of a multidimensional measure of sport-specific psychological skills: The Athletic Coping Skills Inventory-28. Journal of Sport \& Exercise Psychology, 17(4), 379-398. 
Tait, R. C., Chibnall, J. T., \& Krause, S. (1990). The Pain Disability Index: Psychometric Properties. Pain, 40(2), 171-182.

Thomas, H., \& Tarr, J. (2009). Dancer's perceptions of pain and injury: Positive and negative effects. Journal of Dance Medicine \& Science, 13(2), 51-59.

Turner, B. S., \& Wainwright, S. P. (2003). Corps de ballet: The case of the injured ballet dancer. Sociology of Health \& Illness, 25(4), 269-288.

Unruh, A. M., \& Ritchie, J. A. (1998). Development of the Pain Appraisal Inventory: Psychometric properties. Pain Research and Management, 3, 105-110.

van Staden, A., Myburgh, C. P., \& Poggenpoel, M. (2009). A psycho-educational model to enhance the self-development and mental health of classical dancers. Journal of Dance Medicine \& Science, 13(1), 20-28.

Wainwright, S. P., Williams, C., \& Turner, B. S. (2005). Fractured identities: Injury and the balletic body. Health, 9(1), 49-66.

Walk, S., \& Wiersma, L. (2005). Construct validity of the Risk, Pain and Injury Questionnaire. Sociology of Sport Journal, 22, 443-446.

Watson, E. C. (2013). Examining health correlates in an elite professional ballet company (Unpublished doctoral dissertation). The Adler School of Professional Psychology, Chicago, IL.

Weinberg, R. S., \& Gould, D. (2015). Foundations of sport and exercise psychology (6 ${ }^{\text {th }}$ ed.). Champaign, IL: Human Kinetics.

Williams, J. M., \& Andersen, M. B. (1998). Psychosocial antecedents of sport injury: Review and critique of the stress and injury model. Journal of Applied Sport Psychology, 10(1), $5-25$. 
Wozny, N. (2016, August 31). When experts cringe. Dance Magazine. Retrieved from http://dancemagazine.com/inside-dm/magazine/when-experts-cringe/

Yatabe, K., Yui, N., Kasuya, S., Fujiya, H., Tateishi, K., Terawaki, F., Yoshida, A., Yoshioka, H., Terauchi, K., Miyano, H., Oyama, T., \& Musha, H. (2014). Anxiety and mood among ballet dancers: A pilot study on effects of a medical approach involving periodic intervention. Annals of Sports Medicine and Research, 1(1), 1001-1009. 
Table 1

Basic descriptive information for participants presented by group condition

\begin{tabular}{lccccccc}
\hline & \multicolumn{3}{c}{ Control } & & \multicolumn{3}{c}{ Treatment } \\
\cline { 2 - 4 } \cline { 6 - 8 } & $N$ & $M$ & $S D$ & & $M$ & $S D$ \\
\hline Age & 15 & 18.60 & 0.91 & & 15 & 20.93 & 0.80 \\
Years of Dancing & 15 & 11.40 & 4.14 & & 15 & 15.20 & 4.14 \\
Hours per Week & 15 & 18.33 & 3.58 & & 15 & 17.73 & 4.69 \\
Week 1 ACSI-28 & 15 & 52.00 & 8.26 & & 15 & 45.67 & 10.42 \\
Week 6 ACSI-28 & 14 & 50.14 & 8.11 & & 15 & 47.40 & 12.73 \\
Week 1 PAI & 15 & 38.47 & 15.84 & & 14 & 39.43 & 14.77 \\
Week 6 PAI & 14 & 36.14 & 16.27 & & 15 & 43.07 & 11.49 \\
\hline
\end{tabular}

Note: The ACSI-28 is the Athletic Coping Skills Inventory-28, and the PAI is the Pain Appraisal Inventory. These instruments were administered in-person during week one and week six of the study. 


\section{Table 2}

Internal consistency coefficients for the Athletic Coping Skills Inventory-28

\begin{tabular}{lcc}
\hline & Present Study & Smith et al. (1995) \\
\hline Coping with Adversity & 0.71 & 0.66 \\
Coachability & 0.71 & 0.72 \\
Concentration & 0.66 & 0.62 \\
Confidence \& Achievement Motivation & 0.51 & 0.66 \\
Goal Setting \& Mental Preparation & 0.68 & 0.71 \\
Peaking under Pressure & 0.86 & 0.78 \\
Freedom from Worry & 0.81 & 0.76 \\
Total & 0.73 & 0.86 \\
\hline
\end{tabular}

Note: Cronbach's alphas were observed in the present study at week one, before the start of the intervention program for treatment group participants. Cronbach's alphas reported for Smith, Schutz, Smoll, and Ptacek (1995) were taken from Table 3 of their published article. 
Table 3

Test-retest correlations for the Pain Appraisal Inventory and its subscales

\begin{tabular}{lcccccc}
\hline & 1. & 2. & 3. & 4. & 5. & 6. \\
\hline 1. Total 1 & -- & & & & & \\
2. Total 2 & $.399^{* *}$ & -- & & & & \\
3. Threat 1 & $.742^{* *}$ & .163 & -- & & & \\
4. Threat 2 & .176 & $.771^{* *}$ & .111 & -- & & \\
5. Challenge 1 & $.792^{* *}$ & $.430^{*}$ & .178 & .154 & -- & \\
6. Challenge 2 & $.459^{*}$ & $.847^{* *}$ & .154 & .315 & $.527^{* *}$ & -- \\
\hline
\end{tabular}

Note: Total 1 = participants' total scores on the Pain Appraisal Inventory for the first week of the study; Total 2 = participants' total scores on the Pain Appraisal Inventory for the final week of the study; Threat 1 = participants' scores on the threat subscale for the first week of the study; Threat 2 = participants' scores on the threat subscale for the final week of the study; Challenge 1 = participants' scores on the challenge subscale for the first week of the study; Challenge 2 = participants' scores on the challenge subscale for the final week of the study. $* \mathrm{p}<.05$, two-tailed $* * \mathrm{p}<.01$, two-tailed 


\section{Table 4}

Test-retest correlations for the Pain Appraisal Inventory and its subscales for participants in the control group

\begin{tabular}{lcccccc}
\hline & 1. & 2. & 3. & 4. & 5. & 6. \\
\hline 1. Total 1 & -- & & & & & \\
2. Total 2 & .229 & -- & & & & \\
3. Threat 1 & $.679^{* *}$ & -.002 & -- & & & \\
4. Threat 2 & -.192 & $.718^{* *}$ & -.196 & -- & & \\
5. Challenge 1 & $.844^{* *}$ & .305 & .179 & -.106 & -- & \\
6. Challenge 2 & .454 & $.867^{* *}$ & .138 & .277 & .497 & -- \\
\hline
\end{tabular}

Note: Total $1=$ total scores on the Pain Appraisal Inventory for the first week of the study for control group; Total 2 = total scores on the Pain Appraisal Inventory for the final week of the study for control group; Threat $1=$ scores on the threat subscale for the first week of the study for control group; Threat $2=$ scores on the threat subscale for the final week of the study for control group; Challenge $1=$ scores on the challenge subscale for the first week of the study for control group; Challenge 2 = scores on the challenge subscale for the final week of the study for control group.

$* \mathrm{p}<.05$, two-tailed $* * \mathrm{p}<.01$, two-tailed 


\section{Table 5}

Test-retest correlations for the Pain Appraisal Inventory and its subscales for participants in the treatment group

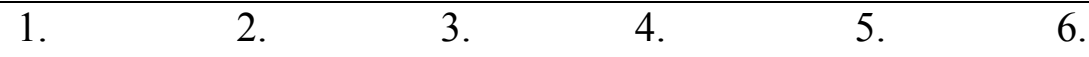

\begin{tabular}{|c|c|c|c|c|c|}
\hline 1. Total 1 & -- & & & & \\
\hline 2. Total 2 & $.702 * *$ & -- & & & \\
\hline 3. Threat 1 & $.827 * *$ & .318 & -- & & \\
\hline 4. Threat 2 & $.709 * *$ & $.819 * *$ & .336 & -- & \\
\hline 5. Challenge 1 & $.749 * *$ & $.835 * *$ & .247 & $.825^{* *}$ & -- \\
\hline 6. Challenge 2 & $.541 *$ & $.856^{* *}$ & .228 & .404 & $.664 * *$ \\
\hline
\end{tabular}

Note: Total $1=$ total scores on the Pain Appraisal Inventory for the first week of the study for treatment group; Total $2=$ total scores on the Pain Appraisal Inventory for the final week of the study for treatment group; Threat $1=$ scores on the threat subscale for the first week of the study for treatment group; Threat $2=$ scores on the threat subscale for the final week of the study for treatment group; Challenge $1=$ scores on the challenge subscale for the first week of the study for treatment group; Challenge $2=$ scores on the challenge subscale for the final week of the study for treatment group.

$* \mathrm{p}<.05$, two-tailed

$* * \mathrm{p}<.01$, two-tailed 
Table 6

Analysis of covariance for the Athletic Coping Skills Inventory-28 to examine a possible difference in mean scores between the control group and the treatment group (i.e., condition)

\begin{tabular}{lccccccc}
\hline & $\mathrm{SS}$ & $\mathrm{df}$ & $\mathrm{MS}$ & $F$ & $p$ & $\eta^{2}$ & power \\
\hline Intercept & 47.442 & 1 & 47.442 & 0.940 & 0.341 & 0.035 & .154 \\
Covariate & 1813.436 & 1 & 1813.436 & 35.940 & 0.000 & 0.580 & 1.000 \\
Condition & 50.665 & 1 & 50.665 & 1.004 & 0.326 & 0.037 & .162 \\
Error & 1311.878 & 26 & 50.457 & -- & -- & -- & -- \\
Total & 72027.00 & 29 & -- & -- & -- & -- & -- \\
\hline
\end{tabular}

Note: $\mathrm{SS}=$ Sum of Squares; $\mathrm{df}=$ degrees of freedom; $\mathrm{MS}=$ Mean Square; $F=$ F-ratio; $p=$ significance level; $\eta^{2}=$ eta squared; power = observed power. 
Table 7

Analysis of covariance for the Pain Appraisal Inventory to examine a possible difference in mean scores between the control group and the treatment group (i.e., condition)

\begin{tabular}{lccccccc}
\hline & $\mathrm{SS}$ & $\mathrm{df}$ & $\mathrm{MS}$ & $F$ & $p$ & $\eta^{2}$ & power \\
\hline Intercept & 1890.838 & 1 & 1890.838 & 11.212 & 0.003 & 0.310 & .896 \\
Covariate & 864.658 & 1 & 864.658 & 5.127 & 0.032 & 0.170 & .586 \\
Condition & 262.101 & 1 & 262.101 & 1.554 & 0.224 & 0.059 & .224 \\
Error & 4215.985 & 25 & 168.639 & -- & -- & -- & -- \\
Total & 48149.00 & 28 & -- & -- & -- & -- & -- \\
\hline
\end{tabular}

Note: $\mathrm{SS}=\mathrm{Sum}$ of Squares; $\mathrm{df}=$ degrees of freedom; MS = Mean Square; $F=$ F-ratio; $p=$ significance level; $\eta^{2}=$ eta squared; power $=$ observed power. 
Table 8

Weekly injury status reports by group condition

\begin{tabular}{lccccccc}
\hline & \multicolumn{3}{c}{ Control } & & \multicolumn{3}{c}{ Treatment } \\
\cline { 2 - 4 } \cline { 6 - 8 } & $n$ & Yes & No & & & Yes & No \\
\hline Week 1 & 15 & 4 & 11 & & 15 & 9 & 6 \\
Week 2 & 7 & 2 & 5 & & 11 & 6 & 5 \\
Week 3 & 6 & 2 & 4 & & 11 & 5 & 6 \\
Week 4 & 8 & 2 & 6 & & 11 & 3 & 8 \\
Week 5 & 6 & 3 & 3 & & 10 & 3 & 7 \\
Week 6 & 14 & 6 & 8 & & 15 & 9 & 6 \\
\hline
\end{tabular}

Note: "Yes" = participant indicated having one or more physical injuries in that week; "No" = participant indicated not having one or more physical injuries in that week. 


\section{Table 9}

Regions or areas of the body affected by injury across all study participants

\begin{tabular}{lc}
\hline & Number of Reported Instances \\
\hline Head/Face & 0 \\
Neck/Cervical Spine & 2 \\
Shoulder/Clavicular & 4 \\
Upper Arm & 0 \\
Sternum/Ribs/Upper Back & 2 \\
Elbow/Forearm & 1 \\
Abdomen & 0 \\
Lower Back/Pelvis/Sacrum & 10 \\
Hip/Groin & 11 \\
Wrist & 0 \\
Hand/Finger/Thumb & 1 \\
Thigh & 0 \\
Knee & 11 \\
Lower Leg/Achilles Tendon & 22 \\
Ankle & 20 \\
Foot/Toe & 16 \\
\hline
\end{tabular}

Note: This table presents the areas or regions of the body that were affected by injury, as reported by this sample of university-level dancers. It should be noted that it is possible that participants reported these affected areas not only when they were experiencing injuries, but also when their bodies were affected by injury-related, or generalized training, pain. 
Table 10

Top ten stressors for all participants as reported via the Dance Experiences Survey

\begin{tabular}{lccccc}
\hline & $N$ & Positive & Neutral & Negative & N/S \\
\hline 1. Managing Time & 29 & 10 & 6 & 13 & 0 \\
2. Physical Characteristics & 28 & 6 & 2 & 20 & 0 \\
3. Support from Others & 27 & 22 & 1 & 4 & 0 \\
4. Performing & 27 & 23 & 2 & 2 & 0 \\
5. Important Upcoming Performance & 27 & 18 & 0 & 9 & 0 \\
6. Motivation & 27 & 12 & 4 & 11 & 0 \\
7. Physical Demands of Training & 26 & 18 & 1 & 7 & 0 \\
8. Psychological Demands of Training & 26 & 9 & 3 & 13 & 1 \\
9. Diet & 26 & 11 & 3 & 12 & 0 \\
10. Unmet Goals & 26 & 4 & 2 & 20 & 0 \\
\hline
\end{tabular}

Note: $N=$ Number of dance students reporting this current stressor (out of 30 dance students); Positive $=$ Impact of this stressor was rated positively; Neutral $=$ Impact of this stressor was rated neutral; Negative = Impact of this stressor was rated negatively; N/S = An impact rating was not specified for this stressor. 


\section{Table 11}

Overview of skills practiced as reported via treatment group participants' adherence journals

\section{Number of Reports}

\begin{tabular}{lc}
\hline Deep or Diaphragmatic Breathing & 21 \\
Progressive Muscle Relaxation & 5 \\
Imagery & 24 \\
Self-Talk/Cognitive Restructuring & 27 \\
Mindfulness & 10 \\
Other & 2 \\
None & 1 \\
$\begin{array}{l}\text { Note: These numbers only represent the skills that were reportedly practiced over the course of } \\
\text { the intervention program. Not all participants provided weekly reports via the adherence journal, } \\
\text { and some participants submitted reports for some weeks, but not for other weeks. }\end{array}$
\end{tabular}




\section{Appendix A: Extended Review of Literature}

This chapter is intended to provide a review of the literature that informed the present study. It will begin with an overview of sports injury, and then move into a focused discussion of dance injury and dance culture. The chapter will also introduce the model on which the present study was based, and then cover the use and efficacy of short-term interventions across a variety of settings and populations in athletics and the performing arts. Specifically, psychological skills training programs, stress-inoculation programs, and cognitive-behavioral therapy approaches will be examined as protocols that informed the present study's intervention-based design. Finally, the chapter will conclude with a brief discussion of the potential outgrowths of additional research specifically addressing psychosocial factors related to dance injury.

\section{Sports Injury}

According to the Center for Disease Control (2015), physical injury is the number-one cause of death among persons ages one to 44. In 2010, injury accounted for over five million visits to hospital emergency rooms among persons aged 16 to 25 (CDC, 2015). Each of these non-specified injuries (i.e., occurred across all body regions) had an estimated average medical cost of $\$ 2,334$. The total lifetime cost to treat all of these injuries was an estimated $\$ 13,105,494,000$ (CDC, 2015). Given the lifetime costs, it is imperative to examine the causes of these injuries and to determine instances in which injury prevention programs can be effective.

Injuries as a result of sports participation make up a rather large percentage of all physical injuries. It is estimated that sport-related injuries account for approximately $20 \%$ of all injury-related emergency department visits annually, among persons aged 6 to 19 (Department of Health and Human Services, 2014). Each year, an estimated 12 million persons aged 5 to 22 experiences a sport-related injury; this results in an estimated 20 million missed school days and 
“approximately \$33 billion in health care costs" (Department of Health and Human Services, 2014, p. 3). These large statistics point to the need for preventative measures, including but not limited to increased education about general safety protocols and injury susceptibility within organized and recreational activities such as sports and the performing arts.

Injury is a common occurrence across sports and the performing arts. It is a primary area of focus for many researchers and practitioners due to its severity, complexity, and inevitability (e.g., Goldberg, Moroz, Smith, \& Ganley, 2007). The National Institutes of Health broadly defines "sports injuries" as injuries that most commonly occur during sports or physical activity/exercise. "Some sports injuries result from accidents; others are due to poor training practices, improper equipment, lack of conditioning, or insufficient warm-up and stretching" (NIH, 2014). The NIH also states that, while injuries can occur on any body part or region, the word "injury" in the sports context most commonly refers to injuries sustained to the musculoskeletal system (i.e., muscles, bones, and tissues).

Sports injuries are commonly classified as either "acute" or "chronic" (NIH, 2014). Acute injuries are injuries that occur relatively suddenly when a person is performing a skill or activity (NIH, 2014). Examples of acute injuries include strained muscles and sprained ankles. Chronic injuries, on the other hand, are injuries that arise due to overuse of one or more body parts or regions over time (NIH, 2014). Examples of chronic injuries include stress fractures and tendonitis. Some of the most common sports injuries are: sprains and strains, knee injuries, swollen muscles, Achilles tendon injuries, pain along the shin bone, fractures, and dislocations (NIH, 2014). It is important to note that the incidence and prevalence of common injuries and injury types may vary across persons, depending upon training workload and factors like proper equipment use (DiFiori et al., 2014). 
Athletes are also susceptible to injuries related to overexertion. Overexertion injuries often occur as a result of fatigue, dehydration, hypoglycemia, or a combination of these factors; and while most are preventable, they account for over three million hospital visits a year (Healthline, 2013). As is evident in the magnitude of these statistics, injury is a nation-wide issue that merits further examination both in the research literature and in applied settings.

While the NIH and CDC tend to define injury in broad terms, the research literature tends to use narrower, albeit sometimes conflicting, definitions of injury. For instance, one of these definitions has been coined the "time-loss definition," and is operationalized as: "a physical complaint resulting from sports participation that forces the athlete to interrupt or modify his usual training plan for at least one training unit" (Malisoux, Frisch, Urhausen, Seil, \& Theisen, 2013, p. 2896). A training unit may consist of one practice, rehearsal, work out, or competition, depending on the main activity scheduled for the athlete on a particular day. Furthermore, researchers can categorize injuries by severity, using the number of training or competition days missed: slight (0-3 days), minor (4-7 days), moderate (8-28 days), and major ( $>28$ days) (Malisoux et al., 2013). Some researchers note that defining injury from a time-loss perspective is not inclusive of all injuries, citing the fact that tissue damage and minor bruises could still be described as injuries even if athletes do not miss a practice or game (Goldberg, Moroz, Smith, \& Ganley, 2007). Lastly, yet another definition could be the presence or absence of a doctor's visit, deeming an injury to be recorded when an athlete seeks medical help (Goldberg, Moroz, Smith, \& Ganley, 2007).

Naturally, there are limitations when defining and quantifying injury. For instance, Russell (2013) notes that the "identification of an occurrence as an 'injury' only if it results in time lost from an individual's activity" is a common guideline in epidemiological research (p. 
200); however, as previously state, this definition neglects the injuries sustained by persons who continue to exercise or perform while injured or in pain. Additionally, injury reporting is affected by an individual's motivation, his or her pain tolerance, peer influence, and the larger culture that a coach or instructor creates (Russell, 2013). Access to appropriate medical care is also likely to influence the quality and quantity of injury data that are collected and reported (Russell, 2013).

As a result, it is clear that "there is no single consensus on the definition of a reportable injury" (Goldberg, Moroz, Smith, \& Ganley, 2007, p. 268). Therefore, researchers must operationally define the construct within the scope of their research, while being responsive to the environment, context, and population in which an observed injury occurs. For instance, it is possible that common injuries sustained in soccer are not the same as those sustained in ballet, and thus unique physical and mental subcultures of each training environment will influence injury risk and reporting.

\section{Dance Injury}

Like athletes, researchers suggest that dancers have a high probability of experiencing injury as part of their training and performance lifestyle (Krasnow, Kerr, \& Mainwaring, 1994; Turner \& Wainwright, 2003). According to several studies (e.g., Allen, Nevill, Brooks, Koutedakis, \& Wyon, 2012; Bronner, Ojofeitimi, \& Rose, 2003), there exists a high injury rate among professional and pre-professional dancers, with Krasnow et al. (2004) reporting a rate of up to $97 \%$ of dancers experiencing a physical injury in a one-year period. Similarly, Shah (2008) reported that "up to $95 \%$ of ballerinas and $82 \%$ of modern dancers will have at least one injury" over the course of a year (p. 129).

In a study conducted with pre-professional elite dancers $(N=266)$ aged 15 to 19 , Ekegren, Quested, and Brodrick (2013) found that 76\% of the dancers in their sample 
experienced at least one injury per year. The researchers also reported a critical incidence rate of 1.42 injuries per dancer per year, and an estimated 1.38 injuries per 1,000 hours of dancing. Additionally, they found that chronic injuries were more common than traumatic (or acute) injuries, with stress fractures and injuries to the joints, ankles, and knees to be most common. These injuries are the likely result of constant, meticulous training at a "level of precision... comparable to that of an Olympic gymnast." (Smith, as quoted in Schwarz, 2000). As is further articled by Shah (2008):

Dance is not only a performing art, but a highly rigorous athletic sport. In fact, one study [Nicholas, 1975] concludes that when compared with 61 other athletic sports (including football, rugby, boxing, and hockey), ballet is one of the most physically and mentally demanding... Dance is designed to look aesthetically pleasing, while often performed at extreme ranges of motion. (p. 128)

Dancers are likely to experience injury for a number of reasons. The subsequent section will explore these reasons, highlighting the influence that environmental and psychosocial factors may have on injury susceptibility and pain tolerance within the dance world.

\section{Dance Culture}

It is critical that researchers and practitioners alike recognize the uniqueness of the dance culture, its norms and expectations in order to understand the concept of dance injury. In a written report focused on caring for dancers' health, Shah (2008) claims that dance basics (such as technique, class or performance structure, and flooring) and dancer mentality are among the important factors to consider when explaining how and why injury occurs. To elaborate, Shah (2008) implies that an assessment of injury risk should include a basic understanding of the technique emphasized in the dance class, program or company, given that different dance styles 
and techniques may more commonly subject dancers to different types of injuries. For instance, Solomon and Micheli (1986) reported that dancers who study Graham technique are likely to experience knee injuries, while dancers who study Horton technique are more likely to injure their lower backs.

The class or performance structure also matters, given that the warm-up is the time period in which dancers need to activate their muscles and begin to get their bodies ready for more intense movement. The flooring, ideally, should be shock resistant with some spring, and achieve a balance between being a slippery and sticky surface so as to prevent falls (Shah, 2008). Lastly, a dancer's mentality is an important factor in injury prevention. Dancers are often described as overachievers reaching for perfection, and so they are unlikely to report injuries until the pain negatively interferes with their performance (Shah, 2008).

The necessary blend of artistry and athleticism, the repetitive and habitual nature of dance training, and the culture of pain tolerance must be considered when exploring potential causes of injury (Anderson \& Hanrahan, 2008; Dick et al., 2013). Phrases such as “no pain, no gain” and "the show must go on" (Anderson \& Hanrahan, 2008), which can be heard in rehearsal studios and backstage alcoves, suggest a strong culture of tolerance among dancers. The lack of a true "off season" may contribute to this "pushing through pain" mindset. Dancers often engage in cross-training and additional performances year-round, when other athletes are engaged in periodized training or strictly rest and recovery. Dick et al. (2013) noted that at National Collegiate Athletic Association institutions, where student-athletes are permitted to participate in their sports for a maximum of 20 hours per week, it is not uncommon for music and dance students to be practicing for up to six hours per day. 
Thus, dancers are susceptible to injuries year-round, and most especially injuries that result from "chronically overburdening the body" (Aalten, 2005, p. 56; Anderson \& Hanrahan, 2008). If pain is ignored, what once was as a relatively minor issue can become a much more serious one (Aalten, 2005). Injuries that go untreated or unreported can worsen, and lead to musculoskeletal disorders and chronic pain (Rivera, Alexander, Nehrenz, \& Fields, 2012). Common injuries related to overuse in dance include stress fractures in the foot and/or ankle, tendonitis in the knees, and spondylolysis (back pain) (Children's Hospital Colorado Orthopedics Institute, 2014).

Injury can be particularly threatening to a dancer's self-concept (Wainwright, Williams, \& Turner, 2005). It is generally accepted that a dancer's self-concept is comparable to an athlete's athletic identity (Brewer, Van Raalte, \& Linder, 1993). “The performing artist's body (like a sport athlete) is an essential instrument that is the foundation for performance" (Dick et al., 2013, p. 397). Researchers have suggested that dancers' levels of athletic/performance identity reinforce the "oneness" of their craft, body, purpose, and self-image (Wainwright, Williams, \& Turner, 2005). Unlike a musician, whose instrument may be a flute or violin, a dancer's instrument is the body. Therefore, injury to the body is an injury to the self. Suffering from an injury can potentially impact a dancer's self-concept, career trajectory, and overall wellbeing.

Wainwright and colleagues (2005) conducted an ethnographic study of professional dancers' and ex-dancers' embodiment of injury with a sample of dancers $(N=20)$ from the Royal Ballet in London, England. The study’s first author spent over 40 days conducting fieldwork within this dance company, resulting in personal observations and 20 interviews with various ballet staff members and dancers. In reporting their findings, the researchers highlighted 
the interwoven nature of dancers' identity and career. Although injured, a dancer may choose to "keep going," and thus normalize the pain as a necessary part of striving towards a higher level of artistic and athletic perfection. They may also come to view injury as a loss of opportunities towards personal growth and career trajectory (Wainwright et al., 2005).

Indeed, researchers suggest that dancers push through pain in order to maintain a sense of control over current of future job opportunities (Wainwright et al., 2005). If an injured dancer is unable to perform, then another dancer who is not injured will replace him or her, either temporarily or permanently. As Dr. William Hamilton described in an article for Dance Magazine, dancers cannot perform with extremely visible injuries, given the aesthetic of dance: "It takes a lot longer to get back to that edge... there's no hiding bulky bandages and braces under uniforms; dancers must look pristine, showing off clean lines, muscular backs and legs" (Theys, 2011). This statement illustrates the contrast between dancers and athletes when it comes to returning from injury. A soccer player can play with a taped ankle or headgear. Given the artistic nature of dance, however, these sorts of visible rehabilitative and/or preventative measures are not to be found on the stage.

Another factor to consider when addressing dance injury is the role of physicians. One of the difficulties in dancers' reporting and subsequent recovery from injury is their potential mistrust of the medical system. As suggested by Shah, Weiss, and Burchette (2012), dancers may conclude that physicians do not fully understand the dance culture and the unique pressures and demands on their bodies. In their sample of professional modern dancers $(N=184)$, Shah and colleagues reported that $82 \%$ of dancers experienced at least one injury in the past year, but of those dancers who sought medical consultation, only $47 \%$ sought the advice of a physician; others consulted a physical therapist or chiropractor. Shah (2008) postulates that dancers may not 
seek medical attention in part due to the belief that physicians "do not understand their problems and are too quick to instruct them to stop dancing" (Shah, 2008, p. 128). Dancers may negatively receive the prescription to "stop dancing," given their devotedness to their art form and the real or imagined threat of being replaced. Researchers report that injuries can lead to changes in dancers' attitude, stress, performance (Rivera et al., 2012), satisfaction and, for those who are paid, job security (e.g., Wainwright, Williams, \& Turner, 2005).

As previously stated, the dance culture is one that can be demanding on mind and body, especially with a lack of "off season" and an emphasis on achieving physical and artistic “perfection" through unnatural movements and poses (Anderson \& Hanrahan, 2008). A recent blog post by Julia Erickson (2015), a principal dancer with the Pittsburgh Ballet Theatre, expands upon this perfectionistic striving, with a cautionary tale of its consequences:

Because we can't know how others perceive us_-but we all yearn to be desired, loved, and accepted — we tend to make assumptions about what people think, often skewed toward the negative. This tendency is what I call The Black Swan Effect: the darkness that builds up inside of us as a result of how we think others perceive us... The Black Swan character is the archetype of evil; she's a manipulative schemer whose goal is to sway her victim toward darkness and away from light... [the] insidious force creates self-doubt and fear, stifles creative expression, and is, quite frankly, unhealthy.

Erickson's description of this "black swan effect" relates back to the concept of silently suffering from an injury, be it physical or psychological in nature. A dance studio is often full of mirrors and watchful, artistic eyes. Therefore, to show weakness or insecurity would be a threat to pleasing others and a threat to one's own confidence and hopefulness. Due to the potential for 
dance schools and studios to be full of self-conscious students, it is important to understand the environment in which training and instruction occur, and the climate in which dance classes and rehearsals are being led.

Aesthetically, dance is extremely demanding (Dick et al., 2013; Wyon, 2007). At the root of almost every style of dance is ballet. Classical ballet technique requires the dancer to work to achieve a 180-degree turnout of the feet from the pelvis, take performance risks on stage, demonstrate musicality, and maintain a strong stage presence (Hamilton et al., 1989). These aesthetics, characteristic of many if not all celebrated dance icons, are challenging to maintain over time, given that the body is stretched and held in abnormal positions. Unlike individual and team sports, in which performance can ultimately be quantified (e.g., field goal percentage, fouls committed, or score earned) a dancer's overall performance cannot be objectively measured.

The "perfection" of one's athleticism and artistry is subjective (Dick et al., 2013), and thus two viewers or critics may judge the same dancer's performance differently. It is a dancer's ability to convey story and emotion - in conjunction with her physicality — that earns shouts of "bravo!" or a standing ovation. Yet because this success is subjective, the dancer herself may not accept public praise, remaining her hardest critic (Wainwright, Williams, \& Turner, 2005).

Thus, dancers may go their entire careers without feeling like they have "arrived" at perfection. This constant striving can be motivating, but at the same time, mentally and physically exhausting.

The culture of dance lends itself to a discussion of "mental toughness," a concept often talked about in the realm of collegiate and professional athletics (Butt, Weinberg, \& Culp, 2010; Connaughton, Wadey, Hanton, \& Jones, 2008; Jones, 2002). Wainwright, Williams, and Turner (2005) captured dancers' experiences of pain as part of the training process, and suggested that 
some degree of working through pain is what separates the "weak" from the "strong" in the dance world. As one participant told the researchers: "some people were never going to make it in dance, because they didn't have the guts to come in every day and get on with it. They always had pain and it affected them; whereas some people had pain and it didn't affect them” (p. 59). This equating of pain tolerance with mental toughness is important to recognize as part of a larger dance culture in which physical and psychological pressures are commonly present.

In order to better understand the psychosocial profile of people who pursue careers in dance, Hamilton et al. (1989) investigated personality, stress, coping, and injury patterns in a sample of professional ballet dancers $(N=29)$ using self-report measures of these constructs. They found that physical stress and specific personality traits were correlated with higher injury rates. For instance, dancers with a history of stress fractures were described as having personality characteristics such as dominant, enterprising, assertive, adjusted, and extraverted. The researchers also concluded that these personality traits or qualities, when taken to an extreme, could contribute to greater injury risk. To elaborate, dancers who are "overachievers" may be at a higher risk for injuries than dancers lower in traits like dominance and extraversion given the continual competition in training and performance; because training and performance are yearlong, dancers' drive to improve their physique, artistry, and technique is ongoing (Hamilton et al., 1989). Without proper rest, recovery, and general self-care, what once was hunger and determination can become unhealthy obsessive passion (Akehurst \& Oliver, 2014).

Building on these findings, Patterson, Smith, Everett, and Ptacek (1998) have suggested that ballet dancers are a logical population in which to study psychosocial factors as predictors of injury due to the stressful nature of the dance environment (e.g., Hamilton et al., 1989). Patterson and colleagues (1998) conducted a study in which they hypothesized that the role of social 
support for dancers would be important in a "highly competitive and demanding profession, where the greatest rewards are often peer acceptance and accolades from dance masters and critics (Hamilton et al., 1989)" (p. 102). The researchers used a prospective study design to investigate the relationships between life events, social support, and injury in a sample of dancers from a southwestern US ballet company $(N=46)$. They found that recent negative life events were strong predictors of injury. Stressful life events explained approximately $50 \%$ of the variance in injuries that dancers with low levels of social support experienced over an eightmonth period.

Patterson and colleagues' (1998) study supports the idea that psychosocial factors can be meaningful predictors of injury risk among dancers. The researchers also implied that high levels of social support, in some cases, may safeguard dancers against injury. These findings imply that social, environmental, or psychological adjustments within dance schools and companies can enhance dancer well-being, and in turn, potentially impact dance injury occurrence.

In summary, it is essential that the culture within the dance environment-including its social norms, technique, occupational considerations, and mentality - be considered when investigating injury among dancers, and more generally when conducting research with this population. Dancers are both athletes and artists (Dick et al., 2013) and therefore they are constantly balancing both physical and mental demands. There appears to exist a consensus among researchers that an understanding and appreciation for this subculture-including perfectionistic striving (Anderson \& Hanrahan, 2008), pain tolerance (Wainwright, Williams, \& Turner, 2005), and artistic demands (Hamilton et al., 1989) — is necessary to contextualize study findings and implications. 


\section{Pain and Pain Appraisal}

As is evident in the previous section on dance culture, pain is often viewed as a necessary, unavoidable part of a dancer's training and performance regimen. After all, dancers participate in the type of activity "which demands diametrically opposed physical traits... strong, yet flexible; muscular, yet skeletal; durable, yet delicate; and powerful, but gravity defying" (Katella, 2014, p. 27). Their movements must be precise, and therefore repetition of technical skills and positions makes up a large part of dance classes and rehearsals. Repetition can be a positive part of training, leading to the development of muscle memory and increased confidence in choreography. However, on the negative side, repetition may lead to wear-and-tear of bones and tendons in a dancer's body, and subsequently result in overuse injuries and/or chronic pain.

In their study of pain appraisal and coping in a sample of dancers $(N=51)$, Anderson and Hanrahan (2008) discuss the conceptual difference between performance pain and injury pain, stating that performance pain is an accepted norm in the greater sport culture, with "the aches and pains related to athletic performance" often being appraised as an "integral part of the life of an athlete" (p. 9). What makes the experience of pain and any subsequent appraisal(s) of pain even more complicated is the fact that the dancer is the sole person who can decide the level of discomfort or distress and whether or not to report it or treat it.

Using the Pain Appraisal Inventory (PAI; Unruh \& Ritchie, 1998), the Pain Control Subscale from the Survey of Pain Attitudes (Jensen, Karoly, \& Huger, 1987), and the Sports Inventory for Pain (Meyers, Bourgeois, \& LeUnes, 2001), Anderson and Hanrahan (2008) sought to gather self-reported information from their sample of dancers in order to explore the possible relationships between the types of pain that dancers experienced, their appraisals of pain, and the coping styles used to deal with the pain. An important finding from their study was 
that participants did not appear to distinguish between regular performance pain and injury pain. "Consequently, the cognitive appraisal made of the pain, or the use of pain coping styles, did not differ according to the pain characteristics" (p. 14). This result is critical to the understanding of pain and pain appraisal among dancers, as it illustrates the limits of a dancer's medical judgment about their perceived pain (i.e., is it a symptom of an injury that needs treatment?) and the extent to which overconformity to the sport ethic of "no pain, no gain" may influence a dancer's decision to keep rehearsing or performing and deal with the consequences later on.

Findings from a qualitative study by Wainwright, Williams, and Turner (2005) reinforce the possible influence of the sport ethic on dancers' appraisals, and reflect the impact that dance culture has on the connection between perceived pain and performance:

Some dancers are just so used to working with the failing bodies that 'keeping going' becomes its own reward. Paradoxically, dancers also sometimes felt in peak physical condition and to be dancing at their very best just before their bodies gave out under the stress. (p. 53)

This quote expresses the interplay of the dancer's mind and body with the larger motivational climate present in the dance studio or stage. These factors figure prominently in the recognition and subsequent appraisal of pain, and highlight the balance between individual judgment and collective norms. These same factors are also reflected in multiple models of injury, given that injury is a physical and subjective experience for many athletic performers, including dancers.

\section{Models of Injury}

Researchers and sport psychology practitioners have developed several models to better understand and conceptualize injury among athletes and performers (e.g., Andersen \& Williams, 1988; Mainwaring, Krasnow, \& Kerr, 2001). Typically, these models are classified into two 
main categories: response and prevention. Response models aim to determine how an athlete might respond to injury and subsequent rehabilitation, while prevention models aim to explain what factors lead to injury and thus how athletes can work to prevent injuries from happening. Some models include both a response and prevention focus (e.g., Wiese-Bjornstal et al., 1998), capturing both antecedent factors and reactions to the injury experience. Given the focus of the present study, this section will begin with an example of a response model, then move to a blended model, and conclude with a prevention model that serves as this study's theoretical framework.

Mainwaring, Krasnow, and Kerr (2001) developed a model of injury specific to dancers, focusing on the factors that can predict or describe dancers' responses to injury. The model addresses personal and situational factors that can increase or decrease the intensity of the injury response. Personal factors within this model include personality, coping skills, identity, nutrition, and stressors. Situational factors include social support, dance culture and milieu, information about the injury, and the injury treatment. The researchers' comprehensive model also addresses the impact of the injury itself on the dancer's cognitive appraisal. For instance, it may be important to consider the dancer's history of injury, the type of injury currently sustained, the location in which that injury occurred, and the severity of the injury (i.e., minor versus major). These injury factors, combined with the personal and situational factors described, are likely to influence a dancer's cognitive appraisal of the injury experience and his or her emotional response (e.g., anger, optimism, shock, fear). Ultimately, these factors may influence a dancer's willingness to engage in and adhere to injury rehabilitation, which research has shown can impact the process of returning to sport (e.g., Podlog, Heil, \& Schulte, 2014), or in this case, returning to dance. 
Similar to Mainwaring and colleagues' (2001) model, Wiese-Bjornstal, Smith, Shaffer, and Morrey (1998) developed an integrated model of sport injury that reflects many personal and situational factors that impact the response to injury and subsequent recovery outcomes. This model also identifies four antecedent factors (personality, history of stressors, coping resources, and interventions) that can affect the stress response and injury susceptibility and the recovery process via cognitive appraisal. At the core of this model is the athlete's cognitive appraisal of the injury. The researchers suggest that personal and situational factors such as the athlete's injury history, athletic identity, physical health status, sport type, coach influences, and scholarship status interact with the antecedent factors to impact cognitive appraisal, and in turn predict the athlete's emotional and behavioral response. This model is undoubtedly complex and multidimensional, reflecting the uniqueness of each athlete's injury response and rehabilitation despite the regularity of injuries across sports.

Wiese-Bjornstal and colleagues (1998) credit Andersen and Williams (1988; Williams \& Andersen, 1998) with illuminating the impact of pre-injury factors on the injury experience. Indeed, Andersen and Williams (1988) developed and then revised (1998) a model of stress and injury that has become a benchmark among sport and exercise psychology professionals (e.g., Smith \& Bar-Eli, 2007). The model explores multiple antecedents of injury, namely personality (e.g., anxiety, hardiness, perfectionism); coping skills (i.e., coping strategies and resources); and history of stressors (i.e., life events such as death of a family member or moving to a new place). Williams and Andersen (1998) proposed that when a potentially stressful athletic situation (i.e., injury susceptibility) is present, one's cognitive appraisal and physiological/attentional changes (e.g., narrowed focus, increased muscle tension), in conjunction with these antecedent factors, affects the likelihood of injury. Furthermore, the model illustrates the use of interventions (e.g., 
psychological skills training programs) to address the antecedent factors and the stress response itself, consequently influencing the likelihood of injury susceptibility and injury outcomes. This model provides the context from which the present study is conducted.

The model's framework illustrates the potential for short-term psychological skills intervention programs to have a positive impact on injury frequency. Previous research by Noh, Morris, and Andersen (2007) illustrates the applicability of this model to real-world findings. The researchers conducted an intervention-based study in which they randomly assigned dancers with low levels of coping skills $(n=30)$ to one of two conditions, either an autogenic training only condition or a broad-based coping skills condition, the latter of which consisted of autogenic training, imagery, and positive self-talk. Dancers from a nearby dance school $(n=15)$ were utilized as a control group.

Following the delivery of these interventions three times per week across a 12-week period, the researchers found that the broad-based coping skills group reported enhanced coping skills, specifically in the areas of peaking under pressure, having confidence, achievement motivation, concentrating, and coping with adversity. Additionally, they found that the dancers in the broad-based coping skills group spent less time being injured than those dancers who were assigned to the control group (Noh, Morris, \& Andersen, 2007). These results reflect the veracity of Williams and Andersen's (1998) model and establish a new line of research focused on examining the applicability of such a model to a population other than collegiate or elite sport athletes, namely dancers.

In conclusion, various models from the sport psychology literature have demonstrated that personal, psychosocial, and environmental factors can affect injury risk and recovery. In particular, the aforementioned researchers have illustrated the role that coping skills can play in 
the cognitive appraisal process, which is an area where interventions may be effective in reducing injury outcomes. The present study utilized the Williams and Andersen (1998) model due to its status in the literature, and moreover its emphasis on the link between stress and injury, since the study sought to address dancers' stress response and provide them with psychological skills to manage a variety of physical and psychosocial demands. The next section will discuss short-term interventions, specifically focusing on their development, delivery, and effectiveness. This overview will provide evidence and context for the present study's purpose and methodology.

\section{Short-Term Interventions}

Short-term interventions are one method for packaging and delivering new and/or useful information to individuals, and have the potential to create behavior change (Nemet et al., 2005; Pinkerton \& Rockwell, 1994). In addition to studies in sport psychology, there is evidence to support the use and efficacy of short-term interventions across other fields of study, including but not limited to medicine (e.g., Crowther, 1983), nutrition (e.g., Oenema, Tan, \& Brug, 2005; Torres-McGehee et al., 2011), and athletic training (Johnson, 2000). This section will explore select studies from across these research domains, and conclude with a discussion of the definition of "short term" as it applies to intervention programs targeting behavior change.

In the field of medicine, many short-term behavioral interventions have been used to modify patient behavior over time. For instance, Crowther (1983) examined changes in systolic and diastolic blood pressure readings in a sample of persons diagnosed with essential hypertension $(N=30)$ over an eight-week time frame. Each participant was assigned to one of three intervention groups, namely stress management training and relaxation imagery, relaxation imagery only, and weekly blood pressure checks (which was considered the control group). 
Crowther found that participants assigned to both of the treatment groups had significantly reduced systolic and diastolic blood pressure readings during treatment and were better at maintaining diastolic blood pressure reductions at follow up compared to participants assigned to the control condition.

Studies in nutrition have used shorter time frames, in some cases delivering interventions lasting three to four weeks total. For example, Torres-McGehee and colleagues (2011) conducted a four-week intervention (consisting of eight 45-minute sessions) with female collegiate auxiliary dancers (e.g., majorettes, dance teams, color guard) $(N=40)$ who were assigned to either a control group $(n=19)$ or a treatment group $(n=21)$. Both groups completed surveys aimed to measure eating disorders, depression, and nutritional and disordered eating knowledge. The treatment group received a team-centered program on sport nutrition, exercise, and disordered eating consequences. At the end of the four weeks, both groups completed the measures again, and the researchers found that the dancers in the treatment group reported lower means scores on several eating-disorder related characteristics and behaviors, namely depression, body dissatisfaction, maturity fears, and drive for thinness; they also scored higher on nutrition and eating disorder knowledge as measured by a nutritional knowledge survey (Shifflett, Timm, \& Kahanov, 2002) and the Eating Disorder Knowledge Questionnaire (Turk, Prentice, Chappell, \& Shields, 1999) (Torres-McGehee, Green, Leaver-Dunn, Lepper, Bishop, \& Richardson, 2011). Also targeting changes in nutritional patterns and thinking, Oenema, Tan, and Brug (2005) conducted a web-based computer-tailored intervention focused on nutrition-related behaviors of a sample of Dutch adults $(N=782)$ over a three-week period. Participants were assigned to one of three groups (intervention, information control, no information control). The researchers took baseline measurements, distributed a tailored CD-ROM to those in the 
intervention group, and collected post-intervention data after the three-week period. The researchers reported that the participants assigned to the intervention group reported statistically significant increases fruit and vegetable intake.

Finally, brief interventions, lasting approximately three sessions, are evident in the athletic training and injury rehabilitation literature. Johnson (2000) conducted a study in which competitive athletes with long-term injuries $(N=58)$ were assigned to either an experimental $(n$ $=14)$ or control $(n=44)$ group, with the experimental group receiving education on the following skills: stress management and cognitive control, goal setting, and relaxation/guided imagery. Participants in the experimental group met with the same sport psychologist individually over the course of three sessions (total time frame was not reported), focusing on one skill per session for approximately 15 to 25 minutes. Statistical analyses revealed that the experimental group reported overall higher mood compared to the control group at both the midpoint and the conclusion of the intervention program. The researcher found a statistically significant difference between the experimental and control groups for relaxation/guided imagery. While Johnson fails to report a total time frame for the study, the researcher states: "research on brief psychotherapy indicates that models in which patients receive 3 contacts have the potential to create changes" (p. 216).

The studies described in this section, in conjunction with multiple intervention studies published in the sport psychology literature (e.g., Johnson, Ekegren, \& Andersen, 2005; Maddison \& Prapavessis, 2005), provide evidence of observable changes in thought and behavior within a relatively short period of time. According to Johnson, Ekegren, and Andersen (2005), "brief therapy models seem well suited for sport interventions and have been used, in various forms, in the past" (p. 32). Thus it was deemed appropriate for the present study to adopt 
a very brief psychological intervention model. However, it should be noted that there is a lack of research focused on interventions specifically designed for dancers, and therefore the present study utilized this timeframe based on the assumed best practices available in the literature.

One factor that remains somewhat unclear across these studies is time frame; it appears that different protocols and methods result in different timelines. Researchers have concluded that an average of five to 15 sessions is a typical, recommended range for brief therapy (Pinkerton \& Rockwell, 1994). Furthermore, within a college setting, more than half of students who seek mental health services are seen for five or fewer sessions (Pinkerton \& Rockwell, 1994). Following these guidelines, it is necessary to evaluate the various intervention programs that currently exist for the purpose of behavior change. In the subsequent sections, respective overviews of a) psychological skills training programs, b) stress inoculation protocols, and c) cognitive behavioral therapy programs or interventions will be offered. The purpose of these sections is to discuss information about the content and structure of these types of interventions, and explore some of the important findings that have resulted from them.

\section{Psychological Skills Training}

It is important to examine the use of psychological skills training programs in a variety of performance settings so as to appropriately determine the development of new interventions for performers (such as dancers). Currently, there is a plethora of research that explores the effectiveness of mental skills programs with athletes competing at different levels across sport types (e.g., Kar \& Bhukar, 2013). In their review of 19 published studies utilizing interventions with competitive athletes, Greenspan and Feltz (1989) defined interventions as "actions initiated by someone other than the athlete that focused on psychological skills in an attempt to improve the athlete's performance during competition" (p. 219). This section will contain and overview 
of several intervention studies that employ various mental skills aimed at reducing the risk of injury, given the focus of the present study.

Currently, literature on mental skills is populated with several topics or skills, including but not limited to: self-talk (e.g., Rogerson \& Hrycaiko, 2002; Theodorakis, Chroni, Laparidis, Bebetsos, \& Douma, 2001; Johnson, Hrycaiko, Johnson, \& Halas, 2004; Landin \& Herbert, 1999; Hamilton, Scott, \& MacDougall, 2007; Hatzigeorgiadis, Zourbanos, Galanis, \& Theodorakis, 2011), imagery (Vealey \& Greenleaf, 2001; Weinberg \& Gould, 2011), autogenic training (e.g., Goldbeck \& Schmid, 2003; Kanji, White, \& Ernst, 2006; Krampen, 1996), progressive muscle relaxation (e.g., Carlson \& Hoyle, 1993; Emery, France, Harris, Norman, \& VanArsdalen, 2008; Wilk \& Turkoski, 2001), goal setting (e.g., Evans \& Hardy, 2002), and positive social support (e.g., Hogan, Linden, \& Najarian, 2002). The following pages will describe a few select studies in which these types of skills were used in intervention programs with a focus on injury prevention and/or reduction.

Some researchers have targeted "at risk" athletes for the delivery of intervention programs, specifically examining the impact of stress-management skills on injury outcomes (Johnson, Ekegren, \& Andersen, 2005). For instance, Johnson, Ekegren, and Andersen (2005) examined the effectiveness of an injury prevention program during a 19-week competitive season with a sample of soccer players $(N=32)$ who were considered "at risk" given their psychosocial profiles, as measured by The Sport Anxiety Scale (SAS; Smith, Smoll, \& Schutz, 1990), the Athletic Coping Skills Inventory (ACSI-28; Smith, Schutz, Smoll and Ptacek, 1995), and the Life Events Survey for College Athletes (LESCA; Petrie, 1992). The researchers introduced the athletes to mental skills —including somatic and cognitive relaxation, stress management, goal setting, attribution and self-confidence training — across six to eight 
individualized sessions lasting 45 to 90 minutes each. The researchers reported that the players who received the intervention program had fewer injuries over the course of the season when compared to those athletes who were randomly assigned to the control group. This finding lays groundwork for the implementation of individualized, in addition to group-focused, interventions with competitive athletes.

Maddison and Prapavessis (2005) conducted a month-long program with a sample of rugby players $(N=48)$ in order to examine the effectiveness of cognitive-behavioral stress management intervention. During each of the six sessions, which lasted 90 to 120 minutes each, the players were introduced to skills including somatic and cognitive based relaxation, imagery, progressive muscle relaxation, autogenic training, and goal setting. Following the intervention program, the researchers reported an observed condition effect, with the players in the treatment group missing less playing time than the players in the control group. However, this condition effect was limited to time missed, as this result was not upheld for number of reported injuries.

Other intervention studies with a focus on injury prevention that capitalize on stressmanagement protocols, including those that promote mindfulness as a psychological skill. Ivarsson, Johnson, Andersen, Fallby, and Altemyr (2015) conducted a mindfulness-based program focused on injury reduction in a sample of junior elite soccer players $(N=41)$ across a series of seven sessions. Players in the treatment group received training on mindfulness, acceptance, and commitment, while the players in the control group received training on attentional control as it applied to their sport. At the conclusion of the program, the researchers found that over two-thirds of the players in the treatment group remained injury free; in contrast, only $40 \%$ of participants in the control group did not experience an injury (Ivarsson et al., 2015).

In a review article of mindfulness and acceptance models in sport psychology, Gardner 
and Moore (2012) describe multiple studies that utilized a mindfulness approach to performance enhancement in contrast to the more "traditional" cognitive behavioral or stress management frameworks. While none of the studies reviewed explore findings specifically related to injury reduction, the findings include reports of increased concentration levels, decreased worry, and increased mindful awareness. These findings can be tied back to Williams and Andersen's (1988) model of stress and athletic injury under the hypothesis that adopting a mindfulness attitude in sport may influence antecedent factors (e.g., coping skills) of injury, and in some cases, change athletes' cognitive appraisal of stressful situations, resulting in reduced injury risk.

Given the aforementioned findings, it is evident that interventions that utilize psychological skills training can be useful for the reduction of injury among athletes in a variety of sports and settings. Whether the reported results were statistically significant or clinically meaningful, each study contributed to the existing literature on the efficacy of using mental skills as a way to moderate the relationship between psychological stress and physical injury. Much can be learned from these studies, including their selected methodologies, presentation of results, and overall procedure. In order to examine the feasibility of using psychological skills training in the present study, it is necessary to explore some of the studies in which these types of interventions were delivered to dance populations.

\section{Psychological Skills Training Interventions in Dance}

At present, there is a lack of literature exploring the effectiveness of short-term psychological skills interventions in non-sport populations (see Noh, Morris, \& Andersen, 2007). Hays (2002) has cited only a few researchers who have examined psychological skills and psychosocial factors as they relate to injury in the performing arts. Additionally, Hays (2002) has referred to psychological skills training interventions with performing artists as frequently being 
"reparative" rather than "preventive" in nature (p. 301). There exists a need for more research on injury as it relates to self-monitoring, relaxation training, and performance anxiety reduction. Similarly, Noh, Morris, and Andersen (2003) expressed the need to "develop and test intervention programs that can reduce dance injuries," stating that "the development of social support and more effective coping strategies (e.g., imagery, self-talk) may have benefits that reduce dancers' high levels of stress and the frequency of injury” (p. 39).

Noh and colleagues (2005) answered their own call to action by conducting a study with a sample of ballet dancers $(N=105)$ from a variety of training backgrounds (i.e., professional, university, ballet institute). The researchers measured stress, anxiety, and social support, and explored whether these factors could predict injury frequency and duration over a 10-month period. They found that "freedom from worry" and "confidence" predicted injury frequency, and "freedom from worry" and "negative dance stress" predicted injury duration within their sample (Noh, Morris, \& Andersen, 2005). The researchers' findings relate back to two models of injury previously discussed (Andersen \& Williams, 1988; Wiese-Bjornstal et al., 1998), and emphasize the areas in which sport psychology consultants and other helping professionals can be valuable to dancers.

As previously discussed, Noh and colleagues continued this line of research by investigating the effects of two psychological skills interventions delivered to a group of ballet dancers $(N=35)$ with a focus on injury reduction. Dancers were assigned to one of three conditions, namely control $(n=12)$, autogenic training $(n=12)$, and broad-based coping skills $(n$ $=11$ ). The broad-based coping skills condition consisted of autogenic training, imagery, and selftalk over the course of the intervention. The intervention lasted for 12 weeks, after which time participants were asked to practice the skills they had learned at least three times per week for an 
additional 12 weeks. Injuries for each dancer were recorded throughout the study.

The researchers measured multiple factors related to Williams and Andersen's (1998) stress-injury model. These measures included the Athletic Coping Skills Inventory-28 (ACSI-28; Smith, Schutz, Smoll, \& Ptacek, 1995), an injury survey asking dancers to detail their injury experiences in the previous 12 months, an injury report form used to record injury frequency and duration, and an adherence diary for dancers to log practice of the skills learned (and in the control condition, to log their dancing for practice and performance). Dancers in the autogenic training and broad-based coping skills groups participated in the intervention three times per week for 12 weeks, with the average session time being 25 minutes for autogenic training and 40 minutes for broad-based coping skills.

The researchers found the broad-based coping skills condition to be most effective, crediting it with enhancing dancers' coping skills (Noh, Morris, \& Andersen, 2007). They reported that an examination of gain scores on ACSI-28 subscales showed that the broad-based coping skills intervention increased dancers' coping skills in the areas of (1) peaking under pressure, (2) coping with adversity, (3) confidence and achievement motivation, and (4) concentration more so than the autogenic training intervention and controls group. Additionally, they found that the dancers assigned to the broad-based coping skills group spent less time being injured compared to dancers who were assigned to the control group (Noh, Morris, \& Andersen, 2007). "The focus on enhancing coping skills that are known to influence practice and performance appeared to help dancers cope more effectively with the demands of the dance environment and reduce injury incidence" (Noh, Morris, \& Andersen, 2007, p. 28).

Despite serving as inspiration for the present study, Noh, Morris, and Andersen's (2007) results are limited to data collected from Korean dancers. The present study therefore sought to 
fill a gap in the literature, where diverse samples of dancers are currently lacking. The present study focused on dancers who were training and performing in the Mid-Atlantic region of the United States. As such, any profoundly different results could inform practitioners and scholars.

Following from the information presented on psychological skills training programs in this section, it is necessary to consider other programs and protocols that might also suit a dance population seeking to reduce stress and injury susceptibility. The next section will therefore describe stress inoculation training programs as one alternative to a psychological skills training model of intervention. The section will not only offer a definition of stress inoculation protocols, but also provide a review of select studies that have utilized this approach.

\section{Stress Inoculation Training}

Among the various brief therapy models described in the literature, researchers have identified stress inoculation training as one way to deliver information and observe change quickly and effectively. Stress inoculation training programs consist of three main phases: (1) conceptualization/education, (2) rehearsal, and (3) application (Meichenbaum, 1977; 1985). In the first phase, students gain an understanding of stress in a psychoeducational manner (Mace \& Carroll, 1986). In the second stage, students are introduced to a variety of coping skills deemed appropriate to their particular problem(s) (Mace \& Carroll, 1986). For instance, if preperformance anxiety is a concern, relaxation techniques such as diaphragmatic breathing or imagery may be taught. In the third and final phase, students are encouraged to practice the coping skills that they have learned in somewhat stressful environs (Mace \& Carroll, 1986).

In this way, stress inoculation training promotes the development of coping resources and responses to address and/or reduce current problems, and to buffer the effects of stressful situations on the student in the future (Meichenbaum, 1985). Training programs generally range 
in length from eight to 15 sessions, with each session averaging 75 to 90 minutes in length (Meichenbaum, 1993). It should be noted that, while stress inoculation training does have a specific structure (via three phases), it also provides flexibility in content and delivery. According to Mace and Carroll (1986):

The procedure can be modified and applied to a wide variety of stress-related problems... It is this flexibility which makes stress inoculation training potentially very valuable for us in sport where competitors often suffer a loss of performance through stress. (p. 115)

As an illustration of this flexibility, Mace and Carroll (1986) delivered eight sessions of stress inoculation training to two squash players, who both reported suffering from anxiety when competing. Before implementing the program, the researchers measured anxiety with the StateTrait Anxiety Inventory (Spielberger, Gorsuch, \& Lushene, 1970) prior to five different competitions; following the intervention, the athletes once again completed the measure before five different competitions. The researchers reported that the athletes experienced less anxiety and increased confidence in coping with nerves or anxiety after the intervention. Additionally, the two athletes had been matched to two control subjects, and these control subjects did not show similar reductions in anxiety (Mace \& Carroll, 1986).

In a similar study, Mace, Eastman, and Carroll (1986) reported success in a case study with a 12-year-old, female gymnast. The researchers used a stress inoculation training program to help the athlete "regain her form" following several injuries and developing negative images and self-statements regarding her performance (p. 139). Over eight sessions, the researchers guided the athlete through the three phases, and observed subsequent changes in the athlete's mentality and physical performance. Mentally, the researchers reported a shift in the athlete's 
comments from negative to more positive. Physically, they report that she regained a specific skill on the uneven bars that caused difficulty during the application phase of training (Mace, Eastman, \& Carroll, 1986).

Moving away from a single-subject design, Kerr and Leith (1993) examined the effects of a stress inoculation training program on athletic performance, mental rehearsal, competitive anxiety, and attentional focus in a sample of competitive gymnasts $(N=24)$. Gymnasts were matched for age, gender, and ranking, and then assigned to either an experimental or control group. Gymnasts in the experimental group met individually with the experimenter two times per month for eight months, totaling 16 sessions per athlete. The researchers collected data on gymnasts' performance (via rankings/scores from three distinct competitions), mental rehearsal skills (The Sport Imagery Questionnaire; Martens, 1982), competitive anxiety (Sport Competition Anxiety Test; Martens, 1977), and attentional focus (Test Anxiety Scale; Sarason, 1958).

Following data analysis, the researchers found that gymnasts in both groups improved in performance over time, but the gymnasts in the experimental group had significantly greater improvement compared to gymnasts in the control group. Furthermore, gymnasts in the experimental group indicated better mental imagery skills than gymnasts in the control group at time 3 (post-intervention), as well as "significantly better task-relevant foci” (p. 228). The researchers concluded that their intervention, based on Meichenbaum's (1985) guidelines, could be successful at improving athletic performance, although it remains unclear which parts of the intervention were most influential (Kerr \& Leith, 1993).

Building on the previous study, Kerr and Goss (1996) examined the effects of a stress inoculation program on stress levels and injuries in a sample of male and female competitive 
gymnasts $(N=24)$ ranging from 16 to 25 years of age. The researchers collected information on gymnasts' injuries (each occurrence and total number of days missed), life stress (The Life Experiences Survey; Sarason, Johnson, \& Siegel, 1978), and athletic stress (Athletic Experiences Survey; a 49-item measure developed for the study). Gymnasts were assigned to either the experimental or control group, using a matched-pairs design for gender, age, and ranking. Over the course of eight months, athletes in the experimental group met individually with a female experimenter on a bi-weekly basis to participate in one-hour sessions. Sessions included skills such as thought control, imagery, and cognitive restructuring (Kerr \& Goss, 1996).

Interestingly, $100 \%$ of gymnasts reported sustaining at least one injury during the eightmonth period. While there were no significant differences between groups on injury, gymnasts in the experimental group reported "significantly less negative athletic stress, less total negative stress and a trend to more positive athletic stress" at the final assessment, which took place at the end of the season upon National Championships (p. 114). The experimenters cited multiple limitations and challenges within their study, but remained optimistic that studies that expand and improve upon their methodology and sampling procedures will incite more intervention programs for injury prevention and/or reduction (Kerr \& Goss, 1996).

The purpose of this section was to describe and examine the applicability of stress inoculation training programs across various athletic populations, with particular consideration given to the recommended timeframe and relevant skills taught. It is clear from the described studies that interventions that follow a stress inoculation protocol, and more specifically the guidelines enumerated by Meichenbaum $(1977 ; 1985)$, can lead to important findings and encourage improvements for future research with performers of all kinds, including dancers. 
Indeed, it is evident that stress inoculation can serve as the foundation of many important studies related to cognitive appraisal and/or behavior change. The next section will provide an overview of a different type of method or approach: cognitive-behavioral.

\section{Cognitive Behavioral Approaches}

This section is intended to provide an overview of cognitive behavioral therapy as a theoretical approach or framework through which to view to performance enhancement in athletics, and group-based intervention programming. The use of cognitive behavioral therapy (CBT) programs transcends any particular field of study, and has applicability across domains including — but not limited to — counseling, rehabilitation, and sport and exercise psychology.

In terms of CBT's applicability to athletics, Brown (2011) has stated: "Performance is mental" and that "cognitive-behavioral strategies are fundamental to sport" (p. 113). Brown (2011) further states that many performers are already using psychological skills when they first meet a sport psychologist or performance consultant. Therefore, it becomes a goal of the collaboration to examine the ways in which these skills are presently being used, and help the performer to refine or adjust these skills to benefit or enhance their mentality.

Greenspan and Feltz (1989) have also provided their thoughts on the importance of cognitive-behavioral approaches in sport. The researchers claim that sport psychology professionals have often undertaken the task of delivering mental skills programs without examining the literature on best practices when working with a specific population. In their review of 19 published studies, Greenspan and Feltz (1989) concluded that cognitive restructuring (e.g., self-talk) programs and relaxation-based interventions may be effective with competitive athletes.

Neil, Hanton, and Mellalieu (2013) adopted a cognitive-behavioral framework for an 
investigation of cognitive/emotional appraisal and athletic performance with a sample of male golfers $(N=4)$ who were competing in a UK national championship. The researchers described their decision to select golfers for the intervention program given that golf "provides numerous and ever-changing stressors with which performers need to cope" (p. 109). Interestingly, this description somewhat parallels the experience of individual dancers, who may be asked to adjust to new costumes and footwear, changes in choreography or partnerships, and the like. To meet these demands, Neil and colleagues (2013) suggest that performers — and in their case specifically, golfers - may benefit from identifying and then modifying their self-talk in order to experience behavioral consequences that more appropriately fits the demands of competition: Indeed, an assumption of cognitive restructuring is that reorganizing and restructuring verbal statements about oneself and one's world will result in a corresponding reorganization of behavior with respect to one's world...the emphasis of this strategy is to focus on the negative thoughts of an individual in relation to the environment, and to alter these through a constructive narrative. (p. 108).

The researchers developed an intervention program with three distinct phases: (1) observation of the participants, (2) psychoeducation about select mental skills, and (3) application and practice of the mental skills in competition. They quantified golfers' performance through a number of related indexes, including their score on each hole. Golfers were provided a booklet in which to rate their performance after they completed each round of golf. At the conclusion of the study, which spanned 34 weeks, the researchers found that golfers self-reported positive changes in their emotions/affect, and indicated that their attention had moved away from internal, negative thoughts and instead was moving to task-focused thinking. 
Within the collegiate environment, Perna, Antoni, Baum, Gordon, and Schneiderman (2003) used a three-week time frame to deliver a program inspired by a stress inoculation training framework, which has been previously discussed in this chapter. Specifically, the researchers examined the effects of a cognitive behavioral stress management intervention on injury and illness with a sample of male $(n=14)$ and female $(n=20)$ competitive collegiate rowers $(N=34)$. Athletes were randomly assigned to an experimental or control condition. Both groups were asked to complete the Life-Event Scale for College Athletes (LESCA; Petrie, 1992), Brief Assessment of Mood (BAM; Dean, Whelan, \& Meyers, 1990), and to provide blood samples prior to morning practices. Additionally, all athletes gave the researchers permission to access their medical information, and the head athletic trainer and university medical center recorded all injury and illness days (including office visits).

A licensed psychologist and a clinical psychology intern delivered the stress management program to the experimental group across seven structured sessions. Sessions included, but were not limited to, the following skills: progressive muscle relaxation, diaphragmatic breathing, emotive imagery, cognitive restructuring, and relapse prevention. Throughout the study, participants were asked to log their daily practice of the skills learned and to complete homework assigned for time outside of sessions. In tandem, the control group received one two-hour stress management presentation in an effort to prevent experimenter expectancy effects.

At the conclusion of the study, the researchers found that the rowers in the experimental group experienced "significant reductions in the number of illness and injury days and had half the number of health service visits" when compared to their control group counterparts (p. 70). These findings have implications for the present study, as Perna and colleagues (2003) results suggest that, when paired with self-initiated practice outside of the program, interventions rooted 
in a cognitive-behavioral framework can address, and possibly impact, the intensity and effects of physical and psychological stress.

Based on the available literature, it is evident that this theoretical approach can be useful to those who are receiving content in a variety of healthcare, athletic, and performance settings. It was selected as the primary approach for the present study given its usage in previous psychological skills interventions and its applicability to the physical and psychosocial factors that are often at play in the dance environment.

\section{Concluding Thoughts}

According to Simpson (2006), 98\% of dance injuries "are treated non-operatively" (p. 3), suggesting that tactics like rest, cross-training, rehabilitation, and injury prevention programming can be essential to a dancer's training and performance cycle. Therefore, the establishment of a short-term intervention for the prevention of dance injuries is a valuable resource for schools and companies across the US and internationally. To date, few researchers have attempted to develop, implement, and evaluate the effectiveness of such programs. This may come as a surprise, given the understanding that psychosocial stress has "an adverse effect on the dancer physically and contributes to a higher injury rate. Dancers who undergo psychosocial stressrelated interventions have less physical injuries and increased recovery from injuries" (Dick et al., 2013, p. 400).

Despite some perceived similarities to other athletes, such as gymnasts (e.g., Kerr \& Goss, 1996), dancers are not well represented in research focused on psychological skills training for the prevention of injury. This population is likely to benefit from receiving more information about psychosocial factors related to injury susceptibility, and injury reduction techniques such as those introduced to athletes somewhat regularly by sport psychologists. As stated by Johnson 
and colleagues (2005): "in contrast to the rich body of empirical studies on the psychological factors in the occurrence of sport injuries, few controlled intervention studies have examined relationships among psychological risk variables, prevention treatments, and injury outcomes" (p. 32).

More intervention studies are likely to lead to a better understanding of what psychosocial skills are effective, useful, and meaningful to dancers across a variety of settings. From the findings of this study and additional studies, researchers and practitioners can create and implement more programs and educational resources that target dancers' stress levels and coping skills as precursors to injury risk. It is ideal to have dancers engage in psychological skills training prior to pursuing a professional dance career so that they have time to learn, practice, and apply these tools to both their training and performance opportunities. Early introduction may not only allow for immediate benefits (e.g., lower perceived stress) but also for long term advantages (e.g., awareness of injury risk, increased confidence in coping with negative life events). The development and subsequent testing of the effects of injury prevention programs can help researcher and practitioners alike to keep dancers healthy; educate athletic trainers and physical therapists; extend dancers' careers; maintain or grow the job market for sport psychology practitioners; and decrease medical, health, and surgery costs for all who dance. 


\section{References}

Aalten, A. (2005). In the presence of the body: Theorizing training, injuries and pain in ballet. Dance Research Journal, 37(2), 55-72.

Akehurst, S., \& Oliver, E. J. (2014). Obsessive passion: A dependency associated with injuryrelated risky behavior in dancers. Journal of Sports Sciences, 32(3), 259-267.

Allen, N., Nevill, A., Brooks, J., Koutedakis, Y., \& Wyon, M. (2012). Ballet injuries: Injury incidence and severity over 1 year. Journal of Orthopaedic \& Sports Physical Therapy, 42(9), 781-A1.

Andersen, M. B., \& Williams, J. M. (1988). A model of stress and athletic injury: Prediction and prevention. Journal of Sport \& Exercise Psychology, 10, 294-306.

Anderson, R., \& Hanrahan, S. J. (2008). Dancing in pain: Pain appraisal and coping in dancers. Journal of Dance Medicine \& Science, 12(1), 9-16.

Bell, R. J., Skinner, C. H., \& Fisher, L. A. (2009). Decreasing putting yips in accomplished golfers via solution-focused guided imagery: A single-subject research design. The Journal of Applied Sport Psychology, 21(1), 1-14.

Brewer, B. W., Van Raalte, J. L., \& Linder, D. E. (1993). Athletic identity: Hercules muscles or Achilles heel?. International Journal of Sport Psychology, 24(2), 237-254.

Bronner, S., Ojofeitimi, S., \& Rose, D. (2003). Injuries in a modern dance company: Effect of a comprehensive management on injury incidence and time loss. The American Journal of Sports Medicine, 31(3), 365-373.

Brown, J. L. (2011). Cognitive-behavioral strategies. In J. K. Luiselli, \& D. D. Reed (Eds.). Behavioral Sport Psychology: Evidence-Based Approaches to Performance Enhancement (pp. 113-114). New York: Springer Science. 
Butt, J., Weinberg, R.S., \& Culp, B. (2010). Exploring mental toughness in NCAA athletes. Journal of Intercollegiate Sports, 3, 316-332.

Carlson, C. R., \& Hoyle, R. H. (1993). Efficacy of abbreviated progressive muscle relaxation training: A quantitative review of behavioral medicine research. Journal of Consulting and Clinical Psychology, 61(6), 1059-1067.

Carver, C. S. (1997). You want to measure coping but your protocol's too long: Consider the Brief COPE. International Journal of Behavioral Medicine, 4, 92-100.

Carver, C. S., Scheier, M. F., \& Weintraub, J. K. (1989). Assessing coping strategies: A theoretically based approach. Journal of Personality and Social Psychology, 56(2), 267283.

Center for Disease Control. (2015). Injury prevention \& control. Retrieved from http://www.cdc.gov/injury/index.html

Children's Hospital Colorado Orthopedics Institute. (2014). Sports Medicine Center: Dance. Retrieved from https://www.childrenscolorado.org/doctors-anddepartments/departments/orthopedics/programs/sports-medicine-center/sports-injurieswe-treat/dance/

Christoffel, T., \& Gallagher, S. S. (2006). Injury prevention \& public health: Practical knowledge, skills and strategies ( $2^{\text {nd }}$ ed.). Sudbury, MA: Jones and Bartlett Publishing. Connaughton, D., Wadey, R., Hanton, S., \& Jones, G. (2008). The development and maintenance of mental toughness: Perceptions of elite performers. Journal of Sports Sciences, 26(1), 83-95.

Coppel, D. B. (1980). Relationship of perceived social support and self-efficacy to major and minor stresses. Unpublished doctoral dissertation, University of Washington, Seattle. 
Creswell, J. W. (2007). Qualitative inquiry \& research design. Thousand Oaks, CA: Sage Publications.

Crocker, P. R. E., Kowalski, K. C., \& Graham, T. R. (1998). Measurement of coping strategies in sport. Morgantown, EV: Fitness Information Technology.

Crocker, P. R. E., Kowalski, K. C., \& Graham, T. R. (1998). Measurement of coping strategies in sport. Morgantown, EV: Fitness Information Technology.

Crowther, J. H. (1983). Stress management training and relaxation imagery in the treatment of essential hypertension. Journal of Behavioral Medicine, 6(2), 169-187.

Dean, J. E., Whelan, J. P., \& Meyers, A. W. (1990, September). An incredibly quick way to assess mood states: The incredibly short POMS. Paper presented at the meeting of the Association for the Advancement of Applied Sport Psychology, San Antonio, TX.

Derogatis L. (1983). The psychosocial adjustment to illness scale. Baltimore, MD: Clinical Psychometric Research.

Department of Health and Human Services (2014, March 17). Common sports injuries: Incidence and average charges. Retrieved from https://aspe.hhs.gov/sites/default/files/pdf/76816/ib_SportsInjuries.pdf

Dick, R. W., Berning, J. R., Dawson, W., Ginsburg, R. D., Miller, C., \& Shybut, G. T. (2013). Athletes and the arts: The role of sports medicine in the performing arts. Current Sports Medicine Reports, 12(6), 397-403.

DiFiori, J. P., Benjamin, H. J., Brenner, J., Gregory, A., Jayanthi, N., Landry, G. L., \& Luke, A. (2014). Overuse injuries and burnout in youth sports: A position statement from the American Medical Society for Sports Medicine. Clinical Journal of Sport Medicine, 24(1), 3-20. 
Ekegren, C. L., Quested, R., \& Brodrick, A., (2013). Injuries in pre-professional dancers: Incidence, characteristics, and consequences. Journal of Science and Medicine in Sport, $17,271-275$.

Emery, C. F., France, C. R., Harris, J., Norman, G., \& VanArsdalen, C. (2008). Effects of progressive muscle relaxation training on nociceptive flexion reflex threshold in healthy young adults: A randomized trial. Pain, 138(2), 375-379.

Encarnacion, M. L. G., Meyers, M. C., Ryan, N. D., \& Pease, D. G. (2000). Pain coping styles of ballet performers. Journal of Sport Behavior, 23, 20-32.

Evans, L., \& Hardy, L. (2002). Injury rehabilitation: A goal-setting intervention study. Research Quarterly for Exercise and Sport, 73(3), 310-319.

Erickson, J. (2015, March 16). The black swan effect. Huffpost Healthy Living. Retrieved from http://www.huffingtonpost.com/julia-erickson-/the-black-swan-effect_b_6866896.html

Faul, F., Erdfelder, E., Lang, A. G., \& Buchner, A. (2007). G*Power 3: A flexible statistical power analysis program for the social, behavioral, and biomedical sciences. Behavior Research Methods, 39, 175-191.

Gardner, F. L., \& Moore, Z. E. (2012). Mindfulness and acceptance models in sport psychology: A decade of basic and applied scientific advancements. Canadian Psychology, 53(4), 309-318.

Gay, L. R., \& Airasian, P. W. (2003). Educational research: Competencies for analysis and Applications. Upper Saddle River, NJ: Merrill/Prentice Hall.

Goldbeck, L., \& Schmid, K. (2003). Effectiveness of autogenic relaxation training on children and adolescents with behavioral and emotional problems. Journal of the American Academy of Child and Adolescent Psychiatry, 42(9), 1046-1054. 
Goldberg, A. S., Moroz, L., Smith, A. \& Ganley, T. (2007). Injury surveillance in young athletes: A clinician's guide to sports injury literature. Sports Medicine, 37(3), 265-278.

Gravetter, F. J., \& Forzano, L. B. (2013). Research methods for the behavioral sciences. Belmont, CA: Wadsworth, Cengage Learning.

Greenspan, M. J., \& Feltz, D. L. (1989). Psychological interventions with athletes in competitive situations: A review. The Sport Psychologist, 3, 219-236.

Grimm, L. G., \& Yarnold, P. R. (1995). Reading and understanding multivariate statistics. Washington, DC: American Psychological Association.

Grove, J. R., Main, L. C., \& Sharp, L. (2013). Stressors, recovery processes, and manifestations of training distress in dance. Journal of Dance Medicine \& Science, 17, 70-78.

Hamilton, L. H., Hamilton, W. G., Meltzer, J. D., Marshall, P., \& Molnar, M. (1989). Personality, stress, and injuries in professional ballet dancers. The American Journal of Sports Medicine, 17(2), 263-267.

Hamilton, R. A., Scott, D., \& MacDougall, M. P. (2007). Assessing the effectiveness of self-talk interventions on endurance performance. Journal of Applied Sport Psychology, 19(2), 226-239.

Hatzigeorgiadis, A., Zourbanos, N., Galanis, E., \& Theodorakis, Y. (2011). The effects of selftalk on performance in sport: A meta-analysis. Perspectives on Psychological Science, $6(4), 348-356$.

Hays, K. F. (2002). The enhancement of performance excellence among performing artists. Journal of Applied Sport Psychology, 14(4), 299-312.

Healthline. (2013, May 10). The understated injury: Overexertion. Retrieved from http://www.healthline.com/health/understated-injury-overexertion 
Heil, J. (1993). Psychology of sport injury. Champaign, IL: Human Kinetics.

Hogan, B. E., Linden, W., \& Najarian, B. (2002). Social support interventions: Do they work? Clinical Psychology Review, 22(3), 383-442.

IBM Corp. Released 2012. IBM SPSS Statistics for Windows, Version 21.0. Armonk, NY: IBM Corp.

Ivarsson, A., Johnson, U., Andersen, M. B., Fallby, J., \& Altemyr, M. (2015). It pays to pay attention A mindfulness-based program for injury prevention with soccer players. Journal of Applied Sport Psychology, 27(3), 319-334.

Jensen, M. P., Karoly, P., \& Huger, R. (1987). The development and preliminary evaluation of an instrument to assess patients' attitudes towards pain. Journal of Psychosomatic Research, 31, 393-400.

Johnson, U. (2000). Short-term psychological intervention: A study of long-term-injured competitive athletes. Journal of Sport Rehabilitation, 9, 207-218.

Johnson, U., Ekegren, J., \& Andersen, M.B. (2005). Injury prevention in Sweden: Helping soccer players at risk. Journal of Sport and Exercise Psychology, 1, 32-38.

Johnson, J. M., Hrycaiko, D. W., Johnson, G. V., \& Halas, J. M. (2004). Self-talk and female youth soccer performance. The Sport Psychologist, 18, 44-59.

Jones, G. (2002). What is this thing called mental toughness?: An investigation of elite sport performers. Journal of Applied Sport Psychology, 14(3), 205-218.

Kanji, N., White, A., \& Ernst, E. (2006). Autogenic training to reduce anxiety in nursing students: Randomized controlled trial. Journal of Advanced Nursing, 53(6), 729-735.

Kar, R., \& Bhukar, J. P. (2013). Effect of mental training on track \& field performance and mental skills: A single subject design. Academic Sports Scholar, 2(1), 1-7. 
Katella, K. (2014, Autumn). Dancing through pain. Yale Medicine, pp. 28-29.

Kerr, G., \& Goss, J. (1996). The effects of a stress management program on injuries and stress levels. Journal of Applied Sport Psychology, 8, 109-117.

Kerr, G., \& Leith, L. (1993). Stress management and athletic performance. The Sport Psychologist, 7, 221-231.

Krampen, G. (1996). Evaluation of the effectiveness of autogenic training in gerontopsychology: Its role in developmental intervention and its effects on development-related cognitions and emotions as well as psychosomatic complaints in the elderly. European Psychologist, $1(4), 243-254$.

Krasnow, D. H. (2005, December). Dance Rebooted: Initializing the grid. Ausdance National.

Krasnow, D., \& Mainwaring, L. (1990). Dance experiences survey. Retrieved January 2016 through personal communication with the survey developers.

Krasnow, D., Kerr, G., \& Mainwaring, L. (1994). Psychology of dealing with the injured dancer. Medical Problems of Performing Artists, 9, 7-9.

Landin, D., \& Hebert, E. P. (1999). The influence of self-talk on the performance of skilled female tennis players. Journal of Applied Sport Psychology, 11, 263-282.

Lazarus, R. S., \& Folkman, S. (1984). Stress, appraisal, and coping. New York, NY: Springer.

Mace, R., \& Carroll, D. (1986). Stress inoculation training to control anxiety in sport: Two case studies in squash. British Journal of Sports Medicine, 20, 115-117.

Mace, R., Eastman, C., \& Carroll, D. (1986). Stress inoculation training: A case study in gymnastics. British Journal of Sports Medicine, 20(3), 139-141.

Maddison, R., \& Prapavessis, H. (2005). A psychological approach to the prediction and prevention of athletic injury. Journal of Sport \& Exercise Psychology, 27, 289-310. 
Mainwaring, L. M., Krasnow, D., \& Kerr, G. (2001). And the dance goes on: Psychological impact of injury. Journal of Dance Medicine \& Science, 5(4), 105-115.

Malisoux, L., Frisch, A., Urhausen, A., Seil, R., \& Theisen, D. (2013). Injury incidence in a sports school during a 3-year follow-up. Knee Surgery, Sports Traumatology, Arthroscopy: Official Journal of the ESSKA, 21(12), 2895-2900.

Martens, R. (1982). Imagery in sport. Paper presented at the Medical and Scientific Aspects of Elitism in Sport Conference, Brisbane, Australia.

Martens, R. (1977). Sport Competition Anxiety Test. Champaign, IL: Human Kinetics.

Martin, R. (2009). Pirouetting with pain: Attitudes surrounding female ballet dancers dancing with pain. International Symposium on Performance Science. Retrieved from http://www.performancescience.org/ISPS2009/Proceedings/Rows/053Martin.pdf

McCubbin, H., Olson, D., \& Larsen, A. (1982). Family crisis oriented personal evaluation scales. In H. McCubbin (Ed.). Family assessment for research and practice (pp.194-207). Madison, WI: University of Wisconsin.

Meichenbaum, D.H. (1977). Cognitive-behavior modification. New York, NY: Plenum Press.

Meichenbaum, D.H. (1985). Stress inoculation training. New York, NY: Pergamon Press.

Meichenbaum, D.H. (1993). Stress inoculation training: A twenty year update. In R.L. Woolfolk, \& P.M. Lehrer (Eds.), Principles and practice of stress management ( $2^{\text {nd }}$ ed.) (pp.373406). New York: Guilford.

Melzack, R. (1987). The McGill Pain Questionnaire: From description to measurement. Anesthesiology, 103, 199-202. 
Meyers, M. C., Bourgeois, A. E., \& LeUnes, A. (2001). Pain coping responses of collegiate athletes involved in high contact, high injury potential sport. International Journal of Sport Psychology, 32, 29-42.

Mummery, K., Schofield, G., \& Perry, C. (2004). Bouncing back: The role of coping style, social support and self-concept in resilience of sport performance. Athletic Insight, Retrieved from http://www.athleticinsight.com/Vol6Iss3/BouncingBack.htm.

National Institutes of Health. (2014, November). What are sports injuries? Fast facts: An easyto-read series of publications for the public. Retrieved from http://www.niams.nih.gov/ Health_Info/Sports_Injuries/sports_injuries_ff.asp

Neil, R., Hanton, S., \& Mellalieu, S. D. (2013). Seeing things in a different light: Assessing the effects of a cognitive-behavioral intervention upon the further appraisals and performance of golfers. Journal of Applied Sport Psychology, 25, 106-130.

Nemet, D., Barkan, S., Epstein, Y., Kowen, G., \& Eliakim, A. (2005). Short- and long-term beneficial effects of a combined dietary-behavioral-physical activity intervention for the treatment of childhood obesity. Pediatrics, 115(4), 443-449.

Newall, P. (2016, January 07). An Eagles fan, trash talk and a battle over ballet. The Philadelphia Inquirer. Retrieved from http://articles.philly.com/2016-0107/news/69567114_1_pennsylvania-ballet-dancers-jonathan-stiles

Noh, Y., \& Morris, T. (2004). Designing research-based interventions of the prevention of injury in dance. Medical Problems of Performing Artists, 19(2), 82-89.

Noh, Y., Morris, T., \& Andersen, M. B. (2003). Psychosocial stress and injury in dance. Journal of Physical Education, Recreation, \& Dance, 74(4), 36-40. 
Noh, Y., Morris, T., \& Andersen, M. B. (2005). Psychosocial factors and ballet injuries. International Journal of Sport and Exercise Psychology, 3, 7-25.

Noh, Y., Morris, T., \& Andersen, M. B. (2007). Psychological intervention programs for reduction if injury in ballet dancers. Research in Sports Medicine, 15, 13-32.

Noh, Y., Morris, T., \& Andersen, M. B. (2002). Sources of stress and coping strategies of Korean ballet dancers. Annual conference of the Association for the Advancement of Applied Sport Psychology, Tucson, AZ.

Nordin-Bates, S. M., Quested, E., Walker, I. J., \& Redding, E. (2012). Climate change in the dance studio: Findings from the UK centres for advanced training. Sport, Exercise, and Performance Psychology, 1(1), 3-16.

Oenema, A., Tan, F., \& Brug, J. (2005). Short-term efficacy of a web-based computer-tailored nutrition intervention: Main effects and mediators. Annals of Behavioral Medicine, 29(1), 54-63.

Omar-Fauzee, M., Daud, W., Abdullah, R. \& Rashid, S. (2009). The effectiveness of imagery and coping strategies in sport performance. European Journal of Social Sciences, 9(1), 97-108.

Patton, M. Q. (2002). Qualitative research \& evaluation methods $\left(3^{\text {rd }}\right.$ Ed.). Thousand Oaks, CA: Sage Publications.

Patterson, E. L., Smith, R. E., Everett, J. J., \& Ptacek, J. T. (1998). Psychosocial factors as predictors of ballet injuries: Interactive effects of life stress and social support. Journal of Sport Behavior, 21(1), 101-112. 
Perna, F. M., Antoni, M. H., Baum, A., Gordon, P., \& Schneiderman, N. (2003). Cognitive behavioral stress management effects on injury and illness among competitive athletes: A randomized clinical trial. Annals of Behavioral Medicine, 25(1), 66-73.

Petrie, T. A. (1992). Psychosocial antecedents of athletic injury: The effects of life stress and social support on female collegiate gymnasts. Behavioral Medicine, 18(3), 127-138.

Pinkerton, R. S., \& Rockwell, W. J. (1994). Very brief psychological interventions with university students. Journal of American College Health, 42(4), 156-162.

Podlog, L., Heil, J., \& Schulte, S. (2014). Psychosocial factors in sports injury rehabilitation and return to play. Physical Medicine and Rehabilitation Clinics of North America, 25(4), $915-930$.

Rip, B., Fortin, S., \& Vallerand, R. J. (2006). The relationship between passion and injury in dance students. Journal of Dance Medicine \& Science, 19(1-2), 14-20.

Rivera, D. C., Alexander, J. L., Nehrenz, G. M., \& Fields, B. J. (2012). Dancers' perceptions of injuries. Journal of Music and Dance, 2(1), 9-12.

Rogerson, L. J., \& Hrycaiko, D. W. (2002). Enhancing competitive performance of ice hockey goaltenders using centering and self-talk. Journal of Applied Sport Psychology, 14(1), $14-26$.

Rosenbaum, M. (1980). A schedule for assessing self-control behaviors: Preliminary findings. Behavior Therapy, 11(1), 109-121.

Ruel, E., Wagner, W. E., \& Gillespie, B. J. (2016). The practice of survey research: Theory and applications. Thousand Oaks, CA: Sage Publications.

Russell, J. A. (2013). Preventing dance injuries: Current perspectives. Open Access Journal of Sports Medicine, 4, 199-210. 
Sarason, I. G. (1958). The Test Anxiety Scale: Concept and research. In C. D. Spielberger \& I. G. Sarason (Eds.), Stress and Anxiety (pp. 193-216). Washington, D.C.: Hemisphere Publishing Corp.

Sarason, I. G., Johnson, J. H., \& Siegel, J. M. (1978). Assessing the impact of life changes: Development of the Life Experiences Survey. Journal of Consulting and Clinical Psychology, 46(5), 932-946.

Schwarz, J. (2000, October 11). Ballet dancer injuries as common, severe as athletic injuries. $U W$ Today. Retrieved from http://www.washington.edu/news/2000/10/11/ballet-dancerinjuries-as-common-severe-as-athletic-injuries/

Shah, S. (2008). Caring for the dancer: Special considerations for the performer and troupe. Current Sports Medicine Reports, 7(3), 128-132.

Shah, S., Weiss, D. S., \& Burchette, R. J. (2012). Injuries in professional modern dancers: Incidence, risk factors, and management. Journal of Dance Medicine \& Science, 16(1), $17-25$.

Shifflett, B., Timm, C., \& Kahanov, L. (2002). Understanding of athletes' nutritional needs among athletes, coaches, and athletic trainers. Research Quarterly for Exercise and Sport, 73(3), 357-362.

Simpson, S. (2006). Dance injury management. Retrieved from http://cdn-flightdec.userfirst.co.nz/uploads/sites/danz/files/PDFs/InjuryMgmt.pdf

Smith, D., \& Bar-Eli, M. (2007). Essential readings in sport and exercise psychology. Champaign, IL: Human Kinetics. 
Smith, R. E., Schutz, R. W., Smoll, F. L., \& Ptacek, J. T. (1995). Development and validation of a multidimensional measure of sport-specific psychological skills: The Athletic Coping Skills Inventory-28. Journal of Sport \& Exercise Psychology, 17(4), 379-398.

Smith, R.E., Smoll, F.L., \& Schutz, R.W. (1990). Measurement and correlates of sport-specific cognitive and somatic trait anxiety: The Sport Anxiety Scale. Anxiety Research, 2, $263-$ 280.

Solomon, R. L., \& Micheli, L. J. (1986). Technique as a consideration in modern dance injuries. Physician and Sports Medicine, 14(8), 83-90.

Spielberger, C. D., Gorsuch, R.L., \& Lushene. R.E. (1970). Manual for the State-Trait Anxiety Inventory. Palo Alto, CA: Consulting Psychologists Press.

Tait, R. C., Chibnall, J. T., \& Krause, S. (1990). The Pain Disability Index: Psychometric Properties. Pain, 40(2), 171-182.

Theodorakis, Y., Chroni, S., Laparidis, K., Bebetsos, V., \& Douma, I. (2001). Self-talk in a basketball-shooting task. Perceptual and Motor Skills, 92(1), 309-315.

Theys, E. M. (2011, August 10). Leveling the playing field. Dance Magazine, Retrieved from http://dancemagazine.com/news/Leveling_the_Playing_Field/

Thomas, H., \& Tarr, J. (2009). Dancer's perceptions of pain and injury: Positive and negative effects. Journal of Dance Medicine \& Science, 13(2), 51-59.

Torres-McGehee, T. M., Green, J. M., Leaver-Dunn, D., Lepper, J. D., Bishop, P. A., \& Richardson, M. T. (2011). Perceptual and Motor Skills, 112(3), 711-725.

Turk, J. C., Prentice, W. E., Chappell, S., \& Shields, E. W. (1999). Collegiate coaches’ knowledge of eating disorders. Journal of Athletic Training, 34(1), 19-24. 
Turner, B. S., \& Wainwright, S. P. (2003). Corps de ballet: The case of the injured ballet dancer. Sociology of Health \& Illness, 25(4), 269-288.

Unruh, A. M., \& Ritchie, J. A. (1998). Development of the Pain Appraisal Inventory: Psychometric properties. Pain Research and Management, 3, 105-110.

Vealey, R. S., \& Greenleaf, C. A. (2001). Seeing is believing: Understanding and using imagery in sport. In J. M. Williams, (Ed.), Applied sport psychology: Personal growth to peak performance (pp. 247-283). Mountain View, CA.: Mayfield Publishing Co.

Wainwright, S. P., Williams, C., \& Turner, B. S. (2005). Fractured identities: Injury and the balletic body. Health, 9(1), 49-66.

Watson, E. C. (2013). Examining health correlates in an elite professional ballet company (Unpublished doctoral dissertation). The Adler School of Professional Psychology, Chicago, IL.

Weinberg, R., \& Gould, D. (2011). Foundations of sport and exercise psychology $\left(4^{\text {th }}\right.$ Ed.). Champaign, IL: Human Kinetics.

Wiese-Bjornstal, D. M., Smith, A. M., Shaffer, S. M., \& Morrey, M. A. (1998). An integrated model of response to injury: Psychological and sociological dimensions. Journal of Applied Sport Psychology, 10, 46-69.

Williams, J. M., \& Andersen, M. B. (1998). Psychosocial antecedents of sport injury: Review and critique of the stress and injury model. Journal of Applied Sport Psychology, 10(1), $5-25$.

Wilk, C., \& Turkoski, B. (2001). Progressive muscle relaxation in cardiac rehabilitation: A pilot study. Rehabilitation Nursing, 26(6), 238-243. 
Wyon, M. (2007). Testing an aesthetic athlete: Contemporary dance and classical ballet dancers. In E. M. Winter, A. M. Jones, R. C. R. Davison, P. D. Bromley, \& T. H. Mercer (Eds.), Sport and exercise physiology testing guidelines: The British Association for Sport and Exercise Sciences guide. New York, NY: Routledge.

Yatabe, K., Yui, N., Kasuya, S., Fujiya, H., Tateishi, K., Terawaki, F., Yoshida, A., Yoshioka, H., Terauchi, K., Miyano, H., Oyama, T., \& Musha, H. (2014). Anxiety and mood among ballet dancers: A pilot study on effects of a medical approach involving periodic intervention. Annals of Sports Medicine and Research, 1(1), 1001-1009. 


\section{Appendix B: Basic Demographics Survey}

1. Age

2. Sex (circle one)
Male
Female
Prefer not to report

3. Year in School (circle one)

Freshman/First-year

Sophomore

Junior

Senior

4. Total number of years in dance (at any level):

5. Current hours of dance training per week (on average):

6. Did you have to audition in order to enter into your dance program? Yes No

7. Please indicate the emphasis of your dance program (circle one or more):
a. Ballet based
b. Modern based
c. Mixed repertoire
d. Other (please specify):

8a. Are you currently injured as a result of dance? Yes No

$8 \mathrm{~b}$. If yes, what is your current injury or injuries?

9. Are you currently seeing a physician, physical therapist, or athletic trainer to treat a new or recurring injury? Yes No

10. Are you currently experiencing physical pain when you dance? Yes No

11. How often do you typically engage in exercise outside of dancing? (circle one):
a. Never
b. Occasionally
c. 1 or 2 days a week
d. 3-5 days a week
e. 6 or 7 days a week
f. More than once daily, 7 days a week 
12. Have you ever worked with a sport psychology consultant individually or in a group setting?

Yes No

13a. Are you familiar with deep breathing, or diaphragmatic breathing?

Yes No

13b. Please rate your familiarity with deep breathing, or diaphragmatic breathing (circle one):

Not familiar

1
Slightly familiar

2

\begin{abstract}
Fairly Familiar
\end{abstract}
3
Very Familiar

4

14a. Are you familiar with progressive muscle relaxation?

Yes No

14b. Please rate your familiarity with progressive muscle relaxation (circle one):

Not familiar

1
Slightly familiar 2
Fairly Familiar

3
Very Familiar

4

15a. Are you familiar with imagery, a mental skill for performance and/or relaxation? Yes No

15b. Please rate your familiarity with imagery, a mental skill for performance and/or relaxation (circle one):

Not familiar

1
Slightly familiar

2
Fairly Familiar

3
Very Familiar

4 
16a. Are you familiar with cognitive behavioral therapy, thought re-framing, or self-talk? Yes No

16b. Please rate your familiarity with cognitive behavioral therapy, thought re-framing, or self-talk (circle one):

Not familiar 1
Slightly familiar 2
Fairly Familiar

3
Very Familiar

4

17a. Are you familiar with mindfulness, meditation, and/or acceptance and commitment therapy?

Yes No

17b. Please rate your familiarity with mindfulness, meditation, and/or acceptance and commitment therapy (circle one):

Not familiar

1
Slightly familiar

2
Fairly Familiar

3
Very Familiar

4 


\section{Appendix C: Athletic Coping Skills Inventory-28}

Directions: A number of statements that athletes have used to describe their experiences are given below. Please read each statement carefully and then recall as accurately as possible how often you experience the same thing. There are no right or wrong answers. Do not spend too much time on any one statement.

\section{0 $=$ Almost Never $\quad 1=$ Sometimes $\quad 2=$ Often $\quad 3=$ Almost Always}

1. On a daily or weekly basis, I set very specific goals for myself that guide what I do.

$\begin{array}{llll}0 & 1 & 2 & 3\end{array}$

2. I get the most out of my talent and skills.

$\begin{array}{llll}0 & 1 & 2 & 3\end{array}$

3. When an instructor or choreographer tells me how to correct a mistake I've made, I tend to take it personally and feel upset.

$\begin{array}{llll}0 & 1 & 2 & 3\end{array}$

4. When I am dancing, I can focus my attention and block out distractions.

$\begin{array}{llll}0 & 1 & 2 & 3\end{array}$

5. I remain positive and enthusiastic during performances, no matter how badly things are going.

$\begin{array}{llll}0 & 1 & 2 & 3\end{array}$

6. I tend to perform better under pressure because I think more clearly.
0
1
2
3

7. I worry quite a bit about what others think about my performance.
0
1
2
3

8. I tend to do lots of planning about how to reach my goals.
0
1
2
3

9. I feel confident that I will perform well. 
0 $=$ Almost Never $\quad 1=$ Sometimes $\quad 2=$ Often $\quad 3=$ Almost Always

10. When an instructor or choreographer criticizes me, I become upset rather than helped.

0

12

3

11. It is easy for me to keep distracting thoughts from interfering with something I am watching or listening to.

$\begin{array}{llll}0 & 1 & 2 & 3\end{array}$

12. I put a lot of pressure on myself by worrying how I will perform.

0

1

2

3

13. I set my own performance goals for each practice/rehearsal.

0

1

2

3

14. I don't have to be pushed to practice/rehearse or perform hard; I give 100\%.

0

1

2

3

15. If an instructor or choreographer criticizes or yells at me, I correct the mistake without getting upset about it.

$\begin{array}{llll}0 & 1 & 2 & 3\end{array}$

16. I handle unexpected situations in dance very well.

$\begin{array}{llll}0 & 1 & 2 & 3\end{array}$

17. When things are going badly, I tell myself to keep calm, and this works for me.
0
1
2
3

18. The more pressure there is during a performance, the more I enjoy it.

0

1

2

3

19. While performing, I worry about making mistakes or failing to come through.

$\begin{array}{llll}0 & 1 & 2 & 3\end{array}$

20. I have my own game plan worked out in my head long before the performance begins. 
0 $=$ Almost Never $\quad 1=$ Sometimes $\quad 2=$ Often $\quad 3=$ Almost Always

21. When I feel myself getting too tense, I can quickly relax my body and calm myself. $\begin{array}{llll}0 & 1 & 2 & 3\end{array}$

22. To me, pressure situations are challenges that I welcome.

0

3

23. I think about and imagine what will happen if I fail or screw up.

0

1

2

3

24. I maintain emotional control no matter how things are going for me.

$\begin{array}{llll}0 & 1 & 2 & 3\end{array}$

25. It is easy for me to direct my attention and focus on a single object or person.

0

1

2

3

26. When I fail to reach my goals, it makes me try even harder.
0
1
2
3

27. I improve my skills by listening carefully to advice and instruction from instructors and choreographers.

$\begin{array}{llll}0 & 1 & 2 & 3\end{array}$

28. I make fewer mistakes when the pressure's on because I concentrate better.

$\begin{array}{llll}0 & 1 & 2 & 3\end{array}$

29. Have you responded to all of the above questions honestly?

Yes No 


\section{Appendix D: Pain Appraisal Inventory}

Directions: Please respond to the statements below about any troublesome physical pain that you have experienced in the previous two weeks:

1 = strongly disagree

$2=$ moderately disagree

$3=$ slightly disagree

$4=$ slightly agree

$5=$ moderately agree

$6=$ strongly agree

1. I am concerned that the pain might mean something is wrong with me.

$\begin{array}{llllll}1 & 2 & 3 & 4 & 5 & 6\end{array}$

2. I am concerned that the pain might become more than I can manage.

$\begin{array}{llllll}1 & 2 & 3 & 4 & 5 & 6\end{array}$

3. I am worried about getting things done.

$\begin{array}{llllll}1 & 2 & 3 & 4 & 5 & 6\end{array}$

4. I am concerned about how much more pain I can take.

$\begin{array}{lllllll}1 & 2 & 3 & 4 & 5 & 6\end{array}$

5. The pain seems threatening.

$\begin{array}{llllll}1 & 2 & 3 & 4 & 5 & 6\end{array}$

6. I am worried about being depressed or discouraged because of the pain.

$\begin{array}{llllll}1 & 2 & 3 & 4 & 5 & 6\end{array}$

7. I feel controlled by the pain.

$\begin{array}{llllll}1 & 2 & 3 & 4 & 5 & 6\end{array}$

8. I think of this pain as a threat.

$\begin{array}{llllll}1 & 2 & 3 & 4 & 5 & 6\end{array}$


1 = strongly disagree

2 = moderately disagree

3 = slightly disagree

4 = slightly agree

5 = moderately agree

6 = strongly agree

9. I think the pain is a chance to prove myself.

$\begin{array}{llllll}1 & 2 & 3 & 4 & 5 & 6\end{array}$

10. I think the pain is a test of my strength and ability.

$\begin{array}{llllll}1 & 2 & 3 & 4 & 5 & 6\end{array}$

11. I think that something good might come out of having the pain.

$\begin{array}{llllll}1 & 2 & 3 & 4 & 5 & 6\end{array}$

12. I think the pain makes me a stronger person.

$\begin{array}{llllll}1 & 2 & 3 & 4 & 5 & 6\end{array}$

13. I think the pain is a chance to learn more about myself.

$\begin{array}{llllll}1 & 2 & 3 & 4 & 5 & 6\end{array}$

14. I think that without this pain, there is no gain.

$$
\begin{array}{llllll}
1 & 2 & 3 & 4 & 5 & 6
\end{array}
$$

15. I think of this pain as a challenge.

$\begin{array}{llllll}1 & 2 & 3 & 4 & 5 & 6\end{array}$

16. I think the pain tests how well I can manage.

$\begin{array}{llllll}1 & 2 & 3 & 4 & 5 & 6\end{array}$




\section{Appendix E: Injury Tracking Survey}

This survey will ask you a series of questions about any injury or injuries that you may have experienced in the past week. Please think back on the past 7 days when answering these questions. (If a question does not apply to you, you may leave it blank or write N/A).

1. In the past week, have you experienced one or more injuries as a result of dancing?

$$
\text { Yes No }
$$

2. If so, please list the injury or injuries here:

3. Please place an "X" over the dot next to the area(s) of the body that apply to your injury(ies):

o Head/face

o Neck/cervical spine

o Shoulder/clavicula

o Upper arm

o Elbow

o Forearm

o Wrist

o Hand/finger/thumb

o Sternum/ribs/upper back

o Abdomen

o Lower back/pelvis/sacrum

o Hip/groin

o Thigh

o Knee

o Lower leg/Achilles tendon

o Ankle

o Foot/toe

o Other (please specify): 
4. Where did this injury or injuries occur? (Please place an "X" over one or more dots):

o In class

o In rehearsal

o In performance

o Cross-training

o Stretching

o Other (please specify):

5. Did you seek help or treatment from a medical professional (athletic trainer, physical therapist, physician, orthopedist, or other health care professional)?

Yes No

6. Did you have to miss any dancing as a result?

Yes No

7. If so, please list the estimated numbers below:

a. Number of dance classes missed this past week:

b. Number of rehearsals and/or performances missed this past week:

c. Total number of hours of dancing missed this past week:

8. In the past week, did you have to modify any of your training (such as completing fewer repetitions, only doing barre work, or cross-training instead of rehearsing)?

Yes $\quad$ No

9. If you modified any of your training in the past week, please describe the parts of class(es) or performance(s) in which you did and did not participate:

10. In the past week, did you dance while experiencing physical pain?

Yes No 
11. If in the past week you danced while experiencing physical pain, please rate your level of pain by circling the appropriate number:
Minor pain
Moderate pain
Severe pain
1
2
3

12. For the next several weeks, you will be asked to complete this Injury Tracking Survey online on a weekly basis. The research team will send you a survey link either via text or email. In order to protect your identity when completing this web-based survey, please create and then remember your code name for the duration of this study ( 2 letters followed by 4 numbers):

Example: AB1234 


\section{Appendix F: Dance Experiences Survey}

Dance-specific stressors are challenges, hardships, conflicts, or other demands that people tend to experience in their role as dancers.

\section{Directions:}

1. Circle "Y" (yes) if the stressor is applicable to you or to your dance activity and "N" (no) if the stressor is not applicable to you at the present time.

2. For every item that is applicable to you (circled "Y"), circle the number that best describes the impact that each stressor has upon you at the present time, where $\mathbf{- 3}$ represents "extremely negative" and +3 represents "extremely positive."

You do not need to rate the impact of those items that do not apply to you (circled "N").

Examples: Suppose you have been selected for an important dance event (i.e., a major concert) and you have also changed teachers.

1. Selected for an important event

$\begin{array}{llllllllll}\mathbf{Y} & \mathrm{N} & -3 & -2 & -1 & 0 & +1 & +2 & +3\end{array}$

2. Change of teacher

$\begin{array}{lllllllll}\mathbf{Y} & \mathrm{N} & -3 & \mathbf{- 2} & -1 & 0 & +1 & +2 & +3\end{array}$

3. Incurring a major injury

$\begin{array}{llllllllll}\mathrm{Y} & \mathbf{N} & -3 & -2 & -1 & 0 & +1 & +2 & +3\end{array}$

For Item 1, "Y" is circled (bolded) because it is applicable to you. You might assess the impact as very positive because acceptance in an important concert represents the satisfaction of a major goal.

For Item 2, "Y" is circled (bolded) because it is applicable to you. You might assess the impact as moderately negative. Even if the change is the best move in the long run, it may be negative at the present time if it involves a period of adjustment, unfamiliarity, and lack of closeness.

For Item 3, "N" is circled (bolded) because it is not applicable to you, as you do not have an acute injury at the present time.

See Questions on the Following Pages 
1. Relationship with teachers

$\begin{array}{lllllllll}\mathrm{Y} & \mathrm{N} & -3 & -2 & -1 & 0 & +1 & +2 & +3\end{array}$

2. Relationship with choreographers

$\begin{array}{lllllllll}\mathrm{Y} & \mathrm{N} & -3 & -2 & -1 & 0 & +1 & +2 & +3\end{array}$

3. Relationship with other dancers

$\begin{array}{lllllllll}\mathrm{Y} & \mathrm{N} & -3 & -2 & -1 & 0 & +1 & +2 & +3\end{array}$

4. Performance not meeting your expectations

$\begin{array}{lllllllll}\mathrm{Y} & \mathrm{N} & -3 & -2 & -1 & 0 & +1 & +2 & +3\end{array}$

5. Incurring an acute injury

$\begin{array}{lllllllll}\mathrm{Y} & \mathrm{N} & -3 & -2 & -1 & 0 & +1 & +2 & +3\end{array}$

6. Managing time (between training, school, work, family, friends, etc.)

$\begin{array}{lllllllll}\mathrm{Y} & \mathrm{N} & -3 & -2 & -1 & 0 & +1 & +2 & +3\end{array}$

7. Not being selected as a dancer

$\begin{array}{lllllllll}\mathrm{Y} & \mathrm{N} & -3 & -2 & -1 & 0 & +1 & +2 & +3\end{array}$

8. Performing above your expectations

$\begin{array}{lllllllll}\mathrm{Y} & \mathrm{N} & -3 & -2 & -1 & 0 & +1 & +2 & +3\end{array}$

9. Training conditions (space, equipment, etc.)

$\begin{array}{lllllllll}\mathrm{Y} & \mathrm{N} & -3 & -2 & -1 & 0 & +1 & +2 & +3\end{array}$

10. Unmet goals

$\begin{array}{lllllllll}\mathrm{Y} & \mathrm{N} & -3 & -2 & -1 & 0 & +1 & +2 & +3\end{array}$

11. Thinking of changing field of study

$\begin{array}{lllllllll}\mathrm{Y} & \mathrm{N} & -3 & -2 & -1 & 0 & +1 & +2 & +3\end{array}$


12. Traveling

$\begin{array}{lllllllll}\mathrm{Y} & \mathrm{N} & -3 & -2 & -1 & 0 & +1 & +2 & +3\end{array}$

13. Company or class cohesion

$\begin{array}{lllllllll}\mathrm{Y} & \mathrm{N} & -3 & -2 & -1 & 0 & +1 & +2 & +3\end{array}$

14. Use of performance-enhancing drugs by yourself or other dancers

$\begin{array}{lllllllll}\mathrm{Y} & \mathrm{N} & -3 & -2 & -1 & 0 & +1 & +2 & +3\end{array}$

15. Publicity and media coverage

$\begin{array}{lllllllll}\mathrm{Y} & \mathrm{N} & -3 & -2 & -1 & 0 & +1 & +2 & +3\end{array}$

16. Satisfying all goals

$\begin{array}{lllllllll}\mathrm{Y} & \mathrm{N} & -3 & -2 & -1 & 0 & +1 & +2 & +3\end{array}$

17. Number of performances per year

$\begin{array}{lllllllll}\mathrm{Y} & \mathrm{N} & -3 & -2 & -1 & 0 & +1 & +2 & +3\end{array}$

18. Physical demands of training

$\begin{array}{lllllllll}\mathrm{Y} & \mathrm{N} & -3 & -2 & -1 & 0 & +1 & +2 & +3\end{array}$

19. Psychological demands of training

$\begin{array}{lllllllll}\mathrm{Y} & \mathrm{N} & -3 & -2 & -1 & 0 & +1 & +2 & +3\end{array}$

20. Support from others (family, peers, girlfriend/boyfriend)

$\begin{array}{lllllllll}\mathrm{Y} & \mathrm{N} & -3 & -2 & -1 & 0 & +1 & +2 & +3\end{array}$

21. "Politics" associated with dance activities

$\begin{array}{lllllllll}\mathrm{Y} & \mathrm{N} & -3 & -2 & -1 & 0 & +1 & +2 & +3\end{array}$

22. Financial status

$\begin{array}{lllllllll}\mathrm{Y} & \mathrm{N} & -3 & -2 & -1 & 0 & +1 & +2 & +3\end{array}$ 
23. Level and consistency of motivation

$\begin{array}{lllllllll}\mathrm{Y} & \mathrm{N} & -3 & -2 & -1 & 0 & +1 & +2 & +3\end{array}$

24. Important upcoming performance

$\begin{array}{lllllllll}\mathrm{Y} & \mathrm{N} & -3 & -2 & -1 & 0 & +1 & +2 & +3\end{array}$

25. Selected for important concert or trip

$\begin{array}{lllllllll}\mathrm{Y} & \mathrm{N} & -3 & -2 & -1 & 0 & +1 & +2 & +3\end{array}$

26. Physical characteristics

$\begin{array}{lllllllll}\mathrm{Y} & \mathrm{N} & -3 & -2 & -1 & 0 & +1 & +2 & +3\end{array}$

27. Feedback from teachers

$\begin{array}{lllllllll}\mathrm{Y} & \mathrm{N} & -3 & -2 & -1 & 0 & +1 & +2 & +3\end{array}$

28. Feedback from choreographers

$\begin{array}{llllllllll}\mathrm{Y} & \mathrm{N} & -3 & -2 & -1 & 0 & +1 & +2 & +3\end{array}$

29. Feedback from other dancers

$\begin{array}{lllllllll}\mathrm{Y} & \mathrm{N} & -3 & -2 & -1 & 0 & +1 & +2 & +3\end{array}$

30. Physical preparation for next performance

$\begin{array}{lllllllll}\mathrm{Y} & \mathrm{N} & -3 & -2 & -1 & 0 & +1 & +2 & +3\end{array}$

31. Psychological readiness for next performance

$\begin{array}{lllllllll}\mathrm{Y} & \mathrm{N} & -3 & -2 & -1 & 0 & +1 & +2 & +3\end{array}$

32. Weight control

$\begin{array}{lllllllll}\mathrm{Y} & \mathrm{N} & -3 & -2 & -1 & 0 & +1 & +2 & +3\end{array}$

33. Having a minor, chronic injury

$\begin{array}{llllllllll}\mathrm{Y} & \mathrm{N} & -3 & -2 & -1 & 0 & +1 & +2 & +3\end{array}$


34. General frame of mind (mood)

$\begin{array}{lllllllll}\mathrm{Y} & \mathrm{N} & -3 & -2 & -1 & 0 & +1 & +2 & +3\end{array}$

35. Quality of sleep

$\begin{array}{lllllllll}\mathrm{Y} & \mathrm{N} & -3 & -2 & -1 & 0 & +1 & +2 & +3\end{array}$

36. Relationships between faculty

$\begin{array}{lllllllll}\mathrm{Y} & \mathrm{N} & -3 & -2 & -1 & 0 & +1 & +2 & +3\end{array}$

37. Diet

$\begin{array}{lllllllll}\mathrm{Y} & \mathrm{N} & -3 & -2 & -1 & 0 & +1 & +2 & +3\end{array}$

38. Choreographer's expectations

$\begin{array}{lllllllll}\mathrm{Y} & \mathrm{N} & -3 & -2 & -1 & 0 & +1 & +2 & +3\end{array}$

39. Teacher's expectations

$\begin{array}{lllllllll}\mathrm{Y} & \mathrm{N} & -3 & -2 & -1 & 0 & +1 & +2 & +3\end{array}$

40. Family or friend's expectations

$\begin{array}{lllllllll}\mathrm{Y} & \mathrm{N} & -3 & -2 & -1 & 0 & +1 & +2 & +3\end{array}$

41. Other dancers' expectations

$\begin{array}{lllllllll}\mathrm{Y} & \mathrm{N} & -3 & -2 & -1 & 0 & +1 & +2 & +3\end{array}$

42. Performing

$\begin{array}{lllllllll}\mathrm{Y} & \mathrm{N} & -3 & -2 & -1 & 0 & +1 & +2 & +3\end{array}$

43. Choreographer's behavior during performances

$\begin{array}{lllllllll}\mathrm{Y} & \mathrm{N} & -3 & -2 & -1 & 0 & +1 & +2 & +3\end{array}$

44. Change of teacher

$\begin{array}{llllllllll}\mathrm{Y} & \mathrm{N} & -3 & -2 & -1 & 0 & +1 & +2 & +3\end{array}$


45. General health (colds, flu, etc.)

$\begin{array}{lllllllll}\mathrm{Y} & \mathrm{N} & -3 & -2 & -1 & 0 & +1 & +2 & +3\end{array}$

46. Fear of getting injured

$\begin{array}{lllllllll}\mathrm{Y} & \mathrm{N} & -3 & -2 & -1 & 0 & +1 & +2 & +3\end{array}$

47. Fear of failing grades

$\begin{array}{lllllllll}\mathrm{Y} & \mathrm{N} & -3 & -2 & -1 & 0 & +1 & +2 & +3\end{array}$

48. Fear of being replaced by another dancer

$\begin{array}{lllllllll}\mathrm{Y} & \mathrm{N} & -3 & -2 & -1 & 0 & +1 & +2 & +3\end{array}$

Please list and rate any other experiences that apply to you at the present time but are not on the above list:

49.

$\begin{array}{lllllllll}\mathrm{Y} & \mathrm{N} & -3 & -2 & -1 & 0 & +1 & +2 & +3\end{array}$

50.

$\begin{array}{lllllllll}\mathrm{Y} & \mathrm{N} & -3 & -2 & -1 & 0 & +1 & +2 & +3\end{array}$

51.

$\begin{array}{lllllllll}\mathrm{Y} & \mathrm{N} & -3 & -2 & -1 & 0 & +1 & +2 & +3\end{array}$

52.

$\begin{array}{lllllllll}\mathrm{Y} & \mathrm{N} & -3 & -2 & -1 & 0 & +1 & +2 & +3\end{array}$

53.

$\begin{array}{lllllllll}\mathrm{Y} & \mathrm{N} & -3 & -2 & -1 & 0 & +1 & +2 & +3\end{array}$

54.

$\begin{array}{lllllllll}\mathrm{Y} & \mathrm{N} & -3 & -2 & -1 & 0 & +1 & +2 & +3\end{array}$




\section{Appendix G: Intervention Protocol Outline}

1. First session (week one)

a. Personal introduction

i. Introduction to the primary researcher and research assistant

ii. Researcher's background in dance

iii. Researcher's current studies

iv. Overview of schedule of sessions

b. Brief program overview

i. Provide overview of each week's topic

ii. Provide context/background for the topics

c. Informed consent

i. Inform participants of their rights

ii. Statement on attendance and grading

iii. Gather informed consent forms

d. Administer instruments

i. Give clear directions, Hand out pencils/pens

ii. Collect all instruments

2. Second session (week two)

a. Overview of deep/diaphragmatic breathing and progressive muscle relaxation

i. Definitions

ii. Common uses

b. Example scripts (activities)

i. Conduct in-session deep breathing exercise

ii. Process participants' reactions to the exercise

iii. Conduct in-session PMR exercise

iv. Process participants' reactions to the exercise

3. Third session (week three)

a. Overview of imagery

i. Definitions

ii. Common uses and types

b. Example imagery script: Performance imagery

i. Conduct in-session Imagery script

ii. Process participants' responses

c. Example imagery script: Relaxation and Coping imagery

i. Conduct in-session Imagery script

ii. Process participants' responses

4. Fourth session (week four)

a. Overview of cognitive restructuring/self-talk

i. "You can't change your thoughts unless you're aware of them!"

ii. Introduction to the $\mathrm{ABC}$ model of thoughts (CBT/REBT) 
b. Seek feedback

i. What do participants already know about thoughts cause feelings?

ii. How important is attitude and self-confidence in dance?

iii. In what ways is this easy or hard in training or performances?

c. Worksheet activity

i. Facilitate small group activity

ii. Have participants complete worksheet together

iii. Circulate for questions, comments, on-task evaluation

iv. Process activity with large group

5. Fifth session (week five)

a. Overview of mindfulness

i. Definitions

ii. Common uses

b. Gather participants' knowledge

i. How are they currently using it? If so, is it working?

ii. In what contexts can they see themselves using it?

iii. What concerns or questions do they have?

c. Conduct mindfulness exercise

i. In-session exercise using an object (i.e., rock) to focus

ii. Provide step-by-step directions following script

iii. Process the experience in large group format

6. Sixth session (week six)

a. Brief overview of topics covered

i. Deep breathing, Progressive muscle relaxation

ii. Imagery (for performance, relaxation, and coping)

iii. Self-talk (from a cognitive-behavioral perspective)

iv. Mindfulness

b. Primary researcher explains final data collection

i. Reviews logistics and reintroduces research assistant

ii. Thank the participants for their voluntary participation

iii. Remains available after session for questions

c. Administration of instruments

i. Give clear directions, Hand out pencils/pens

ii. Collect all instruments

7. Focus Group Discussion (occurred same day as sixth session)

a. Research assistant explains procedure

i. Reminds everyone about voluntary participation

ii. Informs participants of the audio-taping equipment

b. Conducts discussion using the interview guide

i. Thank-you message to everyone who participated 


\section{Appendix H: Intervention Program Exercises and Activities}

\section{Deep Breathing Exercise Script}

Please prepare to begin this exercise by lying down on the floor, or if you prefer, sitting in a comfortable position. You may choose to close your eyes as well, to focus your attention on your breath.

As we begin this deep breathing exercise, gently close your eyes and begin to channel your energy towards your breath. Place your hand over your abdomen, just above where you might put on a belt. Take a deep breath in, and feel this breath go into your belly. Hold it for a moment, and then exhale, hearing the air whoosh out through your mouth.

When you're ready, take another deep breath in. Feel your belly rise slowly as the air enters your body. Let it out with another whooshing sound, like the wind, as you blow the air out from your mouth. Each breath leaves you more and more relaxed. Each breath relaxes your whole mind and body.

Take another deep breath down into your belly. Feel your belly expand. And as you relax and exhale, imagine any tension that you have leaving your body. Feel the breath cleaning your body of any stress or tension that you may have. As you continue to breathe at your own comfortable pace, relax and enjoy the feeling of peace and calmness that has spread throughout your body.

Now let your breathing slow down a bit and become automatic. Allow each breath to continue to relax you. You are breathing peacefully and calmly. You are breathing in an easy, natural way. Each time you exhale, silently say to yourself "one."

Continue to breathe in and out, saying "one" to yourself each time you exhale. Whenever thoughts or perceptions take your attention away from your breathing, simply let go of these thoughts or perceptions, and return to saying "one." Breathe naturally and calmly, silently saying "one" as you breathe.

And now, take three slow, deep breaths on your own time. Allow the fullness of these breaths to relax and calm you. And once you've completed those three breaths, allow your hands to fall gently from your belly down to the floor. Allow yourself to feel grounded in this room, in this space, as you prepare to end this breathing exercise.

As your hands fall gently to the floor, prepare to open your eyes and return to a seated position. On your own time, allow your eyes to open and move your body to a seated position. Once you are sitting on the floor, take one more deep breath to signify the end to this deep breathing exercise.

Please note that the researcher has adapted this script from the deep breathing exercise found here: http://www.dhs.state.mn.us/main/groups/disabilities/documents/pub/dhs id 059333 1.pdf 


\section{Active or Passive Progressive Muscle Relaxation Exercise Script}

Before beginning this exercise, scan your body to identify any areas of pain or injury that you're experiencing. Please be aware of these areas throughout this exercise. Ease up or stop if needed.

Progressive muscle relaxation exercises are exercises that involve the progressive tensing and relaxing of muscles or muscle groups. By tightening and then releasing the muscles, you can feel the difference between tensed and relaxed.

Throughout this exercise, make sure not to do any movements that cause pain. If any of these exercises causes discomfort, ease up or stop to ensure that you do not cause muscle cramping or injury. This is very important; there is no need to experience pain or injury as a result of this exercise. When in doubt, ease up or stop.

This guided progressive muscle relaxation exercise will begin now. Start by finding a comfortable position-- either sitting, standing, or lying down. You will probably find that sitting or lying down is the most comfortable position for this exercise. However, you can change positions any time to make yourself more comfortable as needed.

The first progressive muscle relaxation exercise is breathing. Breathe in deeply and hold this breath. Hold it, hold it, and now release. Let all of the air go out slowly and release all of the tension. Take another deep breath in, hold it, and then exhale slowly, allowing the tension to leave the body with the air. Now breathe in even more slowly, breathe in, hold, and out. Breathe in, hold, and out. Continue to breathe slowly and gently. Allow your breathing to relax you.

The next part of this exercise focuses on relaxing the muscles of your body. Start with the muscles of your feet. Tighten the muscles of your feet, tense the muscles even further, and hold onto this tension. Feel how tight and tensed the muscles in your feet are. Feel the muscles wanting to give up this tension, and continue to hold it. And now relax, let all the tension go. Feel the muscles in your feet going limp and loose, and relaxed. Notice how relaxed the muscles feel now. Notice the difference between tension and relaxation. Enjoy the pleasant feeling of relaxation in your feet.

Now focus on the muscles in your ankles. Tighten the muscles of your ankles, tense the muscles even further, and hold onto this tension. Feel how tight and tensed the muscles in your ankles are. Feel the muscles wanting to give up this tension. And now relax. Let all the tension go. Feel the muscles in your ankles going limp and loose, and relaxed. Notice how relaxed the muscles feel now. Notice the difference between tension and relaxation. Enjoy the pleasant feeling of relaxation in your ankles.

Now focus on the muscles of your calves and shins. Tighten the muscles of your calves and shins, tense the muscles even further, and hold onto this tension. Squeeze the calves and shin muscles harder, and hold it. And now, let all the tension go. Feel the muscles in your lower legs going limp and loose, and relaxed. Enjoy the pleasant feeling of relaxation in your calves and shins. 
Now focus on your upper legs-- your quadriceps and your hamstrings. Tighten these muscles, tighten them even further, and hold this tension. Squeeze these muscles harder, feeling the muscles wanting to give up tension. And then release-- letting all the tension go. Notice how relaxed the muscles of your quads and hamstrings feel now. Notice the difference between tension and relaxation in your upper legs.

And now focus again on your breathing. Take slow, even, regular breaths. Breathe in relaxation and breathe out any remaining tension. Breathe in relaxation, and breathe out tension. Continue to breathe slowly and rhythmically. Begin to focus on your buttocks. Tighten these muscles as much as you can. Hold this tension, and then release. Relax the muscles of your buttocks. Enjoy the pleasant feeling of relaxation.

Now focus on your stomach and your chest. Tighten these muscles further. Hold it... and then release. Relax all of these muscles. Enjoy the feeling of relaxation that you're experiencing in your stomach and chest.

Now bring your focus to your upper back and your shoulders. Tighten the muscles in your back and shoulders. Feel your back tightening, pulling your shoulders back and feeling the muscles come together at your spine. Arch your back if you feel the need to, and hold. And relax-- let all the tension go. Feel your back comfortably relaxing into a good and healthy posture, maintaining the natural curves of your spine.

Now focus on your upper arms-- your biceps, triceps, and elbows. Tighten the muscles of your upper arms, tense the muscles even further, and hold onto this tension. Feel how tight and tensed the muscles in your upper arms are. Hold it, hold it, and now relax, let all the tension go. Feel the muscles in your biceps, triceps, and elbows going limp and loose, and relaxed. Notice how relaxed the muscles feel now.

And now bring your focus to your wrists, hands, and fingers. Tighten your wrists by squeezing your hands into tight fists. Squeeze harder, harder. Hold the tension in your hands and fingers. Feel the tension in these muscles. Hold it for a few moments more... and now release. Let the muscles of your wrists, hands, and fingers relax and go limp. Feel the relaxation as your hands lower into a comfortable position and relax at your sides. Allow these muscles of your wrists and hands to relax completely.

Focus again on your breathing. Take slow, even, regular breaths. Breathe in relaxation and breathe out any remaining tension. Breathe in relaxation, and breathe out tension. Continue to breathe slowly and rhythmically.

Finally, bring your attention to your facial muscles-- your chin, cheeks, lips, nose, ears, and forehead. Bring your focus to all of the muscles that make up your face. Scrunch your eyes tightly, and tightening your cheeks and your chin. Hold these muscles as tightly as you can. And now relax. Release all of the tension. Feel how relaxed your face is.

Notice all of the muscles in your body. Notice how much more relaxed your muscles feel. Allow any last bits of tension to drain away. 
And now take a quick scan of the parts of your body that you've just relaxed, starting with your feet. Focus on the relaxation in your feet, and then move to your ankles. Then to your calves and shins, and to your quads and hamstrings. Then your buttocks, your stomach and chest. Then to your back and shoulders, your upper arms, your wrists and hands, and finally the muscles in your face.

Let your muscles become loose and limp and relaxed. Enjoy the relaxation you're experiencing. Notice your calm breathing, your relaxed muscles-- enjoy the relaxation for a few moments.

When you're ready to return to your usual level of awareness and alertness, slowly begin to awaken your body. Wiggle your toes and fingers, swing your arms gently, shrug your shoulders, or stretch if you like.

Allow your eyes to begin to take in your surroundings, and your ears to take in any noises you may hear around you.

And on your own time, continue to breathe and allow yourself to come to a seated position with your eyes open. You may now end this progressive muscle relaxation exercise feeling calm and refreshed.

Please note that this script was developed by the researcher, based on the PMR Exercise by Inner Health Studio found here: https://www.youtube.com/watch? $v=H F w C K K a--18$ 


\section{Class or Performance Imagery Script}

As you sit [or lie down] here and allow your eyes to close, begin to focus on your breathing. Notice how your breath moves in and out, and how your belly responds to each inhale and exhale. Feel your belly expand as you inhale and fall as you exhale. If you're having trouble focusing on your breathing, try to put less pressure on yourself and just lay your hands across your lower belly so that you can feel your body as it inhales and exhales.

And as you continue to breathe, imagine a typical day of dance class. Picture yourself walking into the studio. What are you wearing? Note the clothing you've chosen-- what colors, textures, and layers you've chosen.

Now turn your attention to your body. Notice that it's a relaxed and excited body that is ready to start dancing. See yourself beginning to lightly stretch out your muscles, as if inviting each muscle to prepare for dancing. Imagine yourself in a calm, collected, ready mindset.

And now turn your attention to your surroundings. Notice the lights above you, the floor below you, the barres and the mirrors around you. And as you notice them, silently thank them for being a part of your dancing today.

Also notice the sounds around you. Is there music playing? Can you hear your classmates talking as they prepare for class? Allow these sounds to reverberate in your ears, and to create the soundtrack of your day.

And picture class about to start, and sense that you're feeling excited to dance today. Your body is warming up and your heart rate is becoming slightly elevated. As you warm up your body for class, your movements are fluid and you feel ready to focus on each task that you're asked to perform. Your mind is at ease, and you've let go of all other worries as you focus on your dancing.

You are at ease with your dancing today, and dancing will be enjoyable for you. You are smiling inside and glad that you're dancing today. You decide to accept any challenges that come your way-- perhaps a difficult combination or an improvement in your posture. You choose to rely on your training and your focused attention to help you dance well. Your body remains warm as you execute each movement. Your excitement to be dancing today continues.

As you see yourself excited to be dancing, take a few deep breaths and notice your body's feelings of preparedness. Notice the sense of calmness and confidence in your body. Take three deep breaths, and with each breath remind yourself of your choice to dance confidently today.

And as you complete these deep breaths, begin to bring your awareness back to where your body is sitting [or lying] at this moment-- noticing what is beneath you and around you. Acknowledge the time you've spent helping to exercise your mind, and preparing it for class. And once you've come back to the present moment, you should feel free to open your eyes slowly and allow yourself to sit in silence [as others do the same]. Now take one final deep breath as you end this imagery exercise.

Please note that this script was developed by the primary researcher, based on the recommendations of Leslie-Toogood, Hammond, and Gregg. See this link for more information: http://www.academia.edu/4256624/How to develop your own imagery scripts Developed by Dr. Adrienne Leslie-Toogood 


\section{Relaxation and/or Coping Imagery Script}

Making yourself comfortable lying down on the floor

Either keeping your legs straight or bending them comfortably at a right angle

Allow your eyes to close, your body to melt into the floor

Allow your attention to gently come into the body and the flowing of the breath

Experience the sense of the body as a whole, lying here, extended, and breathing

And when you feel ready, picturing in your mind's eye

The image of a tree

Perhaps a tree that you know well and frequent, or one that you've seen in a photograph, or even an imagined tree that you've created

Bring this tree into vivid focus in your mind's eye

Notice how it's a strongly rooted tree... a tall, thick tree held in the earth's surface with many complex roots-- perhaps being fed by a spring or a stream or some rich soil

And if you watch and observe the tree carefully

You'll notice that it's always changing,

And yet it's also always itself

It changes as the wind moves from north to south,

And as the light is reflected on the bark in different ways

It changes depending on the weather and the season

Sometimes on very calm days the tree stands very still

With great precision, as if it were trying to hide amongst the landscape

The clouds... the birds... the grasses that might surround the tree...

And there are times when the tree responds to its surroundings

And contains in a certain way everything that comes by, everything that passes high over it

And at different times the tree might be very active and quite pronounced in its swaying

And of course, the tree changes through the seasons, and in the winter, it might shed its leaves, or be layered with icicles and snow,

and as spring rolls in, the storms may test the tree's balance and deep rootedness,

with thunder and lightning passing by overhead

And so, through day and night and through the seasons and over the years,

The tree is always changing...

And yet it also always remains itself-- its own essential nature

And let's see if we can't invite the tree to become co-extensive with our own body

So that the tree becomes a part of us and we a part of the tree

And recognizing that at times our own minds are indeed like the tree

Our minds can be reflective like the tree, at times swaying back and forth, and at other times

things can be very still

And getting in touch with the sense of the entirety of the tree,

Both at the surface and down below it

And as you lie here, just experience the entirety of your being

Recognizing that at times we may indeed feel that our minds are swaying or frozen

And during those times we can drop down beneath the surface of our minds' activity

And find that place of stillness and calmness and stability that is always there...

And is always available to us

With each moment, with each breath, and certainly when we lie down in this way 
Under the canopy of our own vast awareness, just experience The moment-to-moment changes in the body, the feelings in the body, acknowledging its sounds, its sensations...

And allowing your awareness to hold it all, moment-by-moment, breath-by-breath In your own fullness, in your own completeness, moment by moment, breath-by-breath... And as this relaxation exercise comes to a close, take one final deep breath And allow your eyes to slowly open as you bring your attention back to the present moment And your being in this space...

You may now end this relaxation exercise.

Please note that this script was developed by the primary researcher, based on the Lake Meditation and Mountain Meditation by Jon Kabat-Zinn. See this link for a sampling:

https://www.youtube.com/watch? $v=B z v \quad z X W I M T M$ 


\section{Self-Talk Small Group Exercise - Page 1}

Sample Initial Statements:

1) I give up. Today is such a waste.

2) If I get stressed out, I know I'll train/rehearse/perform poorly.

3) I really messed that up. There's no recovering from it.

4) I must prove myself to everyone each and every day.

5) If it weren't for the floor/music/choreography, my skills and talent would shine through.

6) If people see me taking it easy, they'll think I'm lazy.

7) This day/semester/rehearsal/feeling will never end.

8) I will never feel good about how my body looks/moves.

9) My instructor/collaborator/fellow dancer makes me crazy.

10) This audition will determine my future as a dancer.

11) My dancing is a direct measure of my worth.

12) Clearly, I am not as good as I thought.

\section{Self-Talk Small Group Exercise - Page 2}

Sample Revised Statements:

1)

2)

3)

4)

5)

6)

7)

8)

9)

10)

11)

12) 


\section{Mindfulness or Guided Sitting Meditation Script}

Please prepare to begin this exercise by sitting comfortably on the floor or in a chair. You may choose to use the wall or a pillow for additional support if sitting upright causes any tension, pain, or discomfort. We will soon begin a guided sitting meditation.

This exercise will introduce you to mindfulness, which is one strategy that you can use to cope with things that may be happening in your life. Just like you do many repetitions when you dance, mindfulness requires some practice. Do not worry about being perfect in this exercise; you are learning to train your brain to think differently than you may be asked to think in a lecture or seminar.

This meditation will help you learn to simply be and to look within yourself with mindfulness and awareness. There is no right or wrong way to do this guided exercise. Honor your own process, and try not to judge it. It is okay if you do not do exactly what I ask or describe. Consider this room a place of non-judgment as you begin this exercise.

Allow yourself to switch from the usual mode of doing to a mode of non-doing. A mode of simply being and observing. And as you allow your body to become still, bring your attention to the fact that you are breathing. And become aware of the movement of your breath as it comes into your body and as it leaves your body. There is no need to manipulate or change the breath in any way. Simply be aware of it and of the feelings associated with breathing.

Observe the breath deep down in your belly-- feel the belly as it expands gently on the inhale, and as it falls back towards your spine on the exhale. Being totally here in each moment with each breath. Not trying to do anything, not trying to get any place, simply being with your breath.

And as you continue to breathe in this way, bring your attention to the rock that you've chosen to hold in your hand. Really focus on the rock, bringing your mind and your eyes to the rock itself, and all of its features.

With your breath continuing, begin to notice with your hands the weight of this rock. Is it heavier than you thought? Lighter? Just about what you'd imagined it would be? Notice how holding the rock affects the shape of your palm.

And now notice the color of your rock. Is it earthy and brown, or bright and blue? Or is it some other color, perhaps a blend of colors? Noticing the weight and the color of your rock, continue to breathe with your belly.

And now, with your breath continuing, begin to notice with your hands the surface of this rock. Are there ridges or bumps on your rock? Or is it smooth?

Continue focusing on your breath and allow your attention to stay on the rock - its weight, its color, and its texture. And as you continue to breathe and to observe your rock, you may find that from time to time your mind will wander off into thoughts, fantasies, anticipations of the future 
or the past-- worrying, memories, whatever it may be. When you notice that your attention is no longer here and no longer with your breathing, and without judging yourself, bring your attention back to your breathing and ride the waves of your breathing, fully conscious of the duration of each breath from moment to moment.

Allow your focus to come back to your rock - to the one thing on which you are trying to focus as you breathe. Again, notice and appreciate the rock's features - it's texture, color, and weight. Its perfections or imperfections.

And every time you find your mind wandering off the breath and the rock, gently bring your attention back to the present, back to the moment-to-moment observing of the flow of your breath and the rock that you are holding in your hand. Use your breath and the rock to help you tune into a state of relaxed awareness and stillness.

And you might give yourself credit for having spent this time nourishing yourself in a deep way by dwelling in this state of non-doing, in this state of being, of breathing and observing. For having intentionally made time for yourself to simply be who you are - to breathe and to notice in a very personal, intentional way the rock that you have chosen. Acknowledge your breath and this rock, and the present moment.

And as this sitting meditation ends, and you move back into the world, come to notice more fully your surroundings, and allow the benefits of this practice to translate to every aspect of your life.

When you hear the tune [or music], I invite you to place your rock in front of where you are sitting and to begin to make eye contact with those around you.

Please note that this script was developed by the primary researcher, in collaboration with a licensed counselor and counseling educator. There are likely to be many similar scripts found online and in published books or journals focused on meditation, or mindfulness, practice. 


\section{Appendix I: Adherence Journal}

1. Please enter your code name (Two letters followed by four numbers):

2. Today's date:

3. Over the past week, what skills did you practice on your own time? (check all that apply)

o None

○ Progressive muscle relaxation

- Autogenic training

- Imagery (for performance, relaxation, or healing)

○ Cognitive reframing (self-talk, affirmations, etc.)

4. For how long did you practice these skills, in total?

○ 0 minutes

- 1-10 minutes

o 11-20 minutes

○ 21-30 minutes

○ 31-40 minutes

○ 41-50 minutes

- 51-60 minutes

○ $61+$ minutes

5. What materials, if any, did you use to assist you in practicing these skills? (check all that apply)

○ None

○ Videos

O Scripts

- Podcasts

○ Music

- A friend or classmate

o Other:

6. How would you rate your efforts to practice these skills?

○ 1 - No effort

○ 2 - Some effort

○ 3 - Good effort

○ 4 - Excellent effort

7. If there is anything you'd like to share about your understanding or practicing of these skills, materials used, or time spent, please write them here: 


\section{Appendix J: Participant Contact Information (Confidential)}

In order to receive emails or text messages pertinent to this study, please share the following information with the research team members.

Your contact information will not be shared with any other parties, and will not be used to identify you in any research publications.

This information will only be used to send you weekly survey links and/or reminders. This information may also be used to contact raffle winners (see explanation in Consent Form).

Phone Number:

Please note that you must have a smartphone in order to receive weekly texts from the research team. Data rates may apply.

Email Address:

You may choose to share a school or personal email address. Please be sure it is a current account to which you know the username and password. 


\section{Appendix K: Semi-Structured Interview Guide for Focus Group Discussion}

1) Reflecting back on the six-week intervention program in which you were a participant, what worked well for you?
a) Structure of sessions
b) Content of sessions
c) Relationship with intervention presenter
d) Materials used in sessions
e) Materials used for data collection purposes
f) Other

2) What did not work well for you?
a) Structure of sessions
b) Content of sessions
c) Relationship with intervention presenter
d) Materials used in sessions
e) Materials used for data collection purposes
f) Other

3) What information that was presented during this intervention program was new or surprising to you?

4) How do you see yourself using any or all of this information in the future?
a) Dance class
b) Rehearsal
c) Performance
d) Academics
e) Social life
f) Personal life
g) Other

5) What, if anything, would you recommend changing about this intervention program?

Please Note: Content displayed under each question is to be used when necessary to prompt further discussion. 


\section{Appendix L: Stakeholder Interview Findings}

The individual interviews with each dance class instructor consisted of discussion focused on four main topics: (1) their institution's and program's culture, along with their personal teaching philosophies, (2) their perceptions of the psychosocial demands of dance, (3) the role that they feel injury plays in the dance world/profession, and finally (4) the reason or reasons why they decided to express interest in the research study.

\section{Major Findings}

Description of the university and dance program. Major themes that the instructors used to describe their university environment, and specifically their dance program, included support and service; family; technical skills and instruction; and empowerment. In the following section, each theme is supported by relevant quotes taken from the interview transcriptions.

Support and service. The theme of supporting one another and providing service to those in the university community was apparent. For instance, one instructor stated that: "People are willing to help around here...[Y]ou don't just go standing in line for an hour waiting for someone to tell you to head down the hall to another office...it's not that kind of an institution." When it comes to serving the students directly, one instructor stated that her goal is to "look at the culture of our students and see what areas they are missing and in what ways can I support them in their process."

Family. When asked to describe their dance program, one instructor said: "We have a very tight-knit often dysfunctional group...it's like a big giant family and sometimes people agree and sometimes they don't and...it moves along that way."

Technical skills and instruction. Both instructors stated that a focus on technical skill was important to understanding their program's environ: "Because they [the students] have the strong ballet component...they know how to stand on their feet and get off their feet and all that stuff." And because the program is ballet-based, one instructor said, "the students are...technically more versatile than students in other universities that just have other techniques and very little ballet."

Empowerment. Along with the themes of family and support, the instructors indicated that empowering students to figure out what they have to offer to others and what drives their careers is important: "[The students should] take what I've tried to teach you and go out there and go for it." 
Psychosocial demands of dancers. The instructors were asked to elaborate on any psychosocial demands that they perceived their students to experience. Major themes included body aesthetics (including body shape and size); stereotypes of dancers; the importance of selfcare; professional development; and maintaining a solid work ethic.

Body aesthetics. According to one instructor, "Classical ballet has a look, and that's the way that it is." The instructor explained that there is little room for deviation from the preferred or ideal body size and shape that ballet has historically required of those who are successful at a high level of performance.

Stereotypes. "Unfortunately, there's a stigma...that artists are kind of flaky and you know unreliable and kind of just out there." The instructor indicated that this stigma can be hurtful to the dancer if it is not true to the individual him/herself.

Self-care. One instructor indicated that self-care is essential for a successful dancer in the long-term, and emphasized healthy eating and a manageable schedule: "As advisors we try to make sure that they have scheduled lunch for themselves...that they have a plan of how they're going to be eating and caring for themselves."

Professional development. The instructors also discussed the need for their students to find their 'place' in the dance world and to present themselves as professionals upon graduation: "We have [a class] at the very end of their journey before they go off into the world...so they have portfolios that they turn in...they are required to get one letter of recommendation so that they keep building their portfolios"

Work ethic. Lastly, one instructor claimed that "a strong work ethic is often worth more than super fabulous technique," noting that hard work, accountability, and dedication should be praised in addition to technical skill or inherent talent.

Injury in dance. When asked about the role that injury plays in the dance world, and specifically in their students' training and performance experiences, the instructors cited prevention as key to the injury process. They also discussed several antecedents to an injury, and the fact that in some cases, injuries are just accidents that we cannot predict.

Prevention. With respect to preventing injuries, one instructor stated that prevention is about "understanding one's own body...you know I like to think that is all gets down to a kinesthetic awareness." Self-care was also noted here, as "it becomes extremely important to utilize all of the tools you've been learning about...time management, self-care, and that you're 
not putting yourself at greater risk." Lastly, there was an emphasis on appropriate training and execution of technical skills: "[M] any injuries can be avoided if people are trained properly."

Antecedents. Two antecedent factors to injury were discussed. The first was stress, especially at the end of the semester: "There's lots of culminating stuff...projects and papers that might be happening." The second factor was training or performing in times of stress, illness, or pain when one should be resting, tapering, or being a better listener to the body "There's problems if you kind of push through it as well."

Accidents. Finally, one instructor indicated that while she did not want to say that injuries are an inevitable part of dancing, "I think that accidents certainly happen and that's just kind of the name of the game [in dance]."

Reasons for allowing classroom access to the primary researcher. Both instructors were asked to report their reason(s) for allowing the primary researcher access to their respective classrooms in order to recruit volunteer participants for the purpose of the study. The instructors indicated three main reasons, namely time, intervention program content, and the students' experience as part of a research project.

Time. One instructor said that, "[I] n all honesty to have...class periods taken away from me was just fine...[I] was out of [new] material." This instructor explained that she was ahead of schedule with her students, and so the researcher's visits were rather welcomed.

Content. "Dancers are very hard on themselves...they don't always cultivate the kind of self-care that they need to," said one instructor. From her perspective, even if she had to sacrifice a bit of time or class content to accommodate for the researcher's visit, it was important to show the students ways to engage in self-care that they might otherwise overlook or deem trivial.

Student experience. Both instructors emphasized that their classrooms are fairly studentcentered and experience-focused. "We're more empowered by sharing our experiences...in general as a population I think probably needs more of this than they think." One instructor elaborated, "I was interested in seeing how my students reacted to such a thing...to watch my own students go through the process and see what they got out of it." For her, there was value in watching her students engage in the research process and gain an understanding of how they can contribute to scientific inquiry. According to this instructor, many students were curious about the process and seemed eager to be involved. 


\section{Appendix M: Sample Self-Talk Statements and Associated Bible Verses}

1. I give up. Today is such a waste.

"Do not be afraid, for I am with you. Do not be dismayed, for I am your God. I will strengthen you. I will help you. I will uphold you with my victorious right hand." (Isaiah 41:10)

"Jesus said, In this world you will have trouble. But take heart, I have overcome the world." (John 16:33)

2. If I get stressed out, I know I'll train/rehearse/perform poorly.

'Those who trust in the Lord are like Mount Zion, which cannot be shaken but endures forever." (Psalm 125:1)

"When I said, 'My foot is slipping,' Your love, O Lord, supported me. When anxiety was great within me, Your consolation brought joy to my soul." (Psalm 94:18-19)

3. I really messed that up. There's no recovering from it.

"Be still, and know that I am God." (Psalm 46:10)

"In Him all things hold together." (Colossians 1:17)

"As far as the east is from the west, so far He removes our transgressions from us." (Psalm 103:12)

4. I must prove myself to everyone each and every day.

"Your mercies are made new every morning." (Lamentations 3:23)

"God is faithful. He will not let you be tempted beyond what you can bear. But when you are tempted, He will also provide a way out so that you will be able to endure it." (1 Corinthians $10: 13)$

5. If it weren't for the floor/music/choreography, my skills and talent would shine through. "Whatever you do, work at it with all your heart, as working for the Lord, not for human masters, since you know that you will receive an inheritance from the Lord as a reward. It is the Lord Christ you are serving." (Colossians 3:23-24)

6. If people see me taking it easy, they'll think I'm lazy. "There remains, then, a Sabbath-rest for the people of God; for anyone who enters God's rest also rests from his own work, just as God did from His. Let us, therefore, make every effort to enter that rest."

(Hebrews 4:9-11)

7. This day/semester/rehearsal/feeling will never end.

"The Lord says, Be strong and courageous. Do not be terrified, for the Lord your God will be with you in all things, wherever you go." (Joshua 1:9)

8. I will never feel good about how my body looks/feels/moves.

"Do you not know that your body is a temple of the Holy Spirit who is in you, whom you have received from God?" (1 Corinthians 6:19) 
9. My instructor/collaborator/fellow dancer makes me crazy.

"Dear friends, let us love one another, for love comes from God. Everyone who loves has been born of God and knows God." (1 John 4:7)

"Be kind and compassionate to one another, forgiving each other, just as in Christ God forgave you." (Ephesians 4:32)

10. This audition will determine my future as a dancer.

"For I know the plans I have for you, declares the Lord. Plans to prosper you and not to harm you; plans to give you hope and a future." (Jeremiah 29:11)

"Trust in the Lord with all your heart, and lean not on your own understanding; in all your ways acknowledge Him and He will make your paths straight." (Proverbs 3:5-6)

11. My dancing is a direct measure of my worth.

"Since you were precious in My sight, you became glorious, and I love you." (Isaiah 43:4)

"You created my inmost being; you knit me together in my mother's womb. I praise You because I am fearfully and wonderfully made; Your works are wonderful, I know that full well." (Psalm 139:13-14)

12. Clearly I am not as good as I thought.

"For I am sure of this, that He who began a good work in you will carry it on to completion until the day of Christ." (Philippians 1:6)

"So God created mankind in His own image; in the image of God He created them."

(Genesis 1:27) 